\title{
Hemo-Vasculogenesis in the Kidney and Heart
}

\author{
Yan $\mathrm{Hu}$ \\ Baotou, China
}

Bachelor of Science, China Agricultural University, 2009

A Dissertation presented to the Graduate Faculty of the University of Virginia in Candidacy for the Degree of

Doctor of Philosophy

Department of Biology

University of Virginia

May, 2016

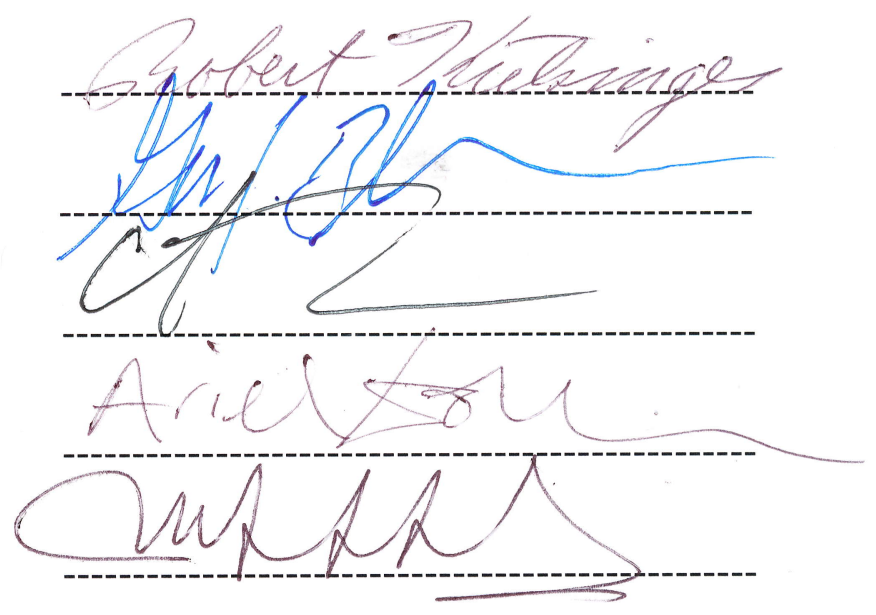




\section{Abstract}

The kidney vasculature plays essential roles in the development and function of the kidney. There is currently very limited information regarding the crucial events that govern the morphogenesis of the renal vasculature, including the origin, lineage relationship and mechanisms involved in its formation. Using inducible transgenic mice that specifically label cells expressing transcription factor stem cell leukemia (SCL), we performed fate-tracing studies combined with colony forming assays (CFCs) and embryonic kidney cross-transplantation studies. We found that the SCL+ precursors in the kidney contribute to the endothelial cells (ECs) and a subpopulation of early hematopoietic precursor cells that form colony forming unit Granulocyte-Erythrocyte-Monocyte-Megakaryocyte (CFU-GEMM) and colony forming unit Granulocyte-Macrophage (CFU-GM) colonies. We also showed that the transplanted embryonic kidneys generate their own vessels and blood cells in situ, which we termed hemo-vasculogenesis. Genetic ablation of these precursors in vivo and cross-transplantation led to the lack of endothelium, demonstrating their vital role during vascular development and organogenesis.

Sphingosine 1-phosphate receptor 1 (S1P1) is one of the five G-protein coupled receptors activated by Sphingosine 1-phosphate, which is crucially involved in the vascular development. However, due to the early lethality of the S1P1 null mice, the role of S1P1 in kidney vascular development has not been determined. To identify the mechanisms governing the development of kidney vasculature, we designed a series of experiments to study the role of S1P1 expressed in the $S C L+$ precursors and their derivatives during kidney development. In this dissertation work, we 
found that the endothelial S1P1 is required for multiple processes during kidney vascular development, including EC proliferation, vSMCs coating, and the development of glomerular capillaries and renal lymphatic vessels. Deletion of S1P1 from the $S C L+$ precursors resulted in endothelial hyperplasia, dilated renal arteries and veins, disrupted vSMC coating of renal arteries and arterioles and the absence of lymphatic endothelium. Remarkably, the knockout mice developed capillary shunts within the glomeruli. These results demonstrated the essential role of S1P1 for the kidney vascular development. Notably, we observed thinner inter-ventricular septum, thinner ventricular myocardial compact layer and dilated myocardial capillaries in hearts of knockout embryos. By cross-transplantation studies of the kidneys from the E12.5 knockout and control mice under the kidney capsule of WT adult mice, we showed that the kidney vascular abnormalities are intrinsic, due to the lack of S1P1 in renal EC precursors, and not secondary to the extra-renal anomalies.

Finally, we found that the $S C L+$ cells contribute to another population of hemovascular precursors in the heart that exclusively differentiate to the endocardium and coronary vascular endothelial cells, including a subset with hemogenic potential. To characterize the contribution of $S C L+$ precursors to the heart, I developed a novel approach to transplant the E9.5 mouse embryonic heart under the kidney capsule of adult host mice, which allows the ex vivo hemo-vasculogenesis and the visualization of beating hearts at the time of harvest. Strikingly, partial ablation of the $S C L+$ precursor from the heart in vivo and cross-transplant model led to the absence of cardiac ECs accompanied with impaired myocardial development, 
which revealed the vital role of $S C L+$ precursors in the development of both cardiac cell types. Furthermore, by timed deletion of S1P1 from the $S C L+$ precursors and their derivatives during early heart development, we uncovered the unique role of endothelial S1P1 in the myocardial and coronary vascular development. The mice with S1P1 deletion showed deficiencies in the myocardial proliferation, reduced ventricular coronary vasculature, and hypertrabeculation.

In summary, these studies have identified an $S C L+$ precursor contributes intrinsically to the hemo-vasculogenesis in both embryonic kidney and heart, which express S1P1 to regulate vascular development and organogenesis. These findings may help advance our understanding of normal vascular development and genetic defects that trigger congenital kidney and heart diseases and development of new treatment for the patients. 


\section{Acknowledgment}

I would like to thank my wonderful mentors, Dr. Maria-Luisa S. Sequeira-Lopez and Dr. Roberto Ariel Gomez, whose dedication, enthusiasm and motivation have made my training an exciting journey. They have spent countless hours on guiding my research, writing, grant applications, trouble shooting and personal issues. Nonetheless, they have kept encouraging me to think outside the box, explore the unknown and defeat the difficulties. It is through their invaluable coaching that I have grown and learned how to be a great scientist.

I would also like to thank all the past and present lab members who have created a warm, supportive and joyful environment during these years. I am thankful to Dr, Ellen Pentz, Dr. Brian Belyea and Dr. Silvia Medrano, who have provided invaluable helps and suggestions on my research, grant applications and manuscript writing. Specially, I would like to thank Dr. Minghong Li, who has taught me many experimental and surgical techniques and been a loving and supportive friend. I would also like to thank Rajwinderjit Kaur, Ted Mehalic, Dani Stumbo, Maria Florencia Martínez and Dr. Masafumi Oka for being great sources of help, friendship, laughter and fun. Last but not least, I would like to thank my fellow graduate students, Dr. Ruth M. Castellanos Rivera and Dr. Eugene E. Lin, who have shared lots of their precious experience on science, career and life with me.

None of this would have been made possible without my thesis committee. Dr. Robert Kretsinger and Dr. George Bloom have been always available for any of my needs. Dr. Christopher Deppmann has been a "life saver" to me, when I had difficulties to find a new committee. Dr. Melissa Henriksen had faithfully served as 
my committee for three years before she left the department. They have provided insightful criticism and thoughts that have strengthened my research.

I am truly grateful to Dr. Jing Yu and Dr. Robert L. Chevalier, Dr. Robert Kretsinger, and Dr. Ellen Pentz, who have supported my applications to the American Heart Association Pre-doctoral Fellowship.

I would also like to thank all the friends in Charlottesville who have made this beautiful town home to me. I would like to give my special thanks to my dear ganma Darien Wei, who has provided unconditional love and fantastic cooking. I am deeply grateful to Darien, Steve Braintwain and many friends in CCCC, who has guided me on my spiritual journey. I would also like to thank Dr. Han Dong for being a great friend and roommate, who have shared the happiness and sadness with me.

Most importantly, I want to thank my mom and dad for everything. Without their love, support, and encouragement, I wouldn't even be here today.

Finally, I want to thank the greatest husband in the world, Fei Yi. Thank you for been so loving, caring and patient. I'm looking forward to starting the next chapter of our life together. 


\section{Table of Contents}

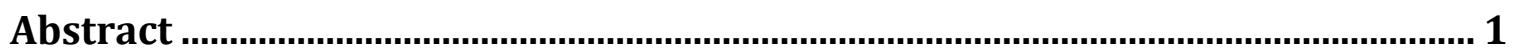

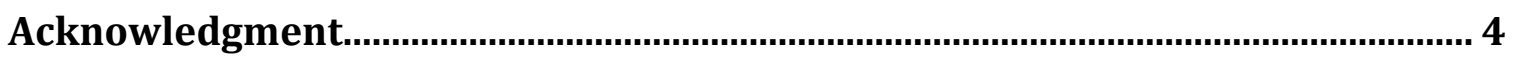

Chapter 1. Introduction....................................................................................... 9

1. Development of the mammalian kidney .............................................................................

1.1 Pronephros, mesonephros and metanephros.................................................................

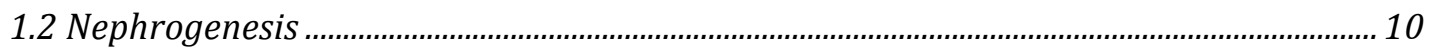

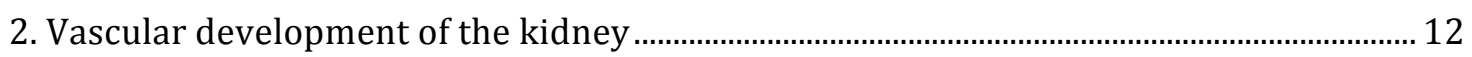

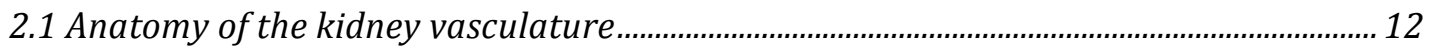

2.2 Development and embryonic origin of renal blood vessels................................................. 14

2.3 Development and embryonic origin of kidney lymphatic vessels...................................... 17

2.4 Regulation of kidney vascular development ................................................................... 18

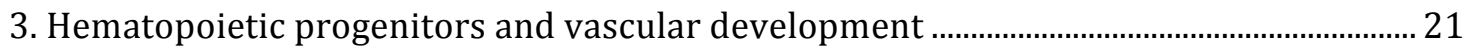

3.1 Hemo-vascular precursors and hemo-vasculogenesis ..................................................... 21

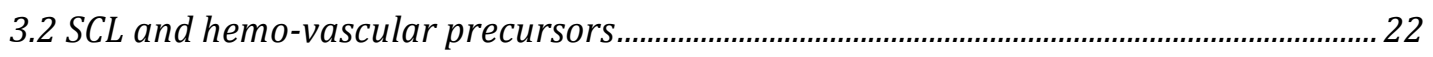

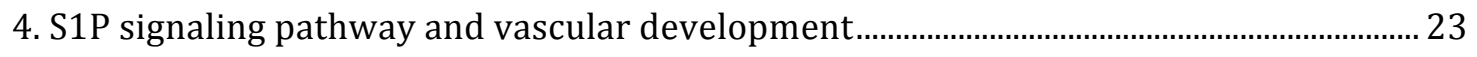

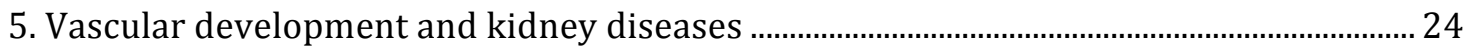

6. Beyond the kidney: hemo-vascular development of the heart ...............................................26

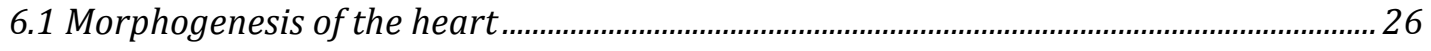

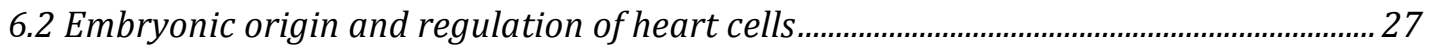

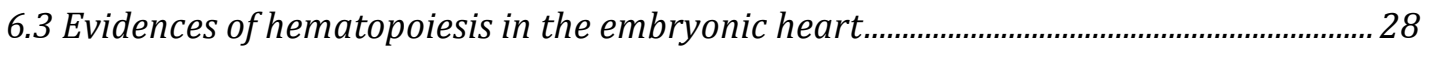

Chapter 2. Hemo-vascular Progenitors in the Kidney Require S1P1 for

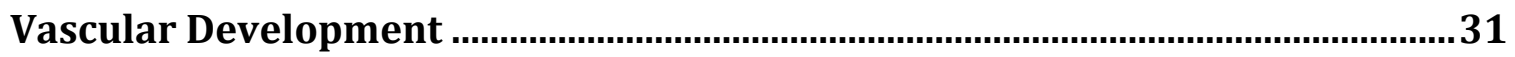

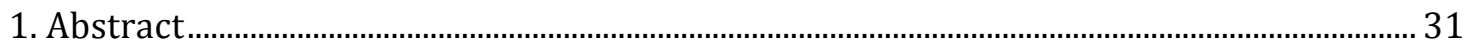




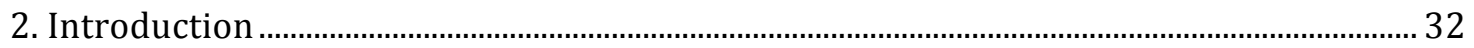

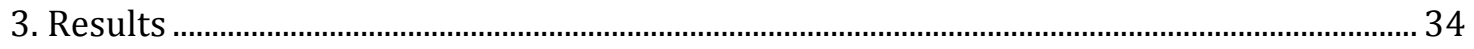

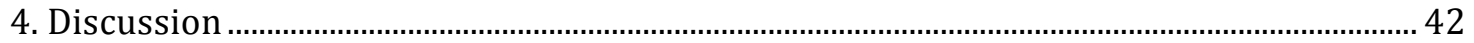

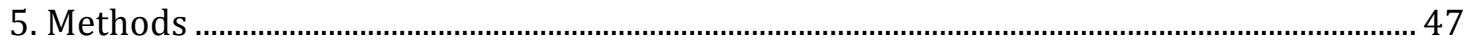

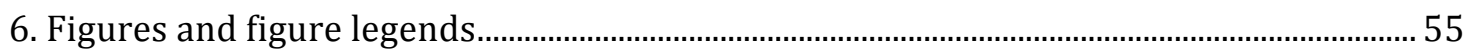

\section{Chapter 3. Endocardium, coronary vascular endothelium and hematopoietic} cells originate from hemo-vascular precursors and regulate heart development through sphingosin-1-phophate receptor 1 ....................................72

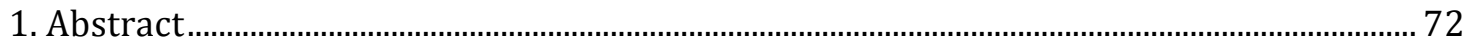

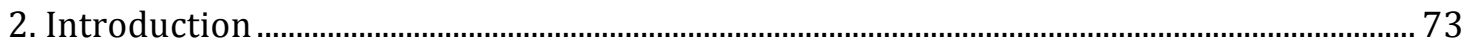

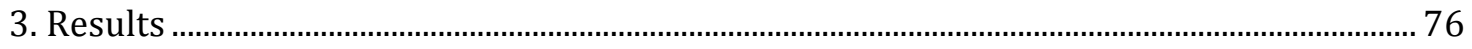

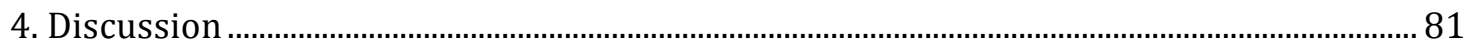

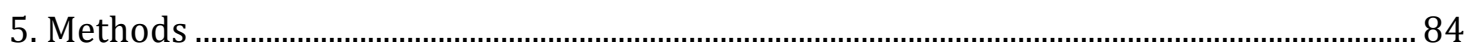

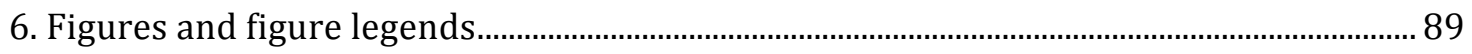

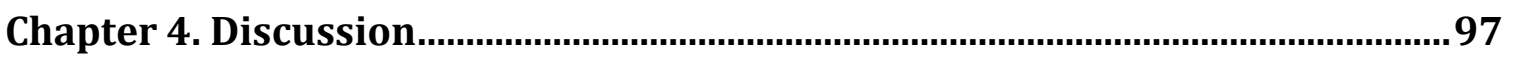

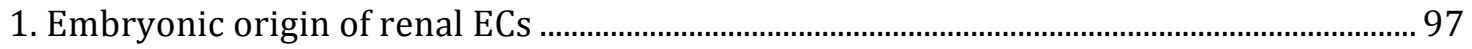

2. Hemo-vasculogenesis in early embryonic kidneys.............................................................. 98

2.1 Initiation of hemo-vasculogenesis in the developing kidney ............................................ 98

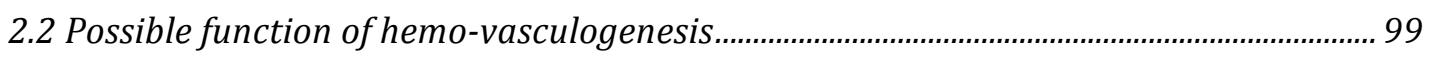

3. Hemo-vasculogenesis in the embryonic heart...................................................................103

4. Role of S1P1 signaling in kidney vascular development .....................................................105

4.1 Possible role of S1P1 in the kidney vascular development................................................105

4.2 Possible role of S1P1 in renal lymphatic development .......................................................109

5. Role of S1P1 in heart development......................................................................................111 
5.1 Possible role of S1P1 in coronary vascular development ..................................................111

5.2 Possible role of S1P1 in myocardium development.............................................................113

6. Conclusions and Future Perspectives ........................................................................................114

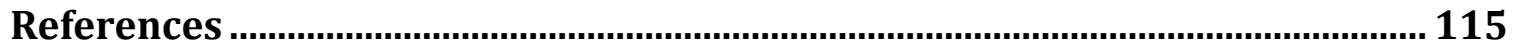




\section{Chapter 1. Introduction}

\section{Development of the mammalian kidney}

The kidneys play essential roles in excretion of metabolic waste products, acid-base control, maintenance of the homeostasis of ions and regulation of blood pressure and extracellular fluid volume and composition. Morphogenesis of the mammalian kidney has been more extensively studied since the1960s. ${ }^{1-5}$ The dissection and detailed characterization of the embryos of human and other mammalian species formed the foundation for our current knowledge of kidney development. In turn, such knowledge is important to understand abnormalities of kidney development and other renal diseases.

\subsection{Pronephros, mesonephros and metanephros}

Before the development of the metanephros, the definitive kidney in mammals, there are two spatially and temporally successive structures that form and regress: the pronephros and the mesonephros. ${ }^{6,7}$ (Figure 1.1) During the third to fourth week of human embryogenesis, the pronephros develops 6-10 pairs of pronephric tubules connecting to the pronephric duct in the neck region (4th-14th somites), which disappears completely by the fourth week of gestation, followed by the development of the mesonephros. ${ }^{6,7}$ Although, pronephros and mesonephros have no major excretory function, their developmental failure results in agenesis of the metanephros and other organs. 6 The mesonephros, a transient excretory organ, contains approximately 40-42 excretory units from the intermediate mesoderm and drains into the mesonephric duct, also called the wolffian duct. ${ }^{6}$ During 4-8 weeks of 
gestation in human, this structure progressively degenerates, although part of the wolffian duct contributes to the male reproductive system. ${ }^{6}$ Beginning with the fifth week of gestation, the caudal part of the wolffian duct gives rise to the ureteric bud of the metanephros, which interacts with and branches into the metanephric mesenchyme, which also derived from the intermediate mesoderm, to form the definitive and functional metanephric kidneys.

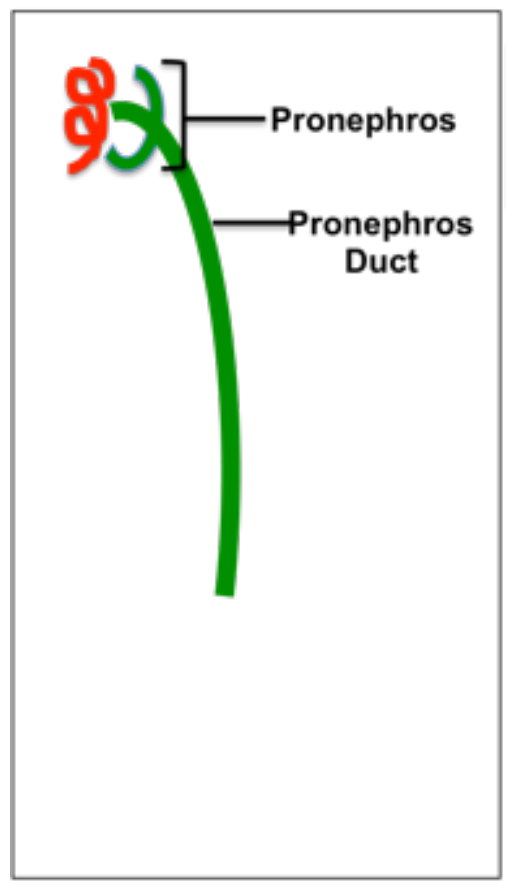

Third week

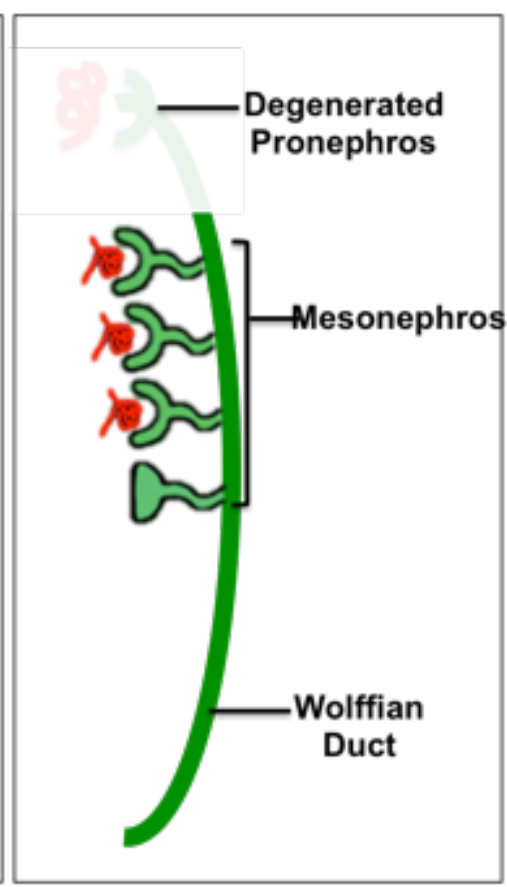

Fourth week

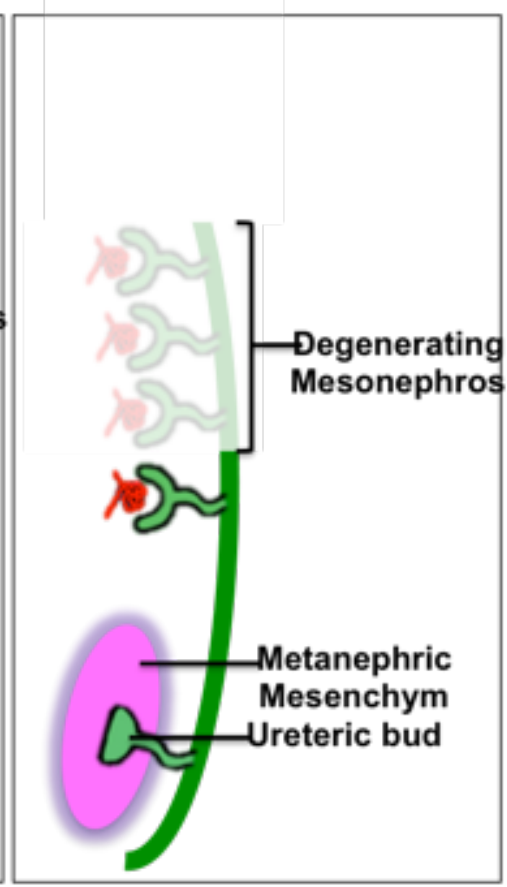

Fifth week

Figure 1.1. Anatomical development of human kidneys. Schematic diagram illustrating the sequential development and degeneration of pronephros, mesonephros and metanephros during human embryogenesis.

\subsection{Nephrogenesis}

The nephron, the basic structural and functional unit of the metanephric kidney, is composed of a glomerulus, the Bowman's capsule and renal tubules. The process of its development is called nephrogenesis. (Figure 1.2) During this process, cells of the metanephric mesenchyme aggregate and condense around each tip of the branching 
ureteric buds to form a cap mesenchyme, which contributes to the epithelial compartment of the future nephrons through a mesenchymal-epithelial transition. A loose mesenchyme surrounding the cap mesenchyme gives rise to the endothelial precursor cells and the stromal precursor cells, which will differentiate into ECs and mural cells, respectively. ${ }^{5}$ After epithelialization, the cap mesenchyme develops into a renal vesicle, which then elongates to form a comma-shaped body and subsequently an S-shaped body. ${ }^{6}$ The proximal segment of the S-shape body will form the distal tubule and fuse to the neighboring ureteric bud, which will differentiate into collecting ducts. Its medial segment will elongate and develop into the loop of Henle and the proximal tubules. Moreover, its distal segment will give rise to the podocytes and Bowman's capsule of the mature glomerulus. ${ }^{7}$ During this process, the lower cleft of the $S$ shape body is invaded by endothelial and mesangial precursors from the loose mesenchyme, which eventually form glomerular capillaries and the mesangium. In humans, nephrogenesis is completed about 34-35 weeks of gestation. In mice and rats, it continues after birth for about 3-7 days, respectively. ${ }^{6}$ 


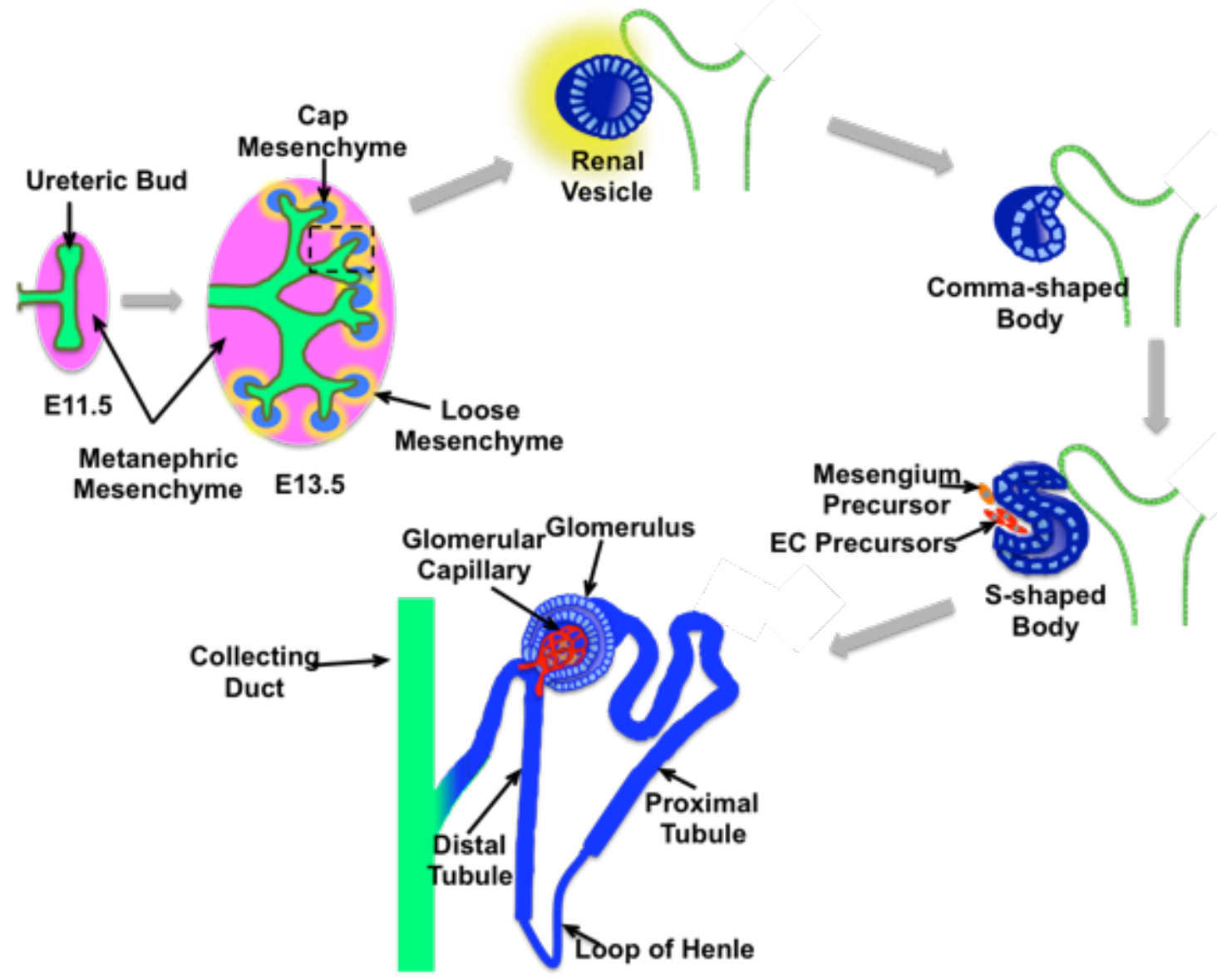

Figure 1.2. Nephrogenesis. Modified from reference ${ }^{8}$ and ${ }^{9}$.

\section{Vascular development of the kidney}

\subsection{Anatomy of the kidney vasculature}

The kidneys are highly vascularized organs that filter approximately $25 \%$ of the volume of the blood that is pumped by the heart daily. The kidney vasculature is composed of arteries, veins, arterioles, capillaries and lymphatic vessels. Blood enters the kidney through renal arteries and passes through the segmental arteries, interlobar arteries, arcuate arteries and interlobular arteries, which further branch to afferent arterioles that connect to the glomerular capillaries. After entering glomeruli, where blood is filtered and cleared of waste products, the blood drains 
into the efferent arterioles and enters the peritubular capillaries and vasa recta, which surround the renal tubules allowing reabsorption of electrolytes and nutrients back to the blood. Then the blood drains into interlobular veins, arcuate veins, and interlobar veins, respectively, and leaves the kidney through renal veins. ${ }^{5}$

(Figure 1.3)

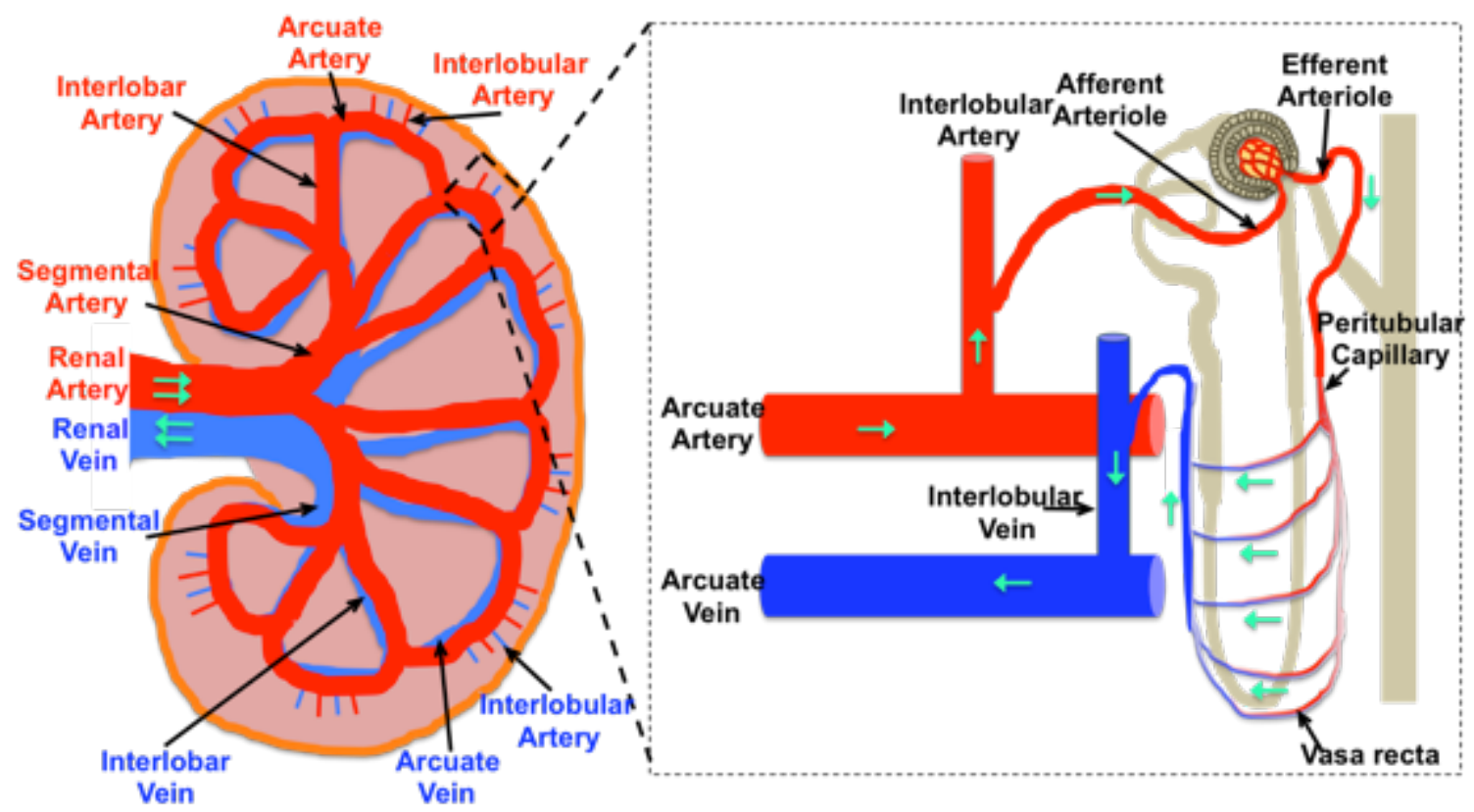

Figure 1.3. The structure of the mammalian kidney vasculature. The diagram on the left shows an overview of the major kidney vessels. The small vessels of the nephron are shown on the right dashed square. Modified from ${ }^{\mathbf{1 0}}$.

Lymphatics are structurally and functionally specialized vessels responsible for maintaining interstitial fluid homeostasis, immune cell trafficking, and absorption of dietary lipids. ${ }^{11}$ The structure of the renal lymphatic vasculature has been described in humans and various animal models.${ }^{12,13}$ In the adult kidney, lymphatic vessels are mainly distributed around the arcuate arteries and interlobular arteries in the cortex. A subset of lymphatic vessels was found within the renal capsule and subcapsular cortex area. (Figure 1.4) ${ }^{5}$ The blind-ended lymphatic capillaries take up 
excess interstitial fluid, proteins and white blood cells and transport them through the larger collecting lymphatic vessels to the veins. In a healthy adult human, the lymphatics drain daily about $8 \mathrm{~L}$ of interstitial fluid containing $20-30 \mathrm{~g} / \mathrm{L}$ of protein back to the blood circulation. RW.ERROR - Unable to find reference:142 Defects in lymphatic development result in accumulated interstitial fluid that causes edema and abnormal immune responses. ${ }^{5}$

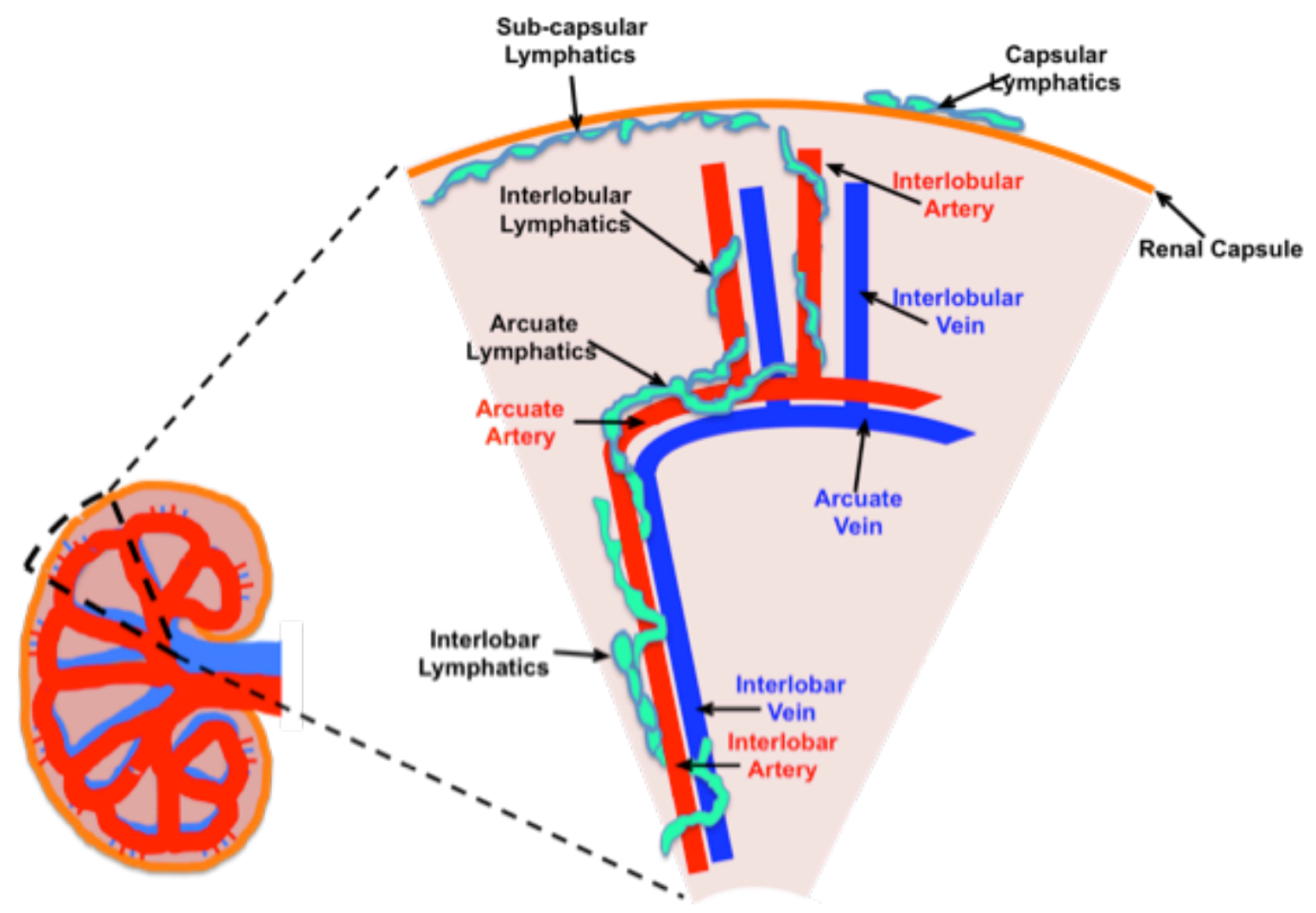

Figure 1.4. Diagram of kidney lymphatic vasculature. From reference ${ }^{\mathbf{5}}$ 2.2 Development and embryonic origin of renal blood vessels

Renal blood vessels are composed of two major layers: endothelial cells (ECs), forming the inner layer of the vessels, and mural cells, including smooth muscle cells (SMCs), renin cells (specialized SMCs that locate in the juxta-glomerular region of the afferent arterioles and express renin) and pericytes. The renal arteries, arterioles and veins are 
wrapped by SMCs whereas the renal capillaries are wrapped by the pericytes. (Figure 1.5) Two distinct processes are involved during renal vascular formation: vasculogenesis and angiogenesis. 9, 14 Vasculogenesis is defined as de novo differentiation and assembly of endothelial tubes followed by recruitment of SMCs that differentiate from local mesenchymal precursors. Angiogenesis is the generation of new vessels from pre-existing ones by proliferation, migration and sprouting of endothelium and recruitment of SMCs. Studies using chimeric mice and inter-species surgical grafts of embryonic kidneys showed that the renal vasculature was generated by a combination of vasculogenesis and angiogenesis. ${ }^{9}$ In the mouse kidney, the first arterioles are formed about 15 days of gestation (E15). For about 10 days after birth, the branching and elongation of arterioles reach to a peak and significantly increase the complexity and surface area of the renal arterial tree. ${ }^{9}$ Note that each tip of the branch needs to connect to a glomerulus to form a functional nephron. Therefore, spatial and temporal control of the differentiation, migration and assembly of the vascular cells are required for the synchronized development of arterioles with nephrons. However, there is currently very limited information regarding the crucial events that govern the morphogenesis of the renal arterial tree. 


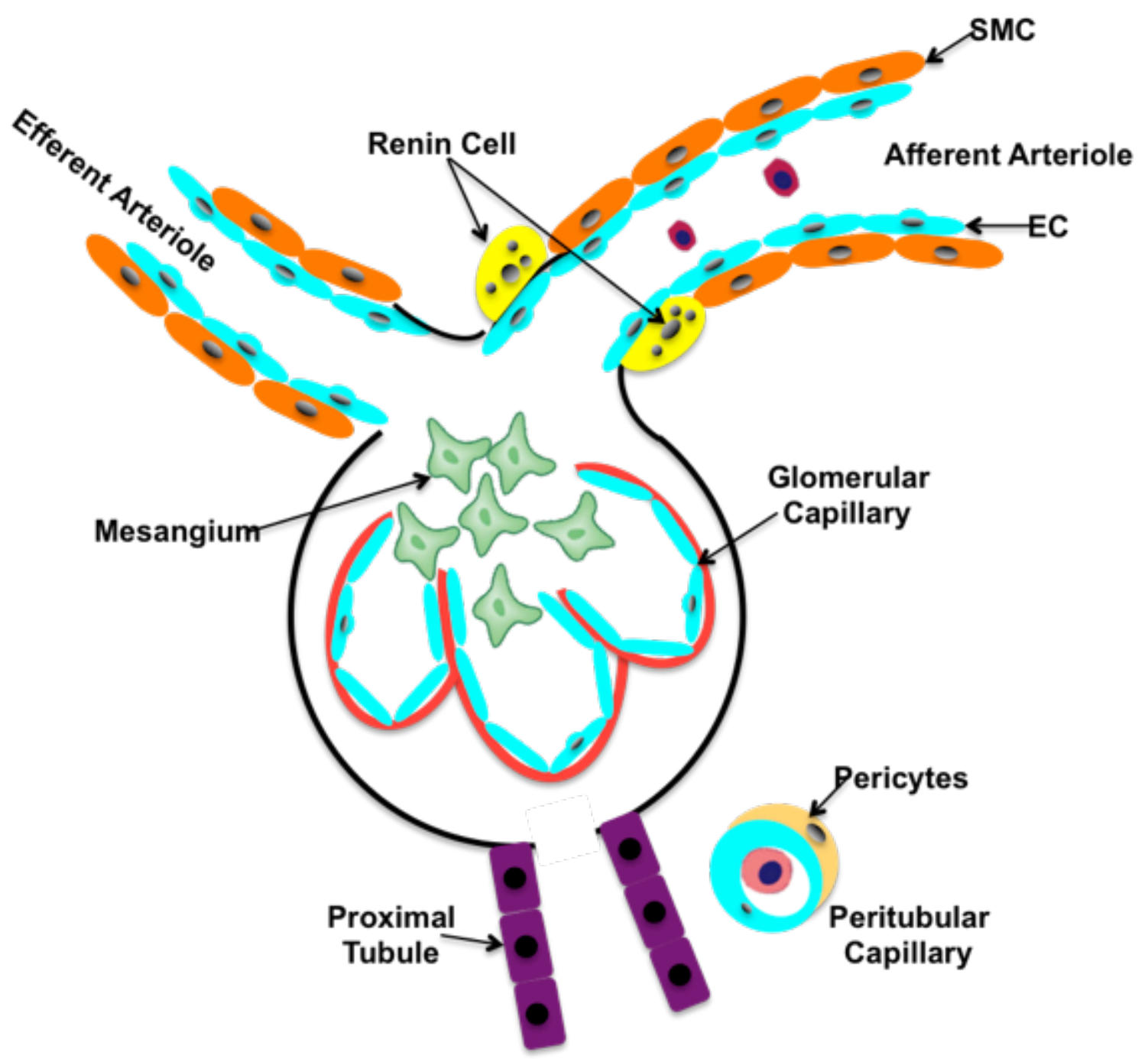

Figure 1.5. Cellular composition of kidney vasculature.

Studies performed by cross-transplantation of mouse pre-vascular embryonic kidneys under the kidney capsule of a host mouse suggested that the progenitors of all the vascular cells were present in the metanephric mesenchyme before the vasculature forms. ${ }^{15}$ Recently, our laboratory identified the earliest metanephric progenitor cell. This progenitor expresses the winged-forkhead transcription factor1 (Foxd1) that give rise to all the mural cells of the renal blood vessels and the glomerular mesangium. ${ }^{16}$ Deletion of Foxd1 and ablation of Foxd1+ precursors using diphtheria toxin A led to 
abnormal renal vascular development, as exemplified by the abnormal orientation of the renal arterial tree, multiple branches of the renal arteries, delayed nephrogenesis and glomerular aneurysms. ${ }^{16,17}$

Despite the discovery of the mural cell precursors, little is known about the cellular origin of the renal ECs. Interestingly, kidney vascular development initiates around E12.5, before it connects to the systemic circulation. Blood cells budding from the endothelium of developing renal vessels were found in cultured E12.5 embryonic kidneys ${ }^{18}$, suggesting that the developing kidney may generate its own vessels and blood cells during early development. In this dissertation, I identified a group of precursors that intrinsically give rise to both vascular endothelium and blood cells in the early embryonic kidney. Deletion of a regulatory molecule in these precursors and ablation of them in mice lead to abnormal renal vascular development. Detailed studies will be discussed in Chapter 2.

\subsection{Development and embryonic origin of kidney lymphatic vessels}

Unlike the blood vessels, lymphatic capillaries are composed of a thin and highly permeable layer of lymphatic endothelium (LEC) that shows distinct morphology and gene expression. 5, 11 Until the last decade, little was known about the development of this system due to lack of specific LEC markers. Using cell culture and microarray analysis, a set of functional marker genes were identified, such as lymphatic vessel endothelial hyaluronan receptor 1 (LYVE-1), vascular growth factor receptor 3 (VEGF-3), podoplanin and prospero-related homeobox gene 1 (Prox-1). 5, 11,19 The histochemical methods using these markers have remarkably 
advanced the knowledge of lymphatic development during normal and pathological states. Notably, none of these markers is exclusively expressed in LECs. For example, LYVE-1 is also expressed in blood vascular ECs during early development. ${ }^{20}$

To date, the cellular origin of LECs is still controversial. A hundred years ago, Florence Sabin proposed that the lymphatic system originate from the veins, which has been further validated later by lineage tracing studies using LECs markers. ${ }^{21,22}$ The formation of lymphatic vessels from veins is termed lymphangiogenesis. In contrast, identification of lymphatic precursors in both embryonic and postnatal life during the last ten years raised a contradicting theory stating that LECs originate independently from lymphangioblasts derived from mesenchyme, and then connect to the veins. ${ }^{23}$ Immunostaining for LYVE-1 and Prox1 in mouse embryos showed that the first lymphatic vessels started to develop in the renal hilum of the E13 kidney and that the renal lymphatic vessels may form by branching from extra-renal lymphatics. ${ }^{13}$

\subsection{Regulation of kidney vascular development}

The major signaling pathways controlling vascular development and stability have been extensively reviewed. ${ }^{5,9}$ In this section, the details of several factors that may connect to this dissertation study, including vascular endothelial growth factor (VEGF), angiopoietins, platelet-derived growth factor (PDGF), transforming growth factor beta (TGF- $\beta$ ), hypoxia and hemodynamic forces are summarized below. The S1P signaling pathway will be discussed in more detail in section 4 . 
The VEGFs are a family of heparin-binding growth factors consisting of five members: VEGF-A to -D and placental growth factor. They bind to three main receptor tyrosine kinases, VEGFR-1/Flt-1, VEGFR-2/Flk-1 and VEGFR-3/Flt4 and co-receptors, such as neuropilins. VEGF-A is the core regulator of differentiation, proliferation, survival and permeability of the vascular ECs during development and adult life. Even knockout of one allele of VEGF-A in mice is lethal. Alternative splicing produces several soluble and matrix-bound VEGF-A isoforms that function distinctively during angiogenesis. ${ }^{24-26}$ In the kidney, VEGF-A is highly expressed in podocytes, starting in the S-shaped body, whereas weak expression was found in distal tubules. ${ }^{26,27}$ VEGFR-2/Flk-1, expressed in progenitors of ECs responds to the dose gradient of VEGF-A produced by podocytes and guides formation of glomerular capillary loops. Over expression or deletion of VEGF-A in podocytes leads to glomerular developmental defects. ${ }^{26}$ VEGF-C, binding to VEGFR-2 and VEGFR-3, regulates blood vascular formation during early embryogenesis and later lymphangiogenesis. ${ }^{5,24}$

Angiopoietin and Tie signaling system consists of four secreted glycoproteins, angiopoietin1-4 (Ang-1-4), and two endothelial tyrosine receptor kinases, Tie-1 and Tie-2. ${ }^{25,} 28$ Ang-1 expression is widely distributed in the metanephric mesenchyme, tubules and podocytes, whereas Ang-2 expression is transient in SMCs, mesangium and tubules close to adult vasa recta. Gene knockout studies in mice showed that the Ang-1 induced Tie- 2 signaling, which is suppressed by Ang- 2 and Tie- 1 is crucial for recruitment of mural cells and renal endothelial differentiation and survival. Furthermore, the Angiopoietin signaling is regulated by VEGF-A and integrin. ${ }^{28}$ 
The PDGF system consists of four isoforms (PDGF-A to -D) and two receptor chains, PDGFR- $\alpha$ and PDGFR- $\beta$. PDGF-A and PDGF-B are released as homodimers or heterodimers (AA, $\mathrm{AB}$ and $\mathrm{BB})$, whereas PDGF-C and PDGF-D only form homodimers. ${ }^{5,29}$ During kidney development, PDGF-B expressed by ECs reacts with PDGFR- $\beta$ in mural cells and mesangium, regulating vascular coating and glomerular development, respectively. Loss of PDGF-B or PDGFR- $\beta$ leads to vascular leakage and glomerular aneurysms. ${ }^{29}$

The TGF- $\beta$ superfamily is another essential regulator during vascular development. The TGF- $\beta$ s and their receptors are expressed in ECs and mural cells, controlling the interplay between both cells types. The canonical signaling cascade is composed of ligand TGF- $\beta$ (TGFB-1 to -3), type I and type II serine/threonine kinase receptors and intracellular Smad transcription factors. During vascular development, mural cells proliferate and differentiate in response to TGF- $\beta$ signaling and are recruited to the nascent endothelial vessels by PDGF-B. ${ }^{5,30}$

During embryogenesis, when passive diffusion no longer provides sufficient $\mathrm{O}_{2}$ and nutrients to cells, hypoxia induces expression of heterodimeric transcription factor hypoxia inducible factors (HIFs). The HIFs (HIF-1 $\alpha$, HIF-2 $\alpha$ and HIF-1 $\beta$ ) activate many genes involved in vascular development, cellular survival, erythropoiesis and metabolism, including VEGF and PDGF-B. Mice lacking HIFs die in utero as a result of vascular defects, which can be rescued by adding VEGF in vitro. ${ }^{31}$ Histochemical studies and in vitro kidney culture under hypoxic conditions suggested that HIF-1 $\alpha$, HIF- $2 \alpha$ and HIF-1 $\beta$ induced by hypoxia were highly expressed in podocytes and microvascular ECs, co-localized with VEGF expression. ${ }^{32}$ 
Hemodynamic forces generated by blood flow were first identified as a regulator of vascular development in the yolk sac. ${ }^{33}$ In vitro analysis of cultured ECs showed that shear stress generated by steady laminar flow activated a mechanosensory complex, comprised of Flk-1, vascular endothelial cell cadherin (VE-cadherin) and platelet endothelial cell adhesion molecule 1 (PECAM-1), which changed the morphology and polarity of the ECs to align parallel to the direction of flow. ${ }^{34}$ However, these molecules were thought to regulate adult vessels. ${ }^{35}$ In vivo studies using gene deficient mice suggested that different mechanosensitive components are involved during embryonic vascular development, including several G-protein coupled receptors, nitric oxide release molecules and calcium channels that are controlled by primary cilia of ECs. ${ }^{34,35}$

\section{Hematopoietic progenitors and vascular development}

\subsection{Hemo-vascular precursors and hemo-vasculogenesis}

About a century ago, based on Florence Sabin's observations in cultured chick blastoderms, Murray proposed the concept of the hemangioblast. 22, 36 Hemangioblasts were defined as mesodermal progenitor cells giving rise to both ECs and blood cells, which were initially called angioblasts (progenitors of ECs) by Sabin. ${ }^{22}$ This hypothesis is now commonly accepted and supported by in vitro studies in human and mouse ES cell cultures and in vivo studies in multiple model organisms. Genetic analysis on ES cells and zebra fish embryos suggest that several genes are essential for the fate specification of hemangioblasts from Brachyury+ 
mesoderm, including Flk1, stem cell leukemia (SCL), and Runt-related transcription factor 1 (Runx1), which have been nicely reviewed. ${ }^{37}$

During mouse embryogenesis, hematopoiesis (generation of blood cells) occurs in multiple sites. Two major distinct waves have been described. The first hematopoietic wave (primitive hematopoiesis) takes place in the yolk sac at embryonic day 7.5 (E7.5), where nucleated erythroblasts and megakaryocytes are generated. These progenitors enter the circulation at E8.5 and home to the fetal liver as it starts to form. ${ }^{38}$ Definitive hematopoiesis is established within the embryo proper in the para-aortic splanchnopleura (P-Sp)/aorta-gonadmesonephros (AGM) region at E9.5 and generates hematopoietic stem cells (HSCs) with a long-term repopulating activity, which give rise to all hematopoietic lineages. ${ }^{39}$ Recently, hematopoiesis has been discovered in the mouse embryonic head and heart, expanding the areas with hemogenic potential in the embryo. ${ }^{40-42}$ Studies using chick-quail chimera assays demonstrated that the splanchnopleuric mesoderm-derived ECs in the ventral aorta give rise to HSCs, and they were named hemogenic endothelium (hemogenic EC). These progenitors were later identified in the yolk sac, dorsal aorta, fetal liver and fetal bone marrow of mice and human embryos. ${ }^{37}$ The lineage relationship between hemangioblast and hemogenic ECs is unknown due to lack of specific markers.

\subsection{SCL and hemo-vascular precursors}

The helix-loop-helix (bHLH) transcription factor stem cell leukemia (SCL/Tal1), one of the first markers identified in hemangioblasts, is essential for specification of 
hemangioblasts and their differentiation to hemogenic ECs and all hematopoietic lineages. ${ }^{43-46}$ Two distinct elements within the $S C L$ locus guide its expression to the endothelial and hematopoietic compartments, respectively. An SCL 5' endothelial enhancer directs SCL expression in the endothelium, whereas an SCL 3' enhancer is responsible for SCL expression in early hematopoietic progenitors. ${ }^{44}$ SCL null mice develop anemia and die by E9.5 to E10.5. ${ }^{47,48}$ In embryonic kidneys of human and mouse, $S C L+$ cells were identified in the developing mesenchyme and vascular endothelium. ${ }^{49}$ Similarly, using an SCL-LacZ mice with a LacZ reporter gene knocked into the $S C L$ locus, another group showed that $S C L$ expression is detected in the endocardium as early as E8.5. ${ }^{50}$ The fate of $S C L+$ cells in the embryonic kidney and heart has not been explored previously.

\section{S1P signaling pathway and vascular development}

Sphingosine 1-phosphate (S1P) is a bioactive sphingolipid crucial in many biological processes, including angiogenesis. ${ }^{51}$ It activates a family of five G-protein coupled receptors (S1P1-S1P5). The variety of responses mediated by S1P receptors depends on the type of receptor and its coupled downstream effectors expressed in a given cell. ${ }^{52}$ The S1P1 receptor is highly expressed in precursors of the kidney arterioles. Generation of mice with LacZ knockin into the S1P1 locus showed that the receptor is not only expressed in ECs but also in vascular SMCs. ${ }^{52}$ It has been reported that S1P1 functions autonomously in ECs since the phenotype observed in S1P1fl/fl;Tie2 Cre mice mimics the one of the S1P1 null mice. ${ }^{53}$ S1P1 null mice seem to develop normally until E11.5, but at E12.5-E13.5, the embryos develop severe edema and hemorrhages and die in utero by E14.5, possibly due to the failure of 
migration and/or differentiation of vascular SMCs and pericytes, demonstrating the critical role of S1P1 in vascular maturation. ${ }^{54}$ Recent studies show that S1P1 inhibits sprouting angiogenesis of retinal vessels in mice by regulating interactions between VE-Cadherin and VEGFR2 during vascular development. ${ }^{55,56}$ Other studies showed that hemodynamic forces and circulating S1P generated by erythrocytes activate S1P1, respectively, to stabilize blood vessels during development. ${ }^{57,58}$ Because the aforementioned knockout mice die before the kidney develops its own vasculature, the role of S1P1 in renal vascular development is unknown.

In the heart, S1P1, S1P2 and S1P3 are widely expressed in all cardiac cell types and are involved in contractility, hypertrophy and cardioprotection. ${ }^{59}$ S1P1 is predominantly expressed in both cardiomyocytes and ECs. During mouse embryogenesis, S1P1 expression was first identified in the E8.5-E9.5 heart and later in the developing vascular system. ${ }^{60}$ Previous studies using the S1P1 specific agonist SEW2871 showed that the S1P1 receptor couples exclusively to the $\mathrm{G}_{\mathrm{i}}$ protein to inhibit cAMP and antagonize cardiomyocytes contractility. ${ }^{61}$ However, the role of S1P1 in cardiac ECs during heart development is unknown.

\section{Vascular development and kidney diseases}

Kidney diseases can be divided into two major categories: acute kidney injury (AKI) and chronic kidney disease (CKD). AKI is a rapid loss of kidney function within days, which could be caused by renal ischemia and reperfusion injury (IRI), inflammation, urinary tract obstruction and exposure to harmful substances. On the other hand, CKD is a gradual loss of kidney function over time. The main causes of CKD are 
diabetes, hypertension, glomerulonephritis (inflammation and damage of glomeruli), malformation and inheritable polycystic kidney disease. Both AKI and CKD, especially at the end-stages, have shown an alarming increase for the past 30 years in the US and worldwide. ${ }^{62,63}$ Many kidney diseases have primary or secondary vascular lesions, which can be accelerated by risk factors, such as hypertension, diabetes and obesity. 63

Generation of new vessels in the kidney is not confined to embryonic development but is also a mechanism activated in response to disease. ${ }^{63}$ To compensate for the renal vascular rarefaction that results from diseases and consequent loss of blood flow and oxygen, neovascularization is triggered, resulting in a formation of new capillaries from preexisting vessels or recruitment of endothelial progenitors cells (EPCs). Transplantation studies have shown that EPCs from bone marrow improved the vascular regeneration in injured and ischemic kidneys and other tissues. In addition, the presence of stromal progenitor cells within the kidney capsule, cortex and papilla, which migrate to the sites of renal injury, has been demonstrated. ${ }^{64}$ The functions of the intrarenal and extrarenal progenitor cells during tissue repair and regeneration remain to be studied.

However, studies in CKD models suggested that the newly formed vessels might display abnormal architecture and/or impaired function, which may exacerbate disease progression. 63, 65, 66 Therefore, factors controlling remodeling and stabilization of a new vascular network during disease is critical for the repair of kidney damage. A recent study showed that S1P1 expressed in ECs had protective 
roles against IRI by regulating EC barrier integrity and endothelial HSP27 expression. 67

Thus, defining the mechanisms that regulate differentiation and assembly of vascular progenitor cells could lead to the discovery of new treatments for congenital and acquired vascular diseases including those related to the kidney and the systemic circulation.

\section{Beyond the kidney: hemo-vascular development of the heart}

CKD is closely associated with cardiovascular diseases (CVD). According to a report from the American Heart Association, CVD is the leading cause of death in patients with end-stage CKD and those treated with hemodialysis and transplantation. In turn, patients with heart diseases have an elevated risk of developing CKD. The frequent co-existence of CKD and CVD may be caused by common risk factors, such as hypertension, diabetes, obesity and aging. ${ }^{68}$ The regulatory networks controlling co-development of both diseases are not fully understood. Similar to kidney diseases, many genes involved in cardiovascular development are re-expressed during CVD, which contributes to the progression of heart failure. 69 Hence, elucidating the common mechanism underlining vascular development in both organs may open the window for new treatment of kidney and heart diseases.

\subsection{Morphogenesis of the heart}

The heart is one of the first organs to develop and function during mammalian development. The human heart develops on day 18-19 post-fertilization, which in mice is equivalent to embryonic day 7.5 (E7.5). Taking the mouse as an example, 
morphogenesis of the heart begins with a flat sheet of mesodermal heart progenitor cells, called cardiac crescent, lining across the midline and lateral to the neural plate. By E8.5, the cardiac crescent fuses at the midline to form the primary linear heart tube and starts to beat. In the mean time, a population of second heart field (SHF) progenitor cells appear close to the anterior and dorsal area of the heart tube, which later migrate to the heart tube and contribute to its elongation and right looping at E9.5. The looping process forms primitive ventricles, atria and the outflow tract. From E10.5 to E15, the heart forms four well-defined chambers, followed by formation of the valves and septum. ${ }^{70}$ (Figure 1.6)
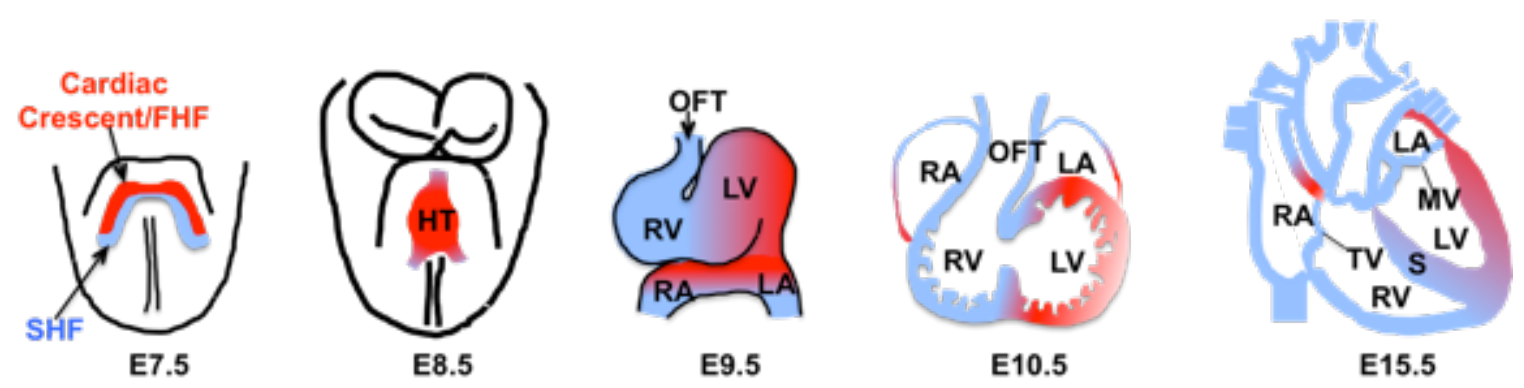

Figure 1.6. Development of the mouse heart. ${ }^{71}$ FHF: first heart field (in red); SHF: second heart field (in blue); HT: heart tube; OFT: out-flow tract; RV: right ventricle; LV: left ventricle; RA: right atria; LA: left atria; S: septum; TV: tricuspid valve; MV: mitral valve.

\subsection{Embryonic origin and regulation of heart cells}

The cellular constituents of the adult heart include ECs, cardiomyocytes, smooth muscle cells (SMC) and cardiac fibroblasts. The endothelium forms the endocardium (the inner lining of the heart chambers), the inner layer of the coronary vessels and the inner lining of cardiac valves. Cardiomyocytes are the contractile muscle cells forming the myocardial wall of the heart chambers. SMCs contribute to the coronary vessels and inflow aorta and outflow aorta. At last, cardiac fibroblasts are widely 
distributed connective tissue cells that are intimately associated with ECs and cardiomyocytes. ${ }^{72}$ Lineage tracing studies in chick and mice demonstrated that two genetically distinct populations of multipotent progenitors give rise to the heart cells. ${ }^{69}$ The first heart field (FHF) progenitors located in the cardiac crescent give rise to endocardium and contractile myocardium of the primary linear heart tube and later contribute to the left ventricle and part of both atria. During looping of the heart tube, the multi-potent SHF progenitors marked by the Isl1 expression, migrate to the heart tube and later differentiate to ECs, SMCs and cardiomyocytes of the right ventricle, part of both atria and the outflow tract. ${ }^{73}$ Based on mutant mice studies, many transcription factors were identified during morphogenesis of heart. The major regulatory genes expressed in FHF and SHF progenitors are listed in Table 1. 74,75 Due to the heterogeneous origin of the heart cells and lack of specific cell type markers, our understanding of the precise regulation of cell fate decision during heart development has been hampered. A recent work identified the lineagecommitted cardiomyoblasts marked and regulated by the HOP homeobox (Hopx) gene. Specification of the cardiac endothelium remains largely unknown. ${ }^{76,77}$

\subsection{Evidences of hematopoiesis in the embryonic heart}

Since the 1980s, several groups have reported hematopoiesis in the mammalian fetal heart. First, "blood islands" (primitive erythroblasts surrounded by ECs), presumably developing coronary vessels, were identified in the apical incisures of human embryonic hearts. ${ }^{78}$ Further, immunohistochemistry in mouse embryonic hearts showed similar structures in the sub-epicardium and endothelium of outflow tract cushions in the E11-14 mouse heart. ${ }^{42}$ Interestingly, another group has 
demonstrated the existence of hemogenic endocardium in early E8-E9.5 mouse hearts, which expresses and requires Nkx2.5 and Isl1 for their differentiation. Together, these data suggest a transient hemogenic function of the early embryonic heart. However, the embryonic origin and function of these cardiac hemogenic progenitors and their lineage relationship with known cardiac cells remains to be investigated.

In summary, identifying the early vascular progenitors in the kidney and cardiovascular system and understanding the molecular mechanisms underlying their differentiation and assembly may provide fundamental new knowledge with potential benefit for children and adults affected by congenital and acquired diseases of the kidneys, heart and blood vessels. 
Table 1.1 The major regulatory genes expressed in FHF and SHF progenitors. ${ }^{74,75}$

\begin{tabular}{|c|c|c|c|c|}
\hline Gene & $\begin{array}{c}\text { FHF } \\
\text { expressio } \\
n\end{array}$ & $\begin{array}{c}\text { SHF } \\
\text { expressio } \\
n\end{array}$ & Functions in heart development & $\begin{array}{l}\text { Referenc } \\
\text { e }\end{array}$ \\
\hline $\begin{array}{c}N k \times 2 \\
5\end{array}$ & Yes & Yes & $\begin{array}{l}\text { Regulating myocardial } \\
\text { differentiation; Restricting } \\
\text { progenitor proliferation }\end{array}$ & 79,80 \\
\hline $\begin{array}{c}\text { GATA } \\
4 \\
\end{array}$ & Yes & Yes & $\begin{array}{l}\text { Regulating the onset of cardiac } \\
\text { differentiation }\end{array}$ & 81 \\
\hline Hand1 & Yes & No & $\begin{array}{l}\text { Regulating formation of the left } \\
\text { ventricle }\end{array}$ & 82 \\
\hline Hand2 & Yes & Yes & $\begin{array}{l}\text { Regulating formation of the right } \\
\text { ventricle }\end{array}$ & 83 \\
\hline Tbx 5 & Yes & No & $\begin{array}{l}\text { Regulating growth and } \\
\text { differentiation of progenitors of left } \\
\text { ventricle and atria by controlling } \\
\text { other transcript factors like GATA4. }\end{array}$ & 84 \\
\hline$T b \times 1$ & Yes & Yes & $\begin{array}{l}\text { Regulating proliferation in the } \\
\text { second heart field by activating } \\
\text { Fgf10 }\end{array}$ & 85,86 \\
\hline Fgfo & No & Yes & $\begin{array}{l}\text { Regulating proliferation of SHF cells } \\
\text { and neural crest cells }\end{array}$ & 87,88 \\
\hline $\operatorname{Mef} 2 c$ & Yes & Yes & $\begin{array}{l}\text { Required for SHF development to the } \\
\text { venous and arterial pole }\end{array}$ & 89 \\
\hline$T b \times 20$ & Yes & Yes & $\begin{array}{l}\text { Dose-dependently regulating } \\
\text { development of both FHF and SHF } \\
\text { progenitors }\end{array}$ & 90 \\
\hline Isl1 & No & Yes & $\begin{array}{l}\text { Regulating migration, proliferation, } \\
\text { survival and fate specification of SHF } \\
\text { progenitors }\end{array}$ & 73,90 \\
\hline Foxh1 & No & Yes & $\begin{array}{l}\text { Essential for right ventricle } \\
\text { development and partially required } \\
\text { for outflow tract development }\end{array}$ & 91 \\
\hline
\end{tabular}




\section{Chapter 2. Hemo-vascular Progenitors in the Kidney Require S1P1 for Vascular Development}

Formatted as a first-authored manuscript and published as:

Hemo-vascular progenitors in the kidney require S1P1 for vascular development. Hu Y, Li MH, Göthert JR, Gomez RA, Sequeira-Lopez ML. J. Am. Soc. Nephrol 2015 Nov doi: 10.1681/ASN.2015060610

\section{Abstract}

The close relationship between endothelial and hematopoietic precursors during early development of the vascular system suggested the possibility of a common, yet elusive precursor, for both cell types. Whether similar or related progenitors for endothelial and hematopoietic cells are present during organogenesis is unclear. Using inducible transgenic mice that specifically label endothelial and hematopoietic precursors we performed fate-tracing studies combined with colony forming assays and cross-transplantation studies. We identified a progenitor, marked by the expression of the helix-loop-helix transcription factor stem cell leukemia (SCL/Tal1). During organogenesis of the kidney, $S C L+$ progenitors give rise to endothelium and blood precursors with multipotential colony forming capacity, indicating that the $S C L+$ cells constitute the long sought-after precursor for hemovasculogenesis, the concomitant formation of blood and vessels. Further, these progenitors require Sphingosine 1-phosphate via its S1P1 G-protein coupled receptor for the appropriate morphogenesis of the kidney vasculature including glomerular capillary development, arterial mural cell coating and lymphatic vessel development. Overall, these studies show that $S C L+$ progenitors with hemogenic 
capacity originate and differentiate within the early embryonic kidney by hemovasculogenesis, and underscore the importance of the S1P pathway in vascular development.

\section{Introduction}

A common progenitor for blood and vessels, the hemangioblast, was proposed about 100 years ago. ${ }^{92}$ Recent studies provided evidence supporting the existence of hemangioblasts and identified transcription factors involved in their differentiation. 93-97 However, their presence and contribution in vivo to the development of blood and vessels is still controversial. ${ }^{98}$ Further, the contribution of such progenitor to blood/blood vessel formation during early organogenesis remains to be tested. The helix-loop-helix transcription factor stem cell leukemia (SCL/Tal1) not only identifies putative hemangioblasts but also is essential for their differentiation. ${ }^{43,44}$ Within the SCL locus, a 5' enhancer directs its expression to the endothelium and a 3' enhancer guides expression to early hematopoietic precursors. ${ }^{44}$ Using mice with tamoxifen inducible cre expression driven by either enhancer, 99,100 we designed experiments to trace the fate of $S C L+$ progenitors and define their role in hemovasculogenesis in the embryonic kidney.

Interestingly, kidney vascular development initiates, in mice, around embryonic day (E)12.5, before it connects to the systemic circulation. Based on embryonic kidney cross-transplantation studies and single cell micro-aspiration followed by RT-PCR, we suggested that the embryonic kidney possesses all the necessary precursors for the development of the kidney vasculature. 101 In addition, we observed hematopoietic cells budding from the endothelium in embryonic mouse kidneys 
grown in culture, ${ }^{18}$ suggesting that the developing kidney generates its own vessels and blood during early development. Although we recently identified the earliest precursor for the mural cell of the kidney arterioles (i.e. smooth muscle cells [SMCs]), ${ }^{16,17}$ the origin of the renal endothelial cells (ECs) is still not clear.

S1P is a bioactive sphingolipid crucial in many biological processes, including angiogenesis. ${ }^{51}$ It activates a family of five G-protein coupled receptors. The variety of responses mediated by S1P receptors depend on the receptor type and their coupled downstream effectors expressed in a given cell. The S1P1 receptor is highly expressed in precursors of the kidney arterioles and later in ECs and vascular SMCs. 52 It has been reported that S1P1 functions autonomously in ECs because the phenotype observed in S1P1/fff; Tie2 ${ }^{\text {Cre }}$ mice mimics the one found in S1P1 knockout (S1P1KO) mice. ${ }^{53}$ S1P1KO mice seem to develop normally until E11.5, but at E12.5E13.5 they develop severe edema and hemorrhages and die in utero by E14.5, presumably due to a role of S1P1 in vascular maturation. ${ }^{54}$ Because the aforementioned knockout mice die before the kidney develops its vasculature, the role of S1P1 in renal vascular development is unknown.

We therefore designed a series of experiments to define whether 1) the prevascular embryonic kidney possesses hemo-vascular precursors, 2) $S C L+$ precursors give rise to vascular endothelium and hematopoietic progenitor cells, 3) hemovasculogenesis occurs in the embryonic kidney and 4) S1P-S1P1 signaling controls the development of the kidney vasculature. 


\section{Results}

\subsection{SCL+ Precursors Give Rise to Renal Vascular Endothelial Cells}

To label the EC-SCL and HSC-SCL precursors generated during definitive hematopoiesis which begins around E10.5 in mice, ${ }^{102}$ we performed daily maternal tamoxifen injections from E9.5 to the day before harvesting EC-SCL-Cre-ERT+/;R26 ${ }^{\text {LacZ/+ }}$ and HSC-SCL-Cre-ERT $T^{+/ ; R 26^{\text {LacZ/+ }}}$ embryos. As shown in Figure 1, EC-SCLCre-ERT+/;R26 LacZ/+ embryos $\beta$-gal+ cells from the EC-SCL lineage were widely spread within the metanephric mesenchyme around the dividing ureteric bud (at E12.5) and formed nascent vascular endothelial tubes with hemoglobin $+(\mathrm{Hb}+)$ erythroblasts in their lumen (at E13.5). By E15.5, the kidney has developed glomerular and peritubular capillaries, arterioles with SMC coating and veins. ECs of all types of blood vessels were $\beta$-gal+ in kidneys of $E C$-SCL-Cre-ERT $T^{+/ ;} R 26^{L a c Z /+}$ mice at E15.5 and E17.5, suggesting their EC-SCL origin (Figure 2.1A). Interestingly, kidneys from HSC-SCL-Cre-ERT ${ }^{+/ ;}$;R26 $6^{\mathrm{LacZ} /+}$ mice (at E12.5 to E17.5), showed a small population of vascular ECs in addition to the expected putative blood cells derived from the HSC-SCL precursors (Figure 2.2A). Further, co-staining by immunofluorescence showed that EC-SCL and HSC-SCL lineage cells expressed the EC marker PECAM-1 (CD31) but did not express $\alpha$-smooth muscle actin ( $\alpha$-SMA), myosin heavy chain (MHC) or the lymphatic endothelial marker LYVE-1 (Figure 2.1B, Figure 2.2B), suggesting that kidney ECs have a distinct origin from the mural cells of arteries and veins and from the lymphatic endothelium. In addition to the early metanephric mesenchyme and typical embryonic hematopoietic sites (yolk sac, aorta-gonad-mesonephros and fetal liver), HSC-SCL lineage cells were observed 
in multiple developing organs and structures, such as heart, brain, lung, gut and limbs (not shown).

To trace the fate of EC-SCL and HSC-SCL precursors generated before E9.5, we injected tamoxifen at E6.5 to allow the Cre-LacZ expression span from E6.5 to E8.5, encompassing the period of yolk sac hematopoiesis (E7.5). In E13.5 embryos, $\beta$-gal+ cells derived from both precursors were present in the kidney (Figure 2.1, C-D). Similar to the EC-SCL-Cre-ERT $+/ ; R 26^{L a c Z /+}$ mice injected at E9.5, the labeled EC-SCL derived cells were positive for PECAM-1 (Figure 2.1C). However, in the kidney of HSC-SCL-Cre-ERT+/;R26 LacZ/+ mice, cells co-expressing $\beta$-gal and SCL were mainly distributed in the undeveloped mesenchyme and did not overlap with Flk1+ endothelium (Figure 2.1D). It is possible that these cells are from early progenitors before the commitment to the EC lineage.

3.2 SCL+ Hematopoietic and Hemogenic Endothelial Progenitors In the Early Embryonic Kidney

To investigate in vivo the presence of erythroid progenitors in the early embryonic kidney, we crossed ER-GFP-Cre mice (harboring a GFPcre knockin into the erythropoietin receptor locus) to R26R-LacZ mice. Figure 2 shows that in the E13.5 kidney, $\beta$-gal+ erythroid cells are also positive for hemoglobin $(\mathrm{Hb})$, Nanog and hematopoietic precursor markers, such as Runx1 and SCL/Tal1 (Figure 2.3A). At this age, the kidney is undergoing formation of nascent endothelial tubes. Note that Nanog is a stem cell marker, and $\mathrm{Hb}$ is widely expressed in the hematopoietic lineage, including long term repopulating HSCs, suggesting that some of these cells 
within the embryonic kidney may not be erythroblasts but earlier hematopoietic precursors.

To identify multipotential and lineage-committed hematopoietic precursors, we performed an in vitro methylcellulose based colony forming cell (CFC) assay using cells from E12.5 kidneys and livers (as controls) of ER-GFP-Cre $/$; $R 26^{Y F P /+}$ mice. During culture, the progenitors differentiated into different types of colonies characterized by their size, morphology and cellular composition. The results showed that cells within these organs formed two types of early stage hematopoietic colonies (CFU-GEMM: containing granulocytes, erythrocytes, monocytes/macrophages and megakaryocytes and CFU-GM: containing granulocytes and macrophages). The YFP reporter was found homogeneously expressed in 25\% of the CFU-GEMM derived from kidney cells (Figure 2.3B), but not in the CFU-GM colonies. In addition, Nanog+, Runx1+ and SCL+ hematopoietic precursors without $\beta$-gal expression were also found either in the metanephric mesenchyme or in forming renal vessels (Figure 2.3A). These results indicated the existence of at least two different early hematopoietic precursors within the embryonic kidney.

To further investigate the contribution of the HSC-SCL precursors to multi-potent hematopoietic precursors in early embryonic kidney, we performed CFC assays using kidney and liver cells from E12.5 HSC-SCL-Cre-ERT+/;R26 $6^{m T m G /+}$ embryos treated with tamoxifen from E9.5 to E11.5. (Figure 2.3C) After 12 days in culture, CFU-GEMM and CFU-GM formed at a rate of 30 colonies per $10^{5}$ kidney cells (Figure 2.3D), suggesting that the developmental stage of hematopoietic precursor cells in 
the early prevascular kidney spans from early multipotent to later myeloid precursors. In this model, GFP reporter expression was observed in approximately $20 \%$ of the colonies from the kidney and liver (Figure 2.3D). Overall this experiment demonstrates that the embryonic kidney possesses multi-potent hematopoietic precursors derived from HSC-SCL+ cells.

By E13.5, the EC-SCL precursors (labeled at E6.5 or from E9.5 to E12.5) gave rise to a subset of blood-like cells in the kidney (Figure 2.4A). Thus, we investigated whether these cells were hematopoietic precursors. Within the kidney, cells derived from the EC-SCL progenitors labeled from E9.5 to E12.5 showed expression of $H b$, SCL and Runx1 (Figure 2.3F). When the progenitors were labeled at E6.5, the E13.5 kidneys showed $\beta$-gal+ cells co-expressing $S C L$ and $A C E$ (Figure 2.4B). These results suggest that the EC-SCL progenitors in the early embryonic kidney generate hematopoietic cells.

Next, to study the potency of the hematopoietic cells derived from renal EC-SCL precursors, we performed CFC assays from kidneys and liver of E12.5 EC-SCL-Cre-

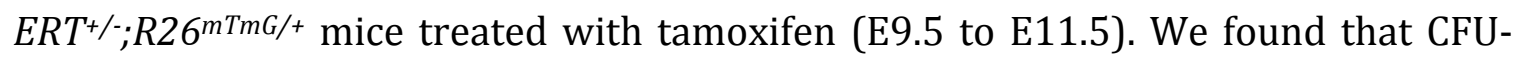
GM and CFU-GEMM formed at a rate of $25.3( \pm 4)$ colonies per $10^{5}$ kidney cells and $15.4 \%$ of the colonies expressed GFP (Figure 2.4E).

\subsection{Hemo-Vasculogenesis In the Embryonic Kidney}

While the aforementioned data indicates the existence of hematopoietic precursors in the early embryonic kidney, to further assess whether those precursors originate and differentiate in situ, we used an in vivo cross-transplantation model, which 
allows the development of the vasculature in the embryonic kidney. Prevascular kidneys from E12.5 EC-SCL-Cre-ERT ${ }^{+/ ;} ; 26^{\text {LacZ/+ }}$ mice and their Cre- siblings were transplanted under the kidney capsule of adult wild type hosts. Then the hosts were treated with tamoxifen for 7 days to induce Cre expression in the EC-SCL precursors present at E12.5 in the transplanted embryonic kidneys. As shown in Figure 2.5, the transplanted kidneys developed vessels with $\beta$-gal+ ECs and vascular SMCs coating (Figure 2.5A). Hematopoietic cells derived from EC-SCL precursors were also identified in the vessels of the transplanted kidney (Figure 2.5A), confirming that those hematopoietic precursors originated in situ. In transplanted kidneys from E12.5 HSC-SCL-Cre-ERT+/;R26 LacZ/+ mice, HSC-SCL precursors gave rise to a small population of renal vascular ECs (Figure 2.5B). The transplanted kidneys from Crecontrol mice of both strains developed mature arterioles and nephrons similar to the Cre+ ones but, as expected, lacked $\beta$-gal+ cells. These results further supported the notion that the embryonic kidney has the capability to establish its own vasculature and blood cells at an early stage.

To investigate whether intrinsic renal EC-SCL precursors are required for the development of the kidney vasculature, we designed experiments to ablate renal ECSCL in the early developing kidney. We crossed EC-SCL-Cre-ERT mice with R26 $6^{\text {DTA/DTA }}$ mice, which express diphtheria toxin subunit A (DTA) in the presence of Cre recombinase resulting in specific cell ablation. To overcome the embryonic lethality resulting from ablation of EC we took advantage of the crosstransplantation technique described above. We transplanted E12.5 kidneys of ECSCL-Cre-ERT+/;R26LacZ/+; R26 $6^{\text {DTA/+ }}$ mice (and DTA negative controls) under the 
kidney capsule of adult $R 26^{\mathrm{mTmG} / m T m G}$ host mice (which expresses ubiquitously cell membrane-localized red fluorescence) and induced DTA expression in EC-SCL precursors after transplantation. In the DTA+ transplanted kidneys, there were significantly reduced numbers of $\beta$-gal+ ECs (Figure 2.5C). Measurement of area fraction (\%) of PECAM-1+ cells in frozen sections of transplanted kidneys showed that the EC area fraction of DTA+ kidneys (1.8\%) was reduced when compared to controls (3.98\%) (Figure 2.5D). Importantly, cells from the host (expressing RFP) do not contribute to the EC endowment in either control or DTA+ transplanted kidneys (Figure 2.5D). These experiments underscore the requirement of intrinsic EC-SCL precursors within the early embryonic kidney for normal organogenesis.

\subsection{Timed Deletion of S1P1 In Renal EC Precursors Results In Abnormal Renal Vascular} Development

To study the role of S1P1 in the development of the kidney vasculature, we induced the deletion of S1P1 in the SCL+ ECs at E10.5 (just before nephrogenesis starts at E11.5) and at E12.5. The iEC-SCL-S1P1KO embryos showed severe dorsal subcutaneous edema, hemorrhages (Figure 2.6A), bradycardia and died around E14.5 to E16.5. To confirm the deletion of S1P1 in endothelial cells we performed RT-PCR for S1P1 in sorted SCL+ ECs (GFP+) from E15.5 kidneys of $i E C-S C L-S 1 P 1 K O$ $m T m G$ and heterozygous Cre+ control mice. (Figure 2.7)

Since at E15.5 the kidney starts to form mature renal arteries and arterioles with SMCs and pericytes coating the EC tubes, we first characterized the kidneys of $i E C$ SCL-S1P1KO mice and their Cre+ control siblings at this age. X-gal staining showed 
striking dilatation of intra-renal arteries and veins in kidneys of $i E C-S C L-S 1 P 1 K O$ mice (Figure 2.6B), accompanied by a marked increase in the number of cells derived from EC-SCL precursors (Figure 2.6C). Co-immunofluorescence staining for PECAM-1 and phosphorylated histone $\mathrm{H} 3$ (pHH3) showed a significant increase of EC proliferation in intra-renal arteries, veins and peritubular capillaries in kidneys of iEC-SCL-S1P1KO mice (Figure 2.6C). No difference was found within the glomerular capillaries (Figure 2.6C).

At E15.5, mature glomeruli appear in the deep renal cortex of both $i E C-S C L-S 1 P 1 K O$ mice and control mice. Capillary shunts (single dilated glomerular capillary lumens) were present within the glomeruli of $i E C-S C L-S 1 P 1 K O$ mice (Figure 2.6D). This phenotype may be due to abnormal development of mesangial cells, which normally support and maintain the structure of the glomerular capillaries as clumps of mesangial cells were present within the abnormal glomeruli (Figure 2.6D).

Unlike previous findings in the aorta of S1P1f/ff; $T i e 2-C r e^{+/-}$mice and S1P1KO mice, immunostaining for $\alpha$-SMA showed that SMCs covered the whole circumference of arteries and arterioles (Figure 2.6D). However their orientation was disturbed with $\alpha$-SMA+ cells aligning irregularly outside of the endothelial tubes (Figure 2.6D).

In addition, lymphatic ECs (LYVE-1+) were absent in the kidneys of $i E C$-SCL-S1P1KO mice (Figure 2.6D). Most of the LYVE-1+ cells were not labeled by X-gal staining in control mice, confirming that the lymphatic endothelium originates from a distinct progenitor (Figure 2.6D). 
3.5 The Kidney Vascular Abnormalities of iEC-SCL-S1P1KO Mice Are Intrinsic And Not Secondary To Extra-Renal Defects

In addition to the kidney, we studied abnormalities in other major organs of the $i E C$ SCL-S1P1KO mice. The most significant abnormalities were found in the heart. The interventricular septum and ventricular myocardium compact layer, especially of the left ventricle, of $i E C-S C L-S 1 P 1 K O$ mice were thinner than those in control mice (Figure 8, A-B). Intra-myocardial vessels of the $i E C$-SCL-S1P1KO mice were dilated (Figure 8C).

To elucidate whether the phenotype observed in the iEC-SCL-S1P1KO mice was intrinsic to the kidney and not secondary to heart failure or other extra-renal anomalies, we transplanted E12.5 kidneys of $i E C-S C L-S 1 P 1 K O$ and control siblings under the kidney capsule of wild type host mice. We induced Cre expression and subsequent S1P1 deletion with simultaneous reporter expression after transplantation. iEC-SCL-S1P1KO transplanted kidneys also showed EC hyperplasia, dilated intrarenal arteries and veins and glomerular capillary shunts (Figure 2.9), suggesting that the abnormal renal vascular development was not secondary to heart failure underscoring the intrinsic role of endothelial S1P1 in kidney vascular development.

\subsection{Kidney Vascular Development Ex Vivo Requires S1P-S1P1 Signaling}

Embryonic kidneys under ex vivo culture conditions are able to develop a normal tubular epithelial compartment (i.e. ureteric bud branches and glomerular epithelial differentiation) but not a normal vasculature. To test whether renal vascular 
development is regulated by the S1P-S1P1 signaling, we performed embryonic kidney cultures exposed to synthetic S1P and S1P1 inhibitor VPC (23019) treatment. Four groups of E12.5 kidneys were cultured and treated with vehicle, S1P, S1P and VPC and VPC alone, respectively, for 72 hours.

Immunostaining for PECAM-1, as expected, showed that untreated (vehicle) embryonic kidneys did not develop an organized vasculature. Similarly, treatment with VPC alone resulted in abnormal disorganized endothelium of intra-renal vessels. However, the kidneys with S1P treatment developed a normal thin layer of ECs, which was attenuated by adding VPC (Figure 2.10A and B). All the cultured kidneys lacked small vessels, including arterioles, peritubular capillaries and glomerular capillaries (Figure 2.10A and B) and mesangial cells (Figure 2.10C).

Moreover, abundant PDGFR $\beta$ + cells, presumably SMC precursors, were identified in close association with ECs in kidneys of the 4 groups (Figure 2.10C). The most $\alpha$ SMA+ cells were found widely distributed in the kidneys with S1P treatment (not shown), compared to the few ones found in the other groups, in which PDGFR $\beta+$ cells do not acquire the $\alpha$-SMA marker, indicating lack of maturation of the SMC coating of the vessels in culture.

\section{Discussion}

In this study we show that the early embryonic kidney possesses $S C L+$ hemovascular precursors that contribute to the intrinsic generation of endothelium concomitant with blood generation, a process that was previously defined as hemovasculogenesis. $18,103,104$ We found that within the kidneys, $S C L+$ precursors give rise 
to hemogenic ECs of renal arteries, veins, arterioles, peritubular capillaries and glomerular capillaries. Furthermore we show that S1P1 expressed in $S C L+$ derived ECs controls the development and assembly of the renal vasculature (Figure 2.11).

Using graft studies and multiple markers, including Flk1, Flt1 and Tie1, other groups have identified early EC precursors for the glomerular microvessels in the rodent kidney. ${ }^{15,105,106}$ Whereas our studies suggest that $S C L+$ precursors originate locally in the kidney, the fact that we found cells derived from the $S C L+$ precursors labeled at either E6.5 or E9.5, before the kidney starts to form, indicates the possibility that precursors from the yolk sac and AGM may have seeded the intermediate mesoderm before the condensation and development of the "avascular" metanephric mesenchyme.

Our fate tracing studies identified $S C L+$ derived hemogenic ECs and hematopoietic precursors in E12.5 and E13.5 kidneys by immunostaining, CFC assay and crosstransplantation studies. During early embryogenesis, hematopoiesis was found to occur in organs other than the yolk sac or the AGM region. A recent study showed that the mouse embryonic head also functions as a hemogenic organ to a similar extent as the AGM region, which suggests broader potential hemogenic areas in the mouse embryo; in agreement with previous studies showing that hemovasculogenesis also occurs in the head mesenchyme of early embryos. ${ }^{18,40}$ The kidney is a major hemogenic organ in teleosts, such as zebra fish. Our results suggest that the mouse kidney also functions as a temporary hemogenic organ via hemo-vasculogenesis during early embryogenesis. Although we did not identify $S C L+$ lineage derived blood cells in the embryonic kidneys at later stages (E15.5 and 
E17.5, data not shown), it is possible that they directly joined the blood circulation at earlier stages and homed to the classic hematopoietic sites such as fetal liver, spleen and bone marrow. In fact the connection between the embryonic kidney and peripheral circulation is already established at E15.5. In our colony forming assays, not all colonies were labeled by reporter expression. The unlabeled colonies may originate from earlier hematopoietic precursors or may have escaped the induction by tamoxifen. These results are in agreement with our fate tracing studies of EC-SCL and HSC-SCL precursors using X-gal staining and immunostaining. Interestingly, we found that the HSC-SCL precursors give rise not only to blood cells but also to a small population of ECs within the embryonic kidney suggesting the early embryonic presence of endothelial heterogeneity within the same organ.

The temporary hemogenic function of the embryonic kidney may serve as a smooth transition before the establishment of the connection to the general circulation, as the formation of a local vascular system also requires blood to generate regulatory factors, such as S1P. A recent study has shown that deletion of red blood cell specific sphingosine kinases 1 and 2 (Sphk1 and Sphk2) in mice results in abnormal vascular development, which can be rescued by treatment with an S1P1 receptor agonist. ${ }^{58}$ Thus the $S C L+$ hemogenic precursors in the embryonic kidney give rise to a small population of blood cells, which in turn may function as crucial regulators for the concomitant development of the kidney vasculature. Interestingly, the development of the renal vascular tree is hindered in the culture system. The smaller vessels and glomerular capillaries in the nephrogenic zone are missing in the cultured kidneys even with S1P treatment, suggesting that the development of these vessels requires 
other factors, such as blood flow, angiogenic factor, such as VEGF-A and a hypoxic micro-environment.

Recent studies show that S1P1 inhibits sprouting angiogenesis of retinal vessels in mice by regulating interactions between VE-Cadherin and VEGFR2 during vascular development. ${ }^{55,56}$ Our study indicates that S1P1 functions as an inhibitor of angiogenesis in a cell autonomous manner within the kidney. E14.5-E15.5 iEC-SCLS1P1KO embryonic kidneys and cross-transplanted kidneys displayed increased EC proliferation and EC hyperplasia. In culture, the embryonic kidneys treated with VPC and S1P+VPC developed hyperplastic ECs of intra-renal vessels, whereas in those treated with S1P the EC layer was thin. Since S1P is mainly generated by erythrocytes and ECs, and the plasma level of S1P is higher than that in the tissue, lack of S1P normally supplied by the circulating blood may lead to the EC hyperplasia in cultured kidneys.

Whereas there was not a significant increase of proliferation in the developing glomerular capillaries of $i E C-S C L-S 1 P 1 K O$ kidneys, they developed capillary shunts with abnormal mesangial cells, suggesting a distinct role of S1P1 in the development of glomerular capillaries in comparison with other renal blood vessels. Mesangial cells play an important role in development and stabilization of the glomerular capillary loops. ${ }^{107-109}$ It has been hypothesized that the mesangial cell splits the single vessel into multiple parallel branches, which forms the capillary tufts. ${ }^{109}$ However, exactly how the capillary loops form and the molecular cues guiding this process are unknown. Previous studies showed that knockout of several transcription factors expressed in podocytes (Pod1, Foxc2, Limx1b and Krm1/MafB) 
resulted in decreased number of capillary loops and abnormal mesangium similar to what we observed in our study. ${ }^{110-113}$ Therefore S1P1 may be involved in the interactions among podoctyes, ECs and mesangium to regulate the development of the glomerular capillary loops. Whether S1P1 operates through the transcription factors mentioned above requires further investigation.

Deletion of S1P1 in EC precursors did not result in absence of mural cells of the renal vessels, however, the SMC layer was disorganized, and the vessels of cultured kidneys lacked SMC coating. Although S1P treatment to the kidneys in culture increased the number of $\alpha$-SMA+ cells, the kidneys still showed vascular SMC recruitment defects. Although this result could be due to the culture system, the overall agreement between the in vivo and in vitro studies suggests that in fact S1PS1P1 signaling controls the recruitment of mural cells during vascular development. Interestingly, the $i E C$-SCL-S1P1KO mice showed abnormal heart development. S1P1 is ubiquitously expressed in multiple cardiac cell types, including cardiomyocytes, fibroblasts, SMCs and ECs. ${ }^{114}$ Furthermore, S1P1, S1P2 and S1P3 are expressed in cardiomyocytes and mediate cardioprotection from hypoxia. ${ }^{114-116}$ Until now, the role of endothelial S1P1 in the heart was unclear. Our study suggests that S1P1 expressed in cardiac ECs may not only regulate vascular development but also play an important overall role in heart development.

An additional finding from our study is the lack of lymphatic vessels in the kidneys of $i E C$-SCL-S1P1KO mice. Previous studies have shown that lymphatic ECs start to appear in the mouse kidney at E13.5 ${ }^{13}$ and that multiple cell types contribute to the 
their development, including venous derived ECs, mesenchymal cells and hematopoietic cells. ${ }^{117-119}$ Lack of reporter expression in LYVE-1+ lymphatic ECs of

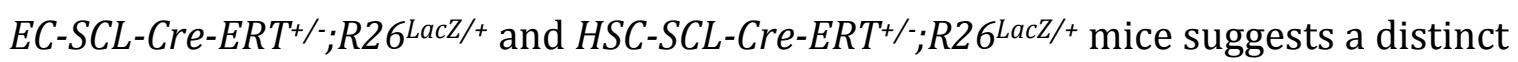
origin of lymphatic ECs from non SCL+ precursors. However, S1P1 expressed in vascular ECs still seems to play a key role in development of the renal lymphatic vessels. The mechanism involved remains to be investigated.

We showed that $i E C$-SCL-S1P1KO mice developed severe edema. The accumulated interstitial fluid caused by absence of lymphatic vessels, the abnormal SMC coating of blood vessels and abnormal heart function may have increased hydrostatic pressure and therefore contributed to prominent vessel dilation, hemorrhages and dorsal edema.

In summary, as depicted in Figure 2.11, our data showed that vascular endothelium and blood cells originate in situ from SCL+ precursors in the mouse prevascular embryonic kidney. The S1P-S1P1 signaling pathway controls the development and assembly of the kidney vasculature during mouse early embryogenesis.

\section{Methods}

\subsection{Mice}

ER-GFP-Cre, ${ }^{120}$ EC-SCL-Cre-ERT, ${ }^{99}$ HSC-SCL-Cre-ERT, ${ }^{100}$ and Hoxb7-Cre ${ }^{121}$ mice have been previously described. B6.129S4-Gt(ROSA)26Sortm1Sor/J (R26LacZ/LacZ) 122, B6.129(Cg)-Gt(ROSA)26Sortm4(ACTB-tdTomato,-EGFP)Luo $/ \mathrm{J} \quad\left(R 26^{m T m G / m T m G}\right){ }^{123}$ and B6.CgGt(ROSA)26Sortm3(CAG-EYFP)Hze $/ \mathrm{J}\left(R 26^{\text {YFP/YFP })}{ }^{124}\right.$ reporter mice were used to trace the fate of erythroblasts, EC-SCL and HSC-SCL precursors. 


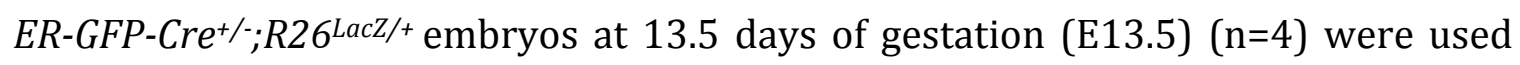
for tracing the fate of erythroblasts.

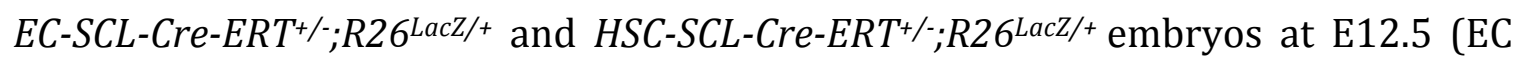
n=22, HSC n=9), E13.5 (EC n=11, HSC n=11), E15.5 (EC n=3, HSC n=2) and E17.5 (EC $\mathrm{n}=5, \mathrm{HSC} \mathrm{n}=2)$ were used for fate tracing studies. $E C-S C L-C r e-E R T^{+/} ; R 26^{m T m G /+}(\mathrm{n}=6)$

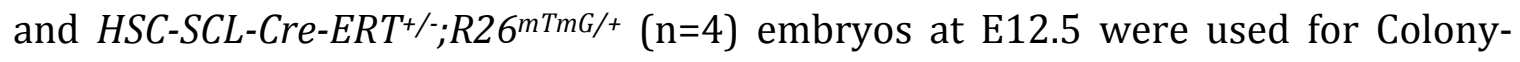
forming cell assays. Pregnant mice were injected with tamoxifen $(2 \mathrm{mg} / 30 \mathrm{~g}$ body weight) intraperitoneally (i.p.).

EC-SCL-Cre-ERT mice were crossed to B6.129-GT(ROSA)26SSortm1(DTA)Lky/J mice $\left(R 26^{\text {DTA/DTA }}\right)^{125}$ and $R 26^{\text {LacZ/LacZ }}$ mice to investigate whether ablation of EC precursors affects kidney development. EC-SCL-Cre-ERT+/;R26 LacZ/+ (n=9), EC-SCLCre-ERT $\%$;R26LacZ/+ $(\mathrm{n}=4), H S C-S C L-C r e-E R T^{+/ ;} / R 26^{L a c Z /+}(\mathrm{n}=12), H S C-S C L-C r e-E R T \%$ ;R26 $6^{\text {LacZ/+ }} \quad(\mathrm{n}=4), \quad$ EC-SCL-Cre-ERT $T^{+/} ; R 26^{\text {LacZ/+;R26 }} 6^{\text {DTA/+ }} \quad(n=4), \quad$ EC-SCL-Cre-ERT\%

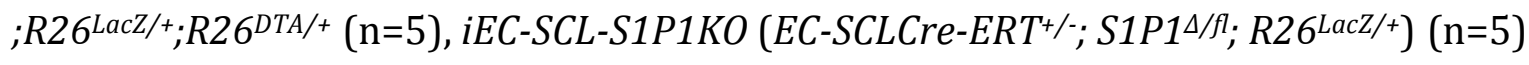
and Cre+ control siblings (EC-SCL-Cre-ERT $\left.T^{+/ ;} S 1 P 1^{f /+} ; R 26^{\text {LacZ/++}}\right)(\mathrm{n}=4)$ embryos at E12.5 were used for cross-transplantation experiments.

E12.5 embryos ( $\mathrm{n}=14$ ) from the cross between Hoxb7-Cre mice ${ }^{121}$ and R26LacZ/LacZ mice were used for embryonic kidney culture studies.

To generate $i E C$-SCL-S1P1KO mice, first we crossed S1P1f/ff53 mice to EIIA-Cre ${ }^{+/-}$ mice ${ }^{126}$ to obtain EIIACre ${ }^{+/ ;}$S $1 P 1^{\Delta /+}$ mice. Then this mouse line was crossed to wt Bl6 mice to remove the EIIA-Cre. The S1P1//+ mice were born at the expected Mendelian ratio and were fertile. These mice were crossed with EC-SCL-Cre-ERT mice to 
generate $E C$-SCLCre-ERT $T^{+/ ;} S 1 P 1^{\Delta /+}$ mice which next were crossed to the S1P1f/ff; R26 $6^{\text {LacZ/LacZ }}$ mice to generate the EC-SCLCre-ERT ${ }^{+/ ;} S 1 P 1^{\Delta / f l}$; R26 ${ }^{\text {LacZ/+ }}$ (iEC-SCLS1P1KO) mice and their control siblings (EC-SCLCre-ERT $T^{+/ ;}$S1P1fl/+; R26 $6^{L a c Z /+}$ ).

To assess the deletion of S1P1 we crossed EC-SCL-Cre-ERT $T^{+/ ;} S 1 P 1^{\Delta /+}$ to S1P1fl/f; $R 26^{m T m G / m T m G}$ to generate $i E C-S 1 P 1 K O-m T m G\left(E C-S C L C r e-E R T^{+/-;} S 1 P 1^{\Delta / f l} ; R 26^{m T m G /+}\right)$ mice, with GFP reporter expression in ECs with S1P1 deletion and heterozygous controls with GFP reporter expression in ECs with one intact S1P1 allele (EC-SCLCre-ERT $T^{+/ ;} ; S 1 P 1^{\Delta /+} ; R 26^{m T m G /+}$ ). Genomic DNA from embryonic tissue was genotyped for Cre, wild type S1P1 (S1P1 wt) and S1P1 deletion (S1P1A) as previousely described. ${ }^{54,}{ }^{99}$ Four genotypes, including conditional S1P1 knockout, Cre+ control, S1P1 with one functional allele and wild type (wt) mice were obtained. Each genotype had equal opportunity based on Mendelian Ratio (25\%). Conditional S1P1 knockout (cKO) mice were Cre positive, S1P1A positive and S1P1wt negative; Cre+ control mice were Cre positive, S1P1 $\Delta$ negative and S1P1wt positive; the Cre-control mice were Cre negative, S1P1 positive and S1P1wt positive; the wt mice were Cre negative, S1P1 $\triangle$ negative and S1P1wt positive. The genotyping results are shown in Table 2.1.

To minimize the toxicity and maximize the efficiency of Cre induction, 137 embryos from 21 litters were studied with different dose and number of injections. We found that mice with two injections (one at E10.5 and one at E12.5) $(1 \mathrm{mg} / 30 \mathrm{~g}$ per injection) showed less toxicity and sufficient Cre recombination. 110 embryos of 15 litters at ages ranging from E14.5 to E16.5 (E14.5 n=28, E15.5 n=65, E16.5 n=17) 
were treated following this protocol. Based on genotyping results, $27.3 \%$ of the embryos (30 of 110) were conditional S1P1 knockout, which matched the Mendelian ratio (25\%). 53.3\% of the conditional S1P1 knockout mice were dead at the time of harvesting (Table 2.1).

Table 1. Genotyping results of S1P1 conditional knockout embryos

\begin{tabular}{lccccc}
\hline & $c K O$ & $\begin{array}{c}\text { Cre+ } \\
\text { control }\end{array}$ & $\begin{array}{c}\text { Cre- } \\
\text { control }\end{array}$ & WT & Total \\
\hline $\begin{array}{l}\text { Number of } \\
\text { embryos }\end{array}$ & 30 & 27 & 23 & 30 & 110 \\
$\begin{array}{l}\% \text { of embryos } \\
\begin{array}{l}\text { Number of dead } \\
\text { embryos }\end{array}\end{array}$ & $27.3 \%$ & $25.6 \%$ & $20.9 \%$ & $27.3 \%$ & $100 \%$ \\
$\begin{array}{l}\% \text { of dead } \\
\text { embryos }\end{array}$ & 16 & 1 & 0 & 1 & 18 \\
\hline
\end{tabular}

All procedures were performed in accordance with the Guiding Principles for Research Involving Animals and Human Beings by the American Physiological Society and were approved by the University of Virginia Animal Care Committee.

\subsection{Cell sorting and RT-PCR}

Whole kidneys from E15.5 iEC-S1P1KO-mTmG (EC-SCLCre-ERT+/; S1P14/fl; R26 $\left.{ }^{m T m G /+}\right)$ mice and their Cre+ control siblings (EC-SCLCre-ERT $T^{+/ ;}$; $\left.1 P 1^{\Delta /+} ; R 26^{m T m G /+}\right)$ (treated with tamoxifen at E10.5 and E12.5 by maternal injections) were micro-dissected and enzymatically dissociated to isolate single cells. GFP+ cells from $i E C-S 1 P 1 K O-$ mTmG and control kidneys were sorted and collected using a Becton Dickinson Influx cell sorter. 
Total RNA extraction and cDNA preparation from sorted cells were performed using a FastLane Cell cDNA kit from QIAGEN, according to the manufacturer's instructions. Quantitative PCR was performed in a CFXConnect system (Biorad, Hercules, CA) using a QuantiTect SYBR Green PCR kit from QIAGEN, following the manufacturer's instructions. The primers used for RT-PCRs were:

S1P1 forward 5'-AACTTTGCGAGTGAGCTGGT-3', S1P1 reverse 5'CTAGAGGGCGAGGTTGAGTG-3' (product size: 227bp), GAPDH forward 5'TTGATGGCAACAATCTCCAC-3' and GAPDH reverse 5'-CGTCCCGTAGACAAAATGGT-3' (product size: $125 \mathrm{bp}$ ). $\mathrm{PCR}$ conditions were: $95^{\circ} \mathrm{C}, 58^{\circ} \mathrm{C}, 72^{\circ} \mathrm{C}, 45$ cycles.

\subsection{Immunohistochemistry and X-gal Reaction}

$7 \mu \mathrm{m}$ sections of $4 \%$ PFA fixed and cryo-embedded tissues were subjected to X-gal staining and/or immunostained following standard protocols. ${ }^{127}$ The antibodies used were anti- $\beta$-gal (1:1000 dilution, Abcam, ab134435), anti- $\alpha$-SMA (1:10000 dilution, Sigma, a2547), anti-Hb (1:500 dilution, DAKO, A0118), anti-Nanog (1:100 dilution, Abcam, ab84447), anti-SCL (1:100 dilution, Abcam, ab75739), anti-Runx1 (1:500 dilution, Abcam, ab23980), anti-ACE (1:200 dilution, Abcam, ab2092), antiLYVE-1 (1:1000 dilution, Abcam, ab14917), anti-ppH3 (1:100 dilution, Cell Signaling \#9701S), goat anti-PECAM-1 (1:200 dilution, Santa Cruz, sc1505, for Figure 4 and supplemental Figure 3) and rabbit anti-PECAM-1 (1:500 dilution, Santa Cruz, sc1506-R). Immunofluorescence was performed using Alexa Fluor 568 donkey antirabbit IgG $(\mathrm{H}+\mathrm{L})(\mathrm{SCL}, \mathrm{pHH})$, Alexa Fluor 488 goat anti-chicken IgG $(\mathrm{H}+\mathrm{L})(\beta$-gal), Alexa Fluor 488 donkey anti-mouse IgG (H+L) (ACE), Alexa Fluor 488 donkey anti- 
rabbit IgG (H+L) (PECAM-1) and Alexa Fluor 488 donkey anti-goat IgG $(\mathrm{H}+\mathrm{L})($ PECAM-1) secondary antibodies (1:500 dilution; Life Technologies, Carlsbad, CA). Images were obtained using a Leica DFC310 FX digital camera connected to a Leica DFC 480 microscope.

\subsection{Colony forming cell assays}

Kidney and liver cells were harvested from E12.5 mouse embryos and disaggregated with collagenase (M7902, StemCell Technlogies) at $37^{\circ} \mathrm{C}$. The single cells were diluted in Iscove's MDM with 5\% FBS and grown in Methocult GF (M3434, StemCell Technlogies) for assessment of mouse hematopoietic progenitors. Cells in Methocult were plated in duplicate samples at $1 \times 10^{5}$ cell per $30 \mathrm{~mm}$ dish. After incubation for 12 days at $37^{\circ} \mathrm{C}$ in $5 \%$ humidified $\mathrm{CO}_{2}$, the dishes were scored for colony-forming units (CFUs) according to standard criteria. CFU-GEMM colonies are large colonies containing $>500$ cells, with a highly dense core (red or brown color) and indistinct border between the core and peripheral cells. CFU-GM colonies contain $>30$ cells with dense cores (without red or brown). Individual cells can be distinguished at the edge of the colonies. (Described in the Manual: Mouse ColonyForming Unit (CFU) Assays Using MethoCult ${ }^{\mathrm{TM}}$, StemCell Technologies).

\subsection{Embryonic Kidney Cross-transplantation}

The cross-transplantations of embryonic kidneys under the kidney capsule of adult hosts were performed as described previously. ${ }^{101}$ The host mice were anesthetized with tribromoethanol $(0.25 \mathrm{mg} / \mathrm{g}$ body weight). The host kidney capsule was incised with Vannas scissors, and the incision was extended with a 27-gauge needle. 
Embryonic kidneys were freshly micro-dissected and placed under the renal capsule of adult wild type or fluorescent reporter hosts via the capsular incision. After surgery, host mice were injected with analgesic drug buprenorphine $(0.2 \mathrm{mg} / \mathrm{kg}$ body weight) i.p. daily for 2 days and tamoxifen ( $1 \mathrm{mg} / 30 \mathrm{~g}$ body weight) i.p. daily for 7 days. The transplanted embryonic kidneys within the host kidneys were harvested and fixed in 4\% PFA followed by X-gal staining and/or immunohistochemistry.

\subsection{Measurement of endothelial area fraction in the transplanted kidneys}

To measure the endothelial cell (EC) area fraction, we performed immunofluorescence for PECAM-1 (green) on frozen sections (6 sections/ animal) of transplanted kidneys from control (EC-SCL-Cre-ERT $\left.{ }^{+/ ;} ; R_{2} 6^{\mathrm{LacZ} /+}\right)(\mathrm{n}=3)$ and DTA+ $\left(E C-S C L-C r e-E R T^{+/ ;} ; R 26^{L a c Z /+} ; R 26^{D T A /+}\right)(\mathrm{n}=3)$ mice. Nuclei were co-stained with DAPI (blue). Images were processed with Image J to select stained endothelial areas occupied by stained pixels and whole transplanted kidney area. Background staining was manually excluded. The EC area fraction was expressed as percentage:

$$
\text { EC area fraction }(\%)=\frac{P E C A M-1 \text { Stained area }}{\text { Total transplated kidney area }}
$$

\subsection{Measurement of endothelial proliferation}

Quantification for endothelial proliferation was performed on kidney sections from E14.5 iEC-SCL-S1P1KO mice and control siblings, by scoring number of cells with ppH3 (RFP+) expression in the nucleus and PECAM1 (GFP+) expression on the cell membrane. The total area of kidney sections was measured using Image J software. 
5.8 Measurement of Thickness of Septum and Left Ventricle Wall of E15.5 iEC-SCLS1P1KO and control Mice

Longitudinal $5 \mu \mathrm{m}$ heart sections near the central conduction system from control (Ctrl) $\left(E C-S C L-C r e-E R T^{+/ ;}\right.$S1P1fl/+; R26 $\left.6^{L a c Z /+}\right)(\mathrm{n}=4)$ and iEC-SCL-S1P1KO (EC-SCL-Cre$\left.E R T^{+/ / ;} S 1 P 1^{1 / f f} ; R 26^{\text {LacZ/+ }}\right)(\mathrm{n}=4)$ mice were used to measure the thickness of septum and left ventricle wall using Image J software. The thickest parts in the septum and left ventricle compact myocardium were selected for measuring wall thickness.

\subsection{Embryonic Kidney Culture}

E12.5 kidneys from Hoxb7-Cre+/;R26 LacZ/+ mice were dissected in DMEM-Ham' F-12 medium with $1 \%$ FBS and cultured on filters in 24 well plates with $800 \mu$ of culture medium (DMEM/Ham' F-12 medium with 1\%FBS, 1\% Hepes, 1\% antibiotic antimycotic and $0.5 \%$ ITS) plus: (1) vehicle (3\% fatty acid free BSA $+5 \mathrm{mmol} / \mathrm{L}$

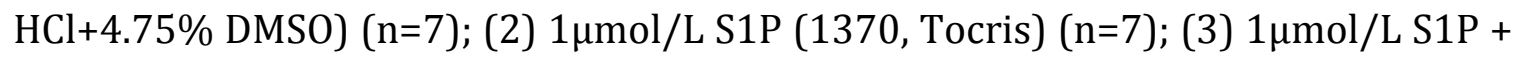

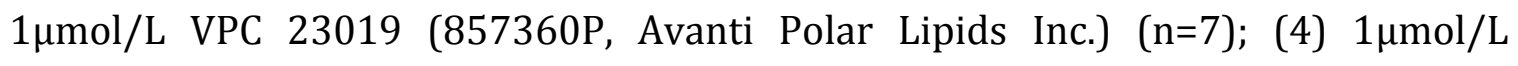
VPC23019 (n=6). Medium was changed daily. Kidneys were harvested after 72 hours and processed for whole-mount X-gal staining followed by post fixation, embedding, sectioning and immunostaining as previously described.127

\subsection{Statistical Analysis}

Data are shown as mean \pm SEM. Statistical analysis was carried out by Student's $t$ test. A $\mathrm{p}<0.05$ was considered significant. 


\section{Figures and figure legends}

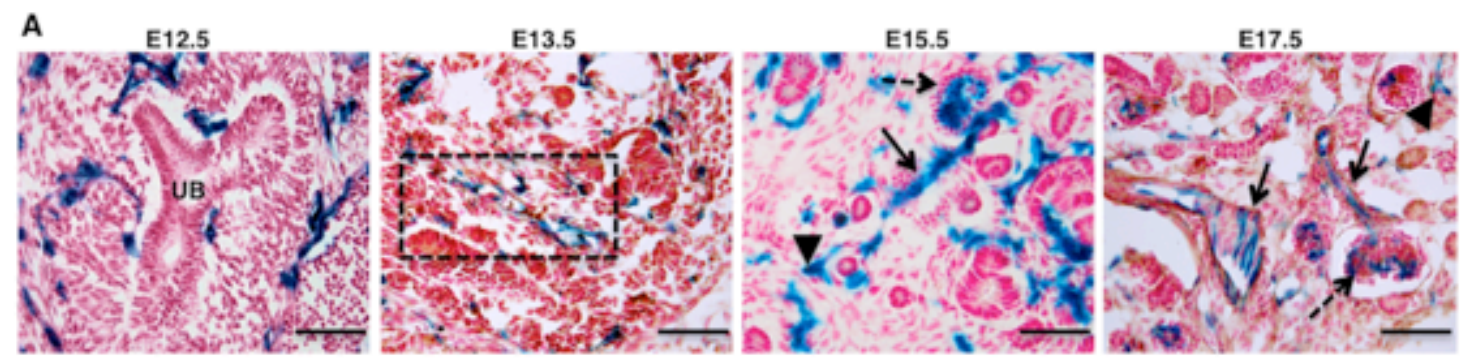

B

E17.5 EC-SCL-Cre-ERT ${ }^{*} ;$ R2 $^{\mathrm{L} n e Z *}+$ kidney
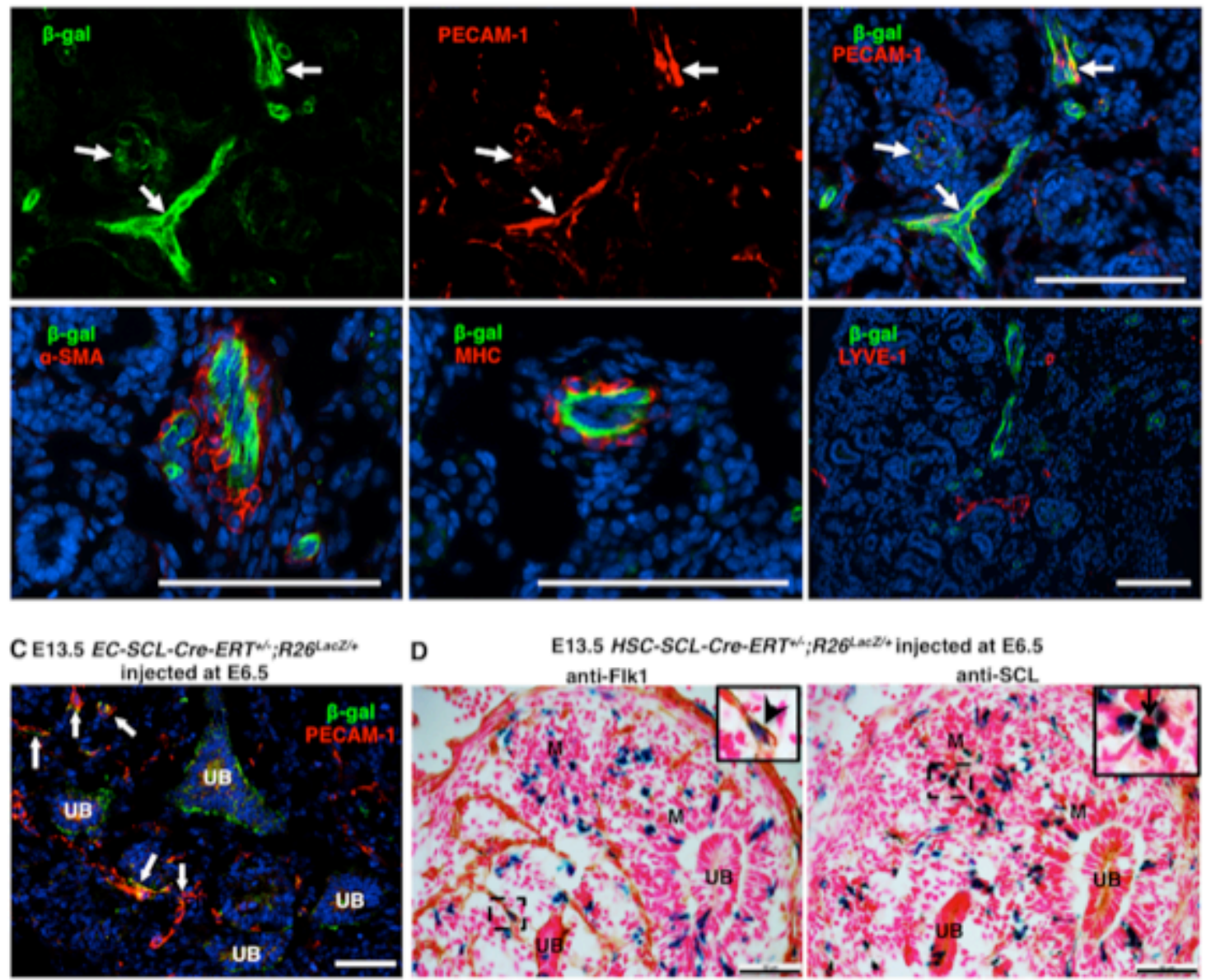

Figure 2.1. $S C L+$ endothelial precursors give rise to renal endothelium but not mural cells in the embryonic kidney. (A) Kidneys of EC-SCl-Cre-ERT+/;R26 ${ }^{\text {LacZ/+ }}$ mice treated with tamoxifen at E9.5 show EC-SCL progenitor derived cells labeled by X-gal staining in blue ( $\beta$-gal+) distributed within the metanephric mesenchyme surrounding the branching ureteric bud (UB) at E12.5, in a nascent 
vessel (dashed rectangle) at E13.5 and in ECs of renal arteries and arterioles (arrows), peritubular capillaries (arrowheads) and glomerular capillaries (dashed arrows) at E15.5 and E17.5. (B) $\beta$-gal+ (green) cells co-express the EC marker PECAM-1 (red)(arrows, upper panels), but not the mural markers $\alpha$-SMA (red) and MHC (red), or the lymphatic EC marker LYVE-1 (red) (lower panels). Nuclei were stained with hoechst in blue. (C) E13.5 EC-SCl-Cre-ERT+/;R26 LacZ/+ mice treated with tamoxifen at E6.5 show that several $\beta$-gal+ cells (green) co-stained with PECAM-1 (red) in the developing vessels (arrows) of the kidney. Nuclei were stained with hoechst in blue. (D) Immunostaining of E13.5 kidney consecutive sections from HSC-

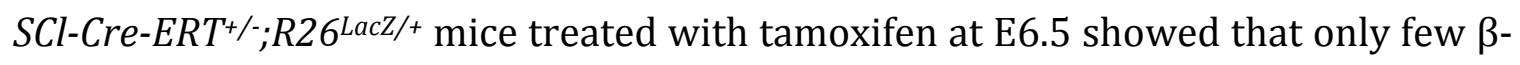
gal+ cells co-expressed the early EC marker Flk1(inset, arrow head). Instead, most of them were distributed in the undeveloped mesenchyme (M) and co-expressed SCL/Tal1 (inset, arrow). UB, ureteric bud. Scale bars: $50 \mu \mathrm{m}$ (A, C and D), $100 \mu \mathrm{m}(\mathrm{B})$ 

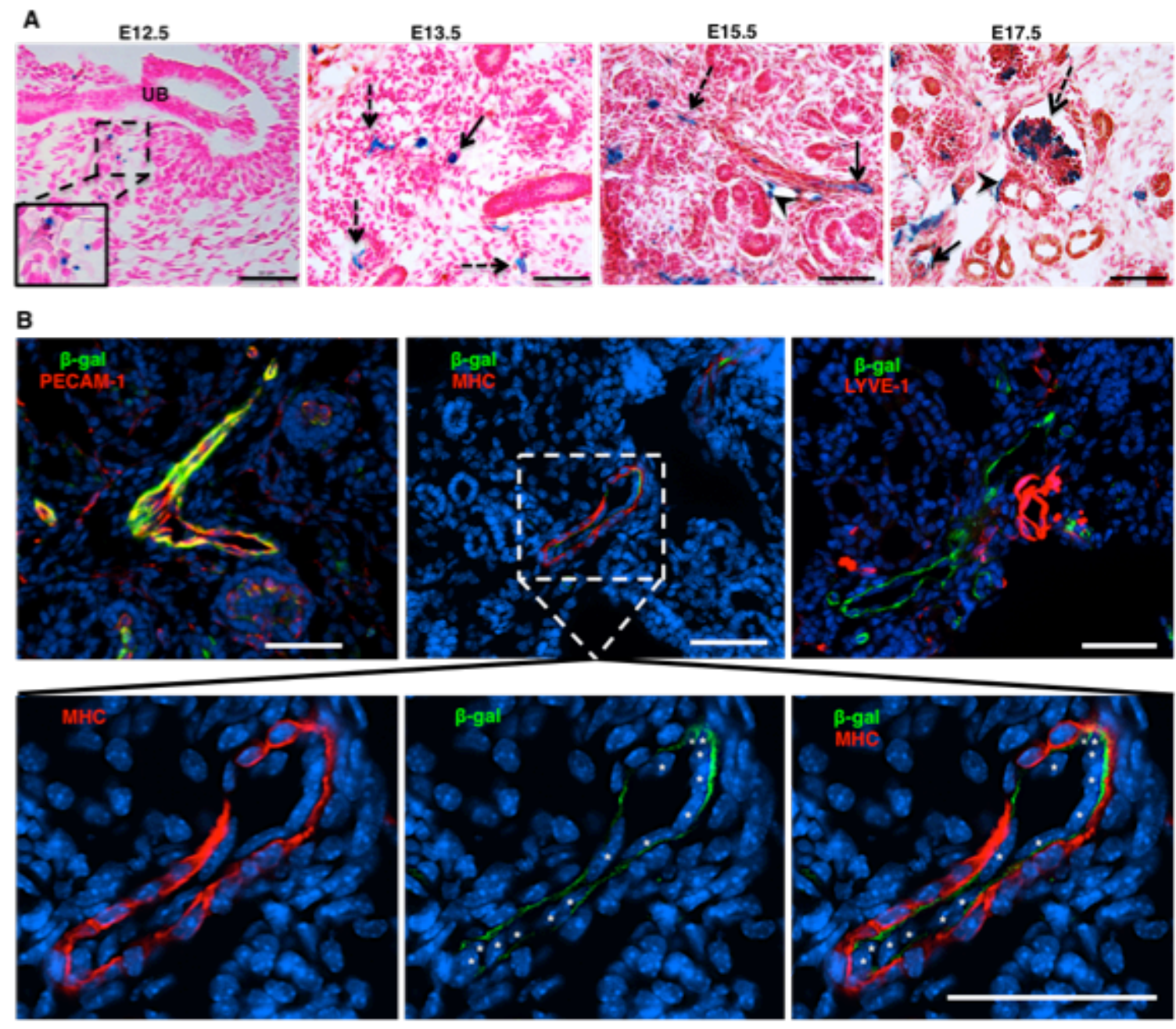

Figure 2.2. $S C L+$ hematopoietic precursors give rise to renal endothelium

but not mural cells in the embryonic kidney. (A) Kidneys of HSC-SCl-Cre-ERT+/-

;R26 $6^{\text {LacZ/+ }}$ mice showing scattered HSC-SCL progenitor derived cells labeled by X-gal in blue ( $\beta$-gal+) close to the ureteric bud (UB) at E12.5 are shown in the inset. $\beta$-gal+ cells adopted round (arrow) and spindle-shape (dashed arrows) configuration in E13.5 kidneys. A subset of $\beta$-gal+ ECs of renal arteries (arrows), veins (arrow heads) and peritubular capillaries (E15.5, dashed arrows) and glomerular capillaries (E17.5, dashed arrow) were found at E15.5 and E17.5. (B) $\beta$-gal+ (green) HSC-SCL lineage cells co-expressed the EC marker PECAM-1 (red), but not the mural marker 
myosin heavy chain (MHC, red) or the lymphatic EC marker LYVE-1 (red). The lower panels show $\beta$-gal and MHC staining at high magnification. Asterisks indicate nuclei of $\beta$-gal+ ECs. Nuclei were stained with hoechst in blue. Scale bars: $50 \mu \mathrm{m}$

A Kidney of E13.5 ER-GFP-Cre;R26R-LacZ mice
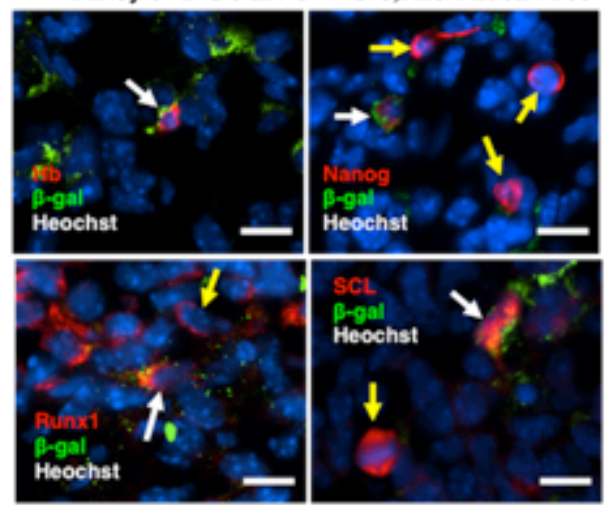

B

CFC of E12.5 ER-GFP.Cre;R26R-YFP

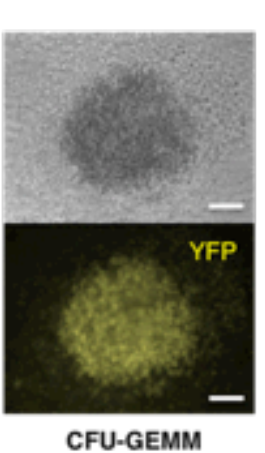

\section{DYFP. CFU-GEMM \\ OYFP+ CFU-GEMM}

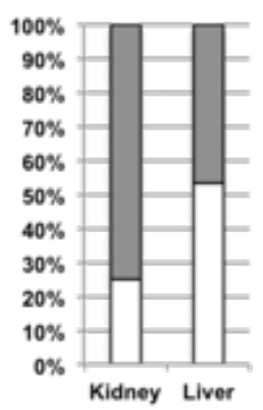

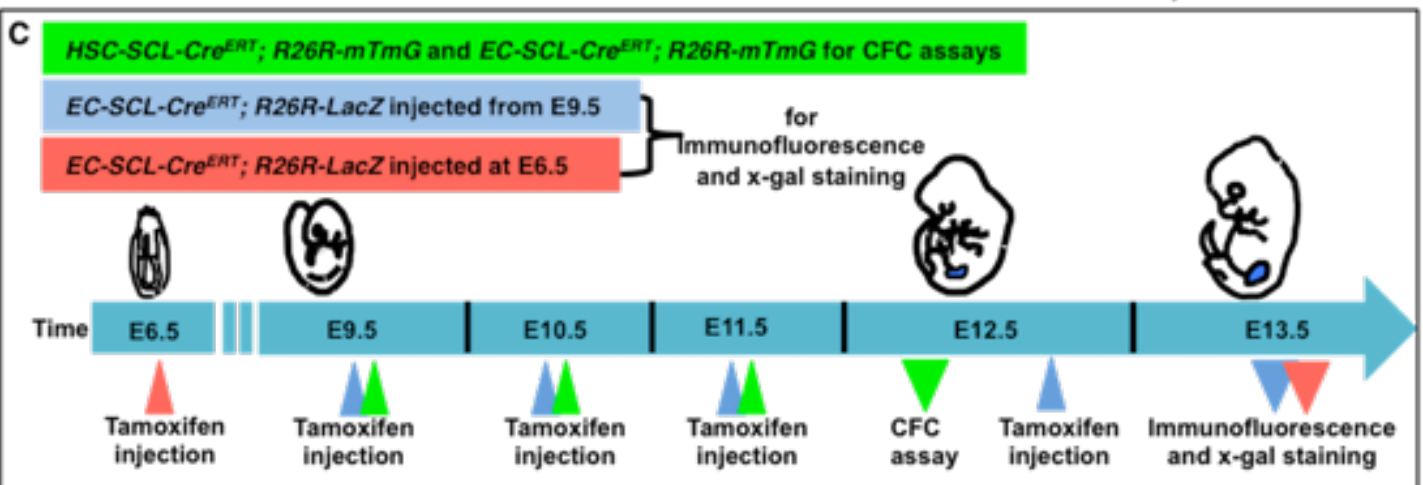

D CFC of E12.5 HSC-SCL-Cre ${ }^{E A T} ;$ R26R-mTmG

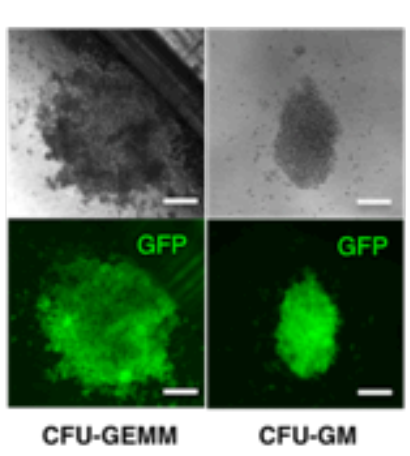

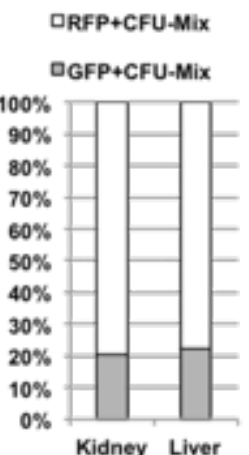

E

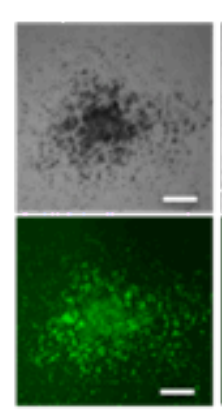

CFU-GEMM

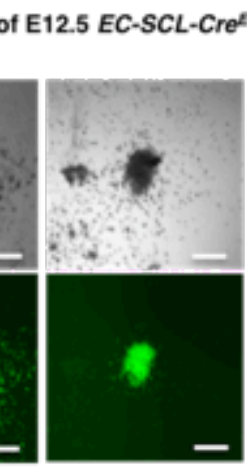

CFU-GM and $x$-gal staining

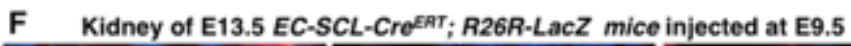
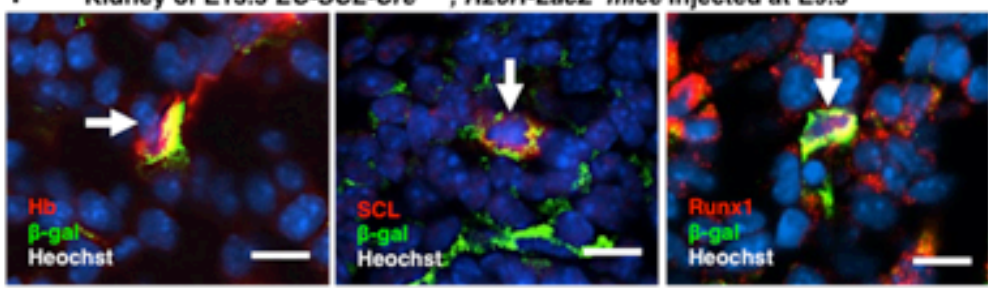
Figure 2.3. The early embryonic kidney possesses hematopoietic precursors.

(A) Double immunofluorescence staining of E13.5 kidney sections from ER-GFP-

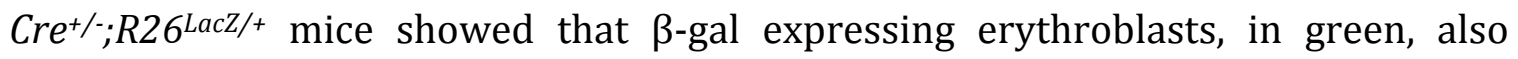
expressed Hb, Nanog, Runx1 and SCL/Tal1 (white arrows). However, some Nanog+, Runx1+ and SCL/Tal1+ cells did not coincidence with labeled erythroblasts (yellow arrows). Nuclei were stained with hoechst in blue. (B) Colony Forming Cell (CFC)

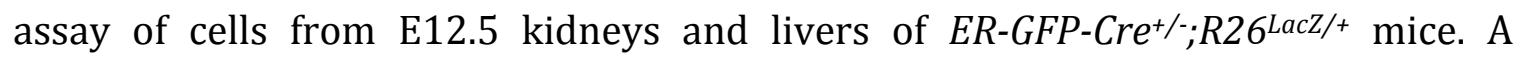
representative photograph of a YFP+ CFU-GEMM grown from kidney cells is shown (Left). The chart shows the percentage of YFP+CFU-GEMM from kidneys $(25 \%, n=3)$ and livers (53.4\%, $\mathrm{n}=3$ ). (C) Timeline of tamoxifen administration in HSC-SCL-Cre-

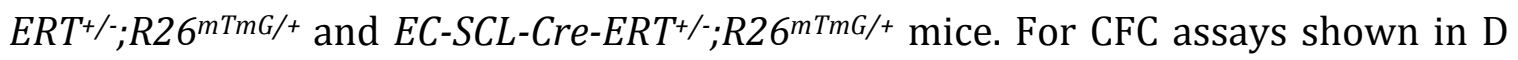

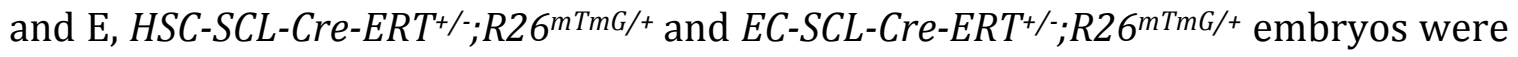
treated with tamoxifen from E9.5 to E11,5 by intraperitoneal maternal injection (green triangles). To label EC-SCL precursors generated after E9.5 (shown in F), ECSCL-Cre-ERT $T^{+/ ;} R 26^{L a c Z /+}$ embryos were treated with tamoxifen daily from E9.5 to E12.5 and harvested at E13.5 (blue triangles). To label EC-SCL precursors present before E9.5 (Supplemental Figure 2B), EC-SCL-Cre-ERT+/;R26 LacZ/+ embryos were treated with a single injection of tamoxifen at E6.5. The embryos were harvested at E13.5 (red triangles). (D) CFC assay of cells from E12.5 kidneys ( $n=4)$ and livers $(\mathrm{n}=4)$ of $H S C$-SCL-Cre-ERT $/ / ; R 26^{m T m G /+}$ mice. CFU-GEMM (left) and CFU-GM (right) colonies grew from all the samples. Bar graph shows the percentage of GFP+ colonies out of total CFU-Mixs grown from kidneys (21\%) and livers (22.2\%). (E) 
CFC assay of cells from E12.5 kidneys $(\mathrm{n}=6)$ and livers $(\mathrm{n}=6)$ of $E C$-SCL-Cre-ERT+/;R26 $6^{m T m G /+}$ mice. CFU-GEMM (left) and CFU-GM (right) colonies grew from all the samples. Bar graph shows that $15.4 \%$ of the colonies grown from kidney cells and $30 \%$ of those from liver cells express GFP. Data was collected from two independent

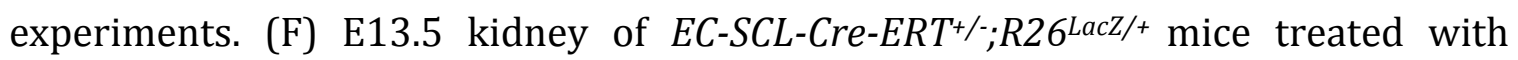
tamoxifen from E9.5 to E12.5. Blood cells derived from $S C L+$ endothelial precursors, in green, coincided by immunofluorescence with $\mathrm{Hb}$, SCL/Tal1 and Runx1 (red, white arrows). Nuclei were stained with hoechst in blue. Scale bars: $10 \mu \mathrm{m}$ (A and F); $50 \mu \mathrm{m}(\mathrm{B}, \mathrm{D}$ and $\mathrm{E})$.
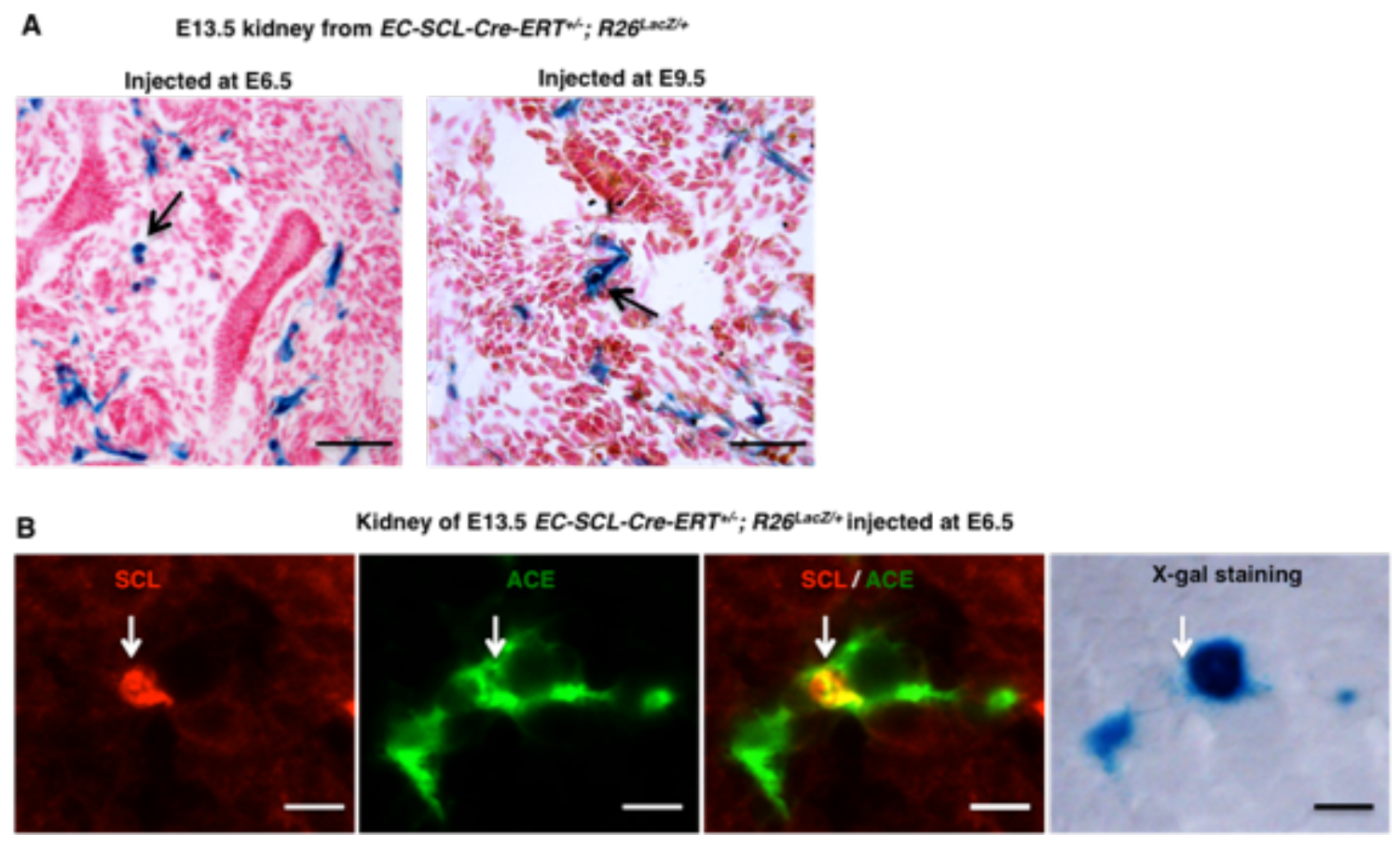

\section{Figure 2.4. The early embryonic kidney possesses hematopoietic}

precursors. (A) E13.5 kidneys showing blood like labeled cells, in blue, derived

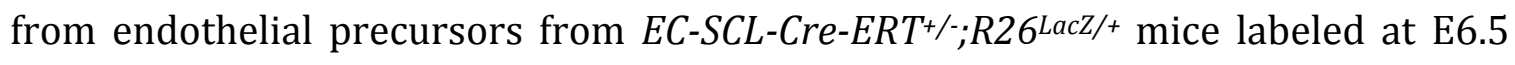
and from E9.5 to E13.5 (black arrows). (B) E13.5 embryonic kidneys from EC-SCL- 


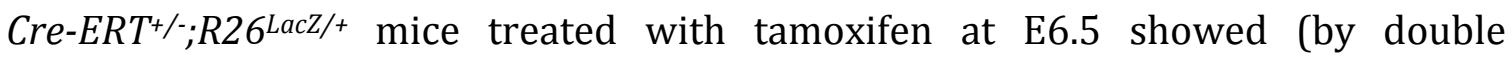
immunofluorescence) coincidence of $\beta$-gal+ cells derived from SCL+ precursors with SCL (red) and ACE (green). Scale bars: $50 \mu \mathrm{m}(\mathrm{A}) ; 10 \mu \mathrm{m}(\mathrm{B})$.
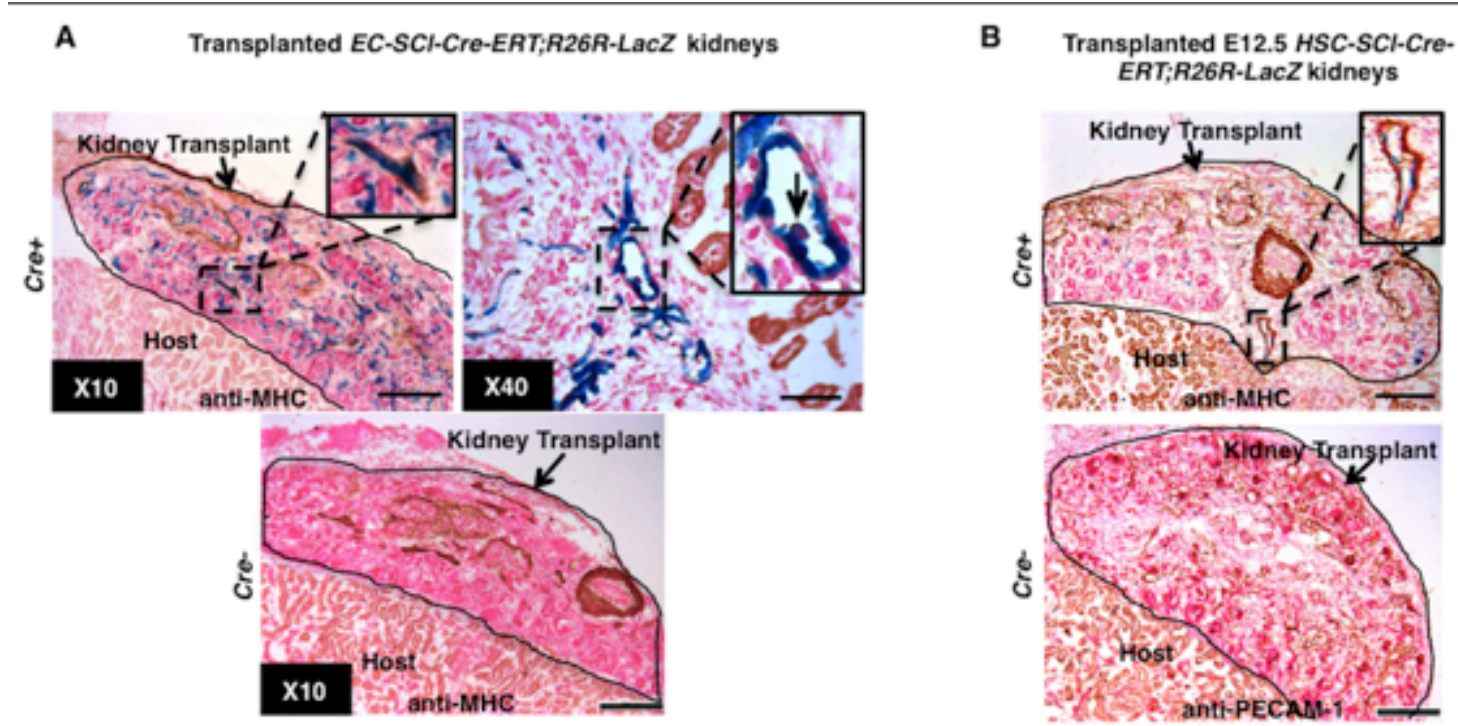

C

D
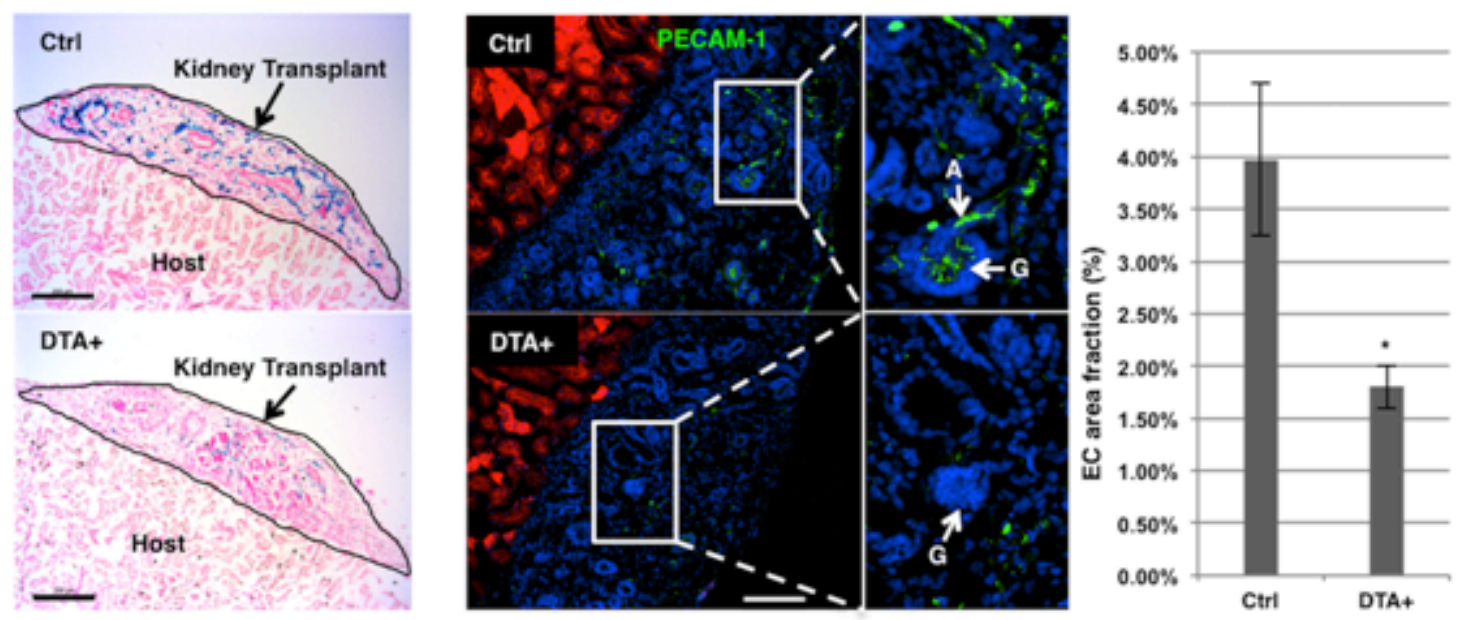

Figure 2.5. $S C L+$ precursors are required for hemo-vasculogenesis in the transplanted embryonic kidneys.

(A) EC-SCL-Cre-ERT ${ }^{+/ ;} 26^{\text {LacZ/+ }}(C r e+)$ prevascular embryonic kidney transplanted under the kidney capsule of an adult host for 7 days with tamoxifen treatment, developed $\beta$-gal+ vascular endothelium (in blue), with SMC coating, in brown (x10 
inset, anti-myosin heavy chain). Inset in x40 (right) shows a blood cell budding from an EC (arrow) in the transplanted embryonic kidney at higher magnification. The transplanted kidneys from Cre- control siblings (EC-SCL-Cre-ERT+/;R26 ${ }^{\mathrm{LacZ} /+}$ ) developed similar to the Cre+ ones after tamoxifen treatment, but were negative for X-gal staining. (B) Scattered $\beta$-gal+ cells in the developing vessels of transplanted

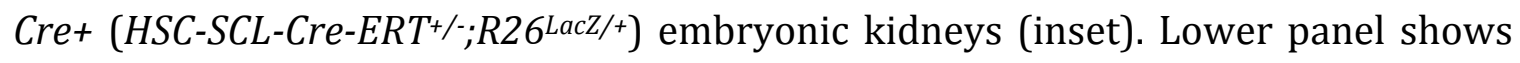
the Cre- (HSC-SCL-Cre-ERT-/;R26 LacZ/+) transplanted kidneys immunostained for PECAM-1. (C) Transplanted E12.5 DTA+ (EC-SCL-Cre-ERT+/;R26 LacZ/+;R26 ${ }^{\text {DTA/++})}$ and

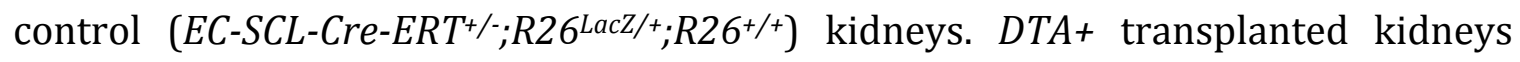
developed less $\beta$-gal+ ECs compared to their respective controls. (D) Immunofluorescence for PECAM-1 (green) in frozen sections from transplanted control (top panel) and DTA+ (bottom panel) kidneys. Nuclei were stained with hoechst (blue). Cells from host kidney express RFP (red). Inset of top panel shows at higher magnification the presence of well developed ECs of renal arterioles (A, arrow) and a normal glomerular capillary network (G, arrow) in a control transplanted kidney. Inset of bottom panel shows lack of ECs inside a glomerulus (G, arrow) in DTA+ transplanted kidneys. Note that ECs (green) in transplanted kidneys do not express RFP. Bar graph shows measurement of EC area fractions in frozen sections from transplanted control $(n=3)$ and DTA+ $(n=3)$ kidneys. Values are expressed as mean \pm SEM. * $\mathrm{p}<0.05$. Scale bars: $50 \mu \mathrm{m}(\mathrm{A}, \mathrm{x} 40)$; $200 \mu \mathrm{m}(\mathrm{A} \times 10, \mathrm{~B}-\mathrm{C})$; $100 \mu \mathrm{m}(\mathrm{D})$. 

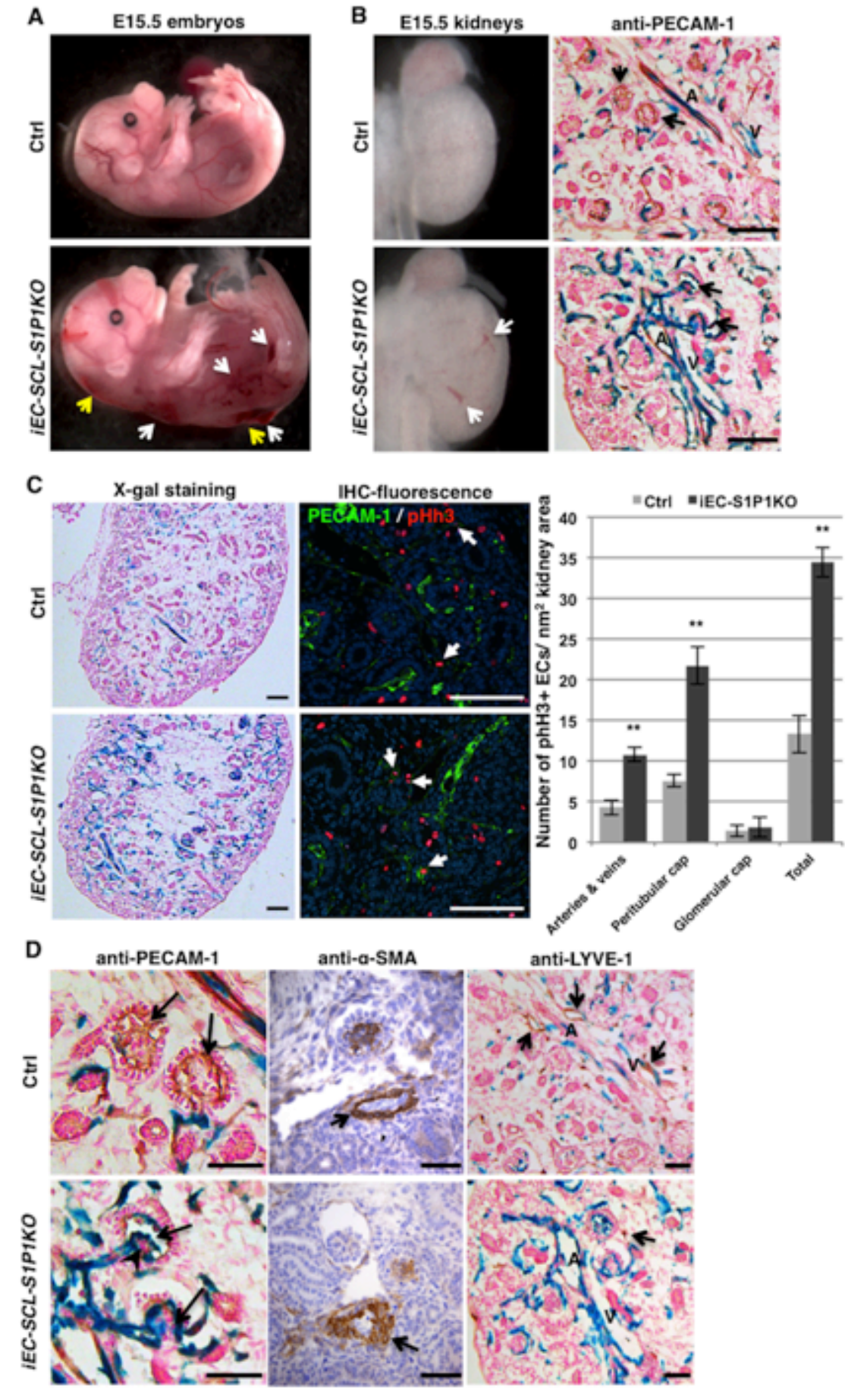

Figure 2.6. Timed deletion of S1P1 in endothelial precursors leads to abnormal renal vascular development. 
(A) Tamoxifen treated E15.5 iEC-SCL-S1P1KO (EC-SCLCre-ERT+/; S1P14/ff; R26 LacZ/++) embryos showed severe hemorrhages (white arrows) and dorsal subcutaneous edema (yellow arrows). However, the control siblings (EC-SCLCre-ERT $T^{+/ ;} S 1 P 1^{f /+}$; R26 $6^{\text {LacZ/+ }}$ ) were normal. (B) E15.5 cKO kidneys showed striking dilation of intrarenal arteries (A) and veins (V) evident in the whole kidney (Left, white arrows) and in histological sections subjected to X-gal staining and immunostaining for PECAM-1 (right). Black arrows indicate glomeruli. (C) X-gal staining of kidney sections showed hyperplastic endothelium labeled in blue in the cKO (left). Coimmunofluorescence staining for PECAM-1 (in green) and phosphorylated histone H3 (pHh3) (in red) shows proliferating ECs with coincidence of nuclear RFP with cell membrane GFP (arrows). Bar graph shows the number of proliferating ECs in different vessel types in E14.5 kidneys. The cKO kidneys show significant increase of EC proliferation in renal arteries, veins and peritubular capillaries (cap) but not in the glomerular capillaries (cap). Data are expressed as mean \pm SEM. ${ }^{* *} \mathrm{p}<0.01$. (D) Immunostaining for PECAM-1 showed that cKO kidneys lacked the normal capillary loops (upper panel, arrows), they developed capillary shunts (lower panel, arrows) accompanied by clumps of abnormal mensangial cells (arrowhead). Immunostaining for $\alpha$-SMA on kidney sections of control and cKO embryos showed abnormal arteriolar development with irregular smooth muscle coating in the cKO embryo (arrow). LYVE-1+ lymphatic vessels (upper panel, arrows) did not overlap with the $E C-S C L+$ derived cells (in blue) and were almost absent in cKO kidneys (lower panel, arrow points to a single LYVE-1+ cell). Scale bars: $100 \mu \mathrm{m}(\mathrm{B}-\mathrm{C}) ; 50 \mu \mathrm{m}$ (D). 


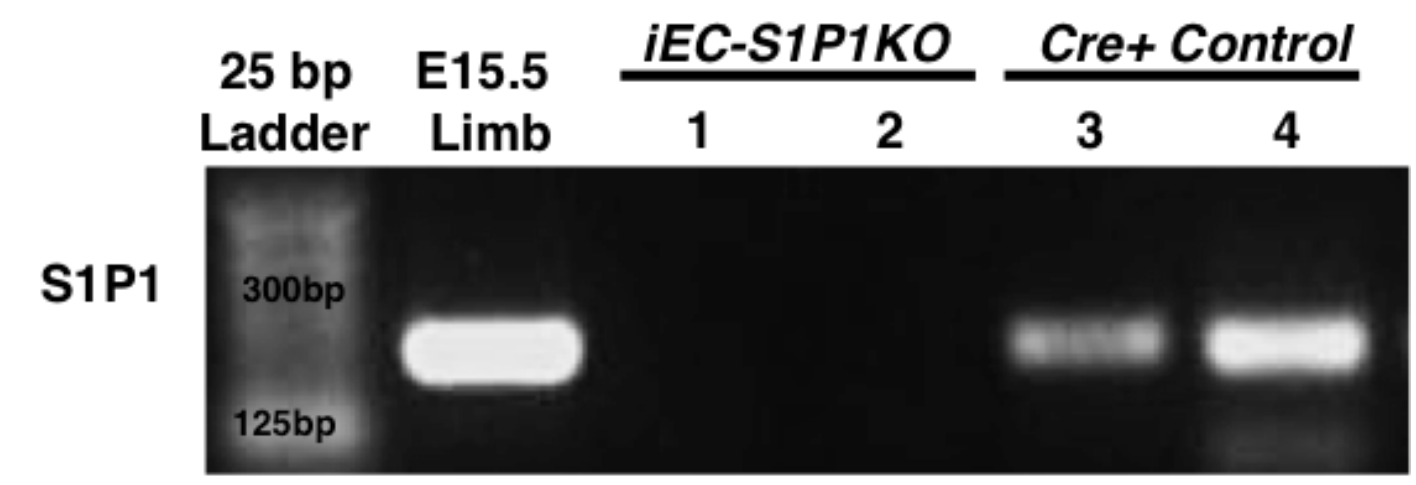

\section{GAPDH}

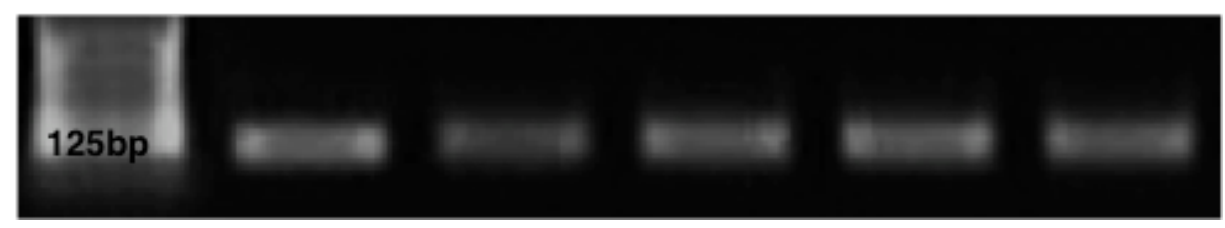

Figure 2.7. Deletion of S1P1 in SCL+ ECs from iEC-S1P1KO-mTmG mice.

RT-PCR for S1P1 and GAPDH from GFP+ cells sorted from kidneys of E15.5 iECS1P1KO-mTmG (EC-SCL-Cre-ERT $\left.T^{+/ ;} S 1 P 1^{\Delta / f l} ; R 26^{m T m G /+}\right)$ mice (1 and 2) and GFP+ cells sorted from kidneys of Cre+ control (EC-SCL-Cre-ERT+/; S1P14/+; R26 $6^{m T m G /+}$ ) siblings ( 3 and 4). The GFP+ cells from iEC-S1P1KO-mTmG mice show no expression of S1P1 whereas GFP+ cells from Cre+ control kidneys express S1P1. cDNA from E15.5 limb was used as a positive control. 

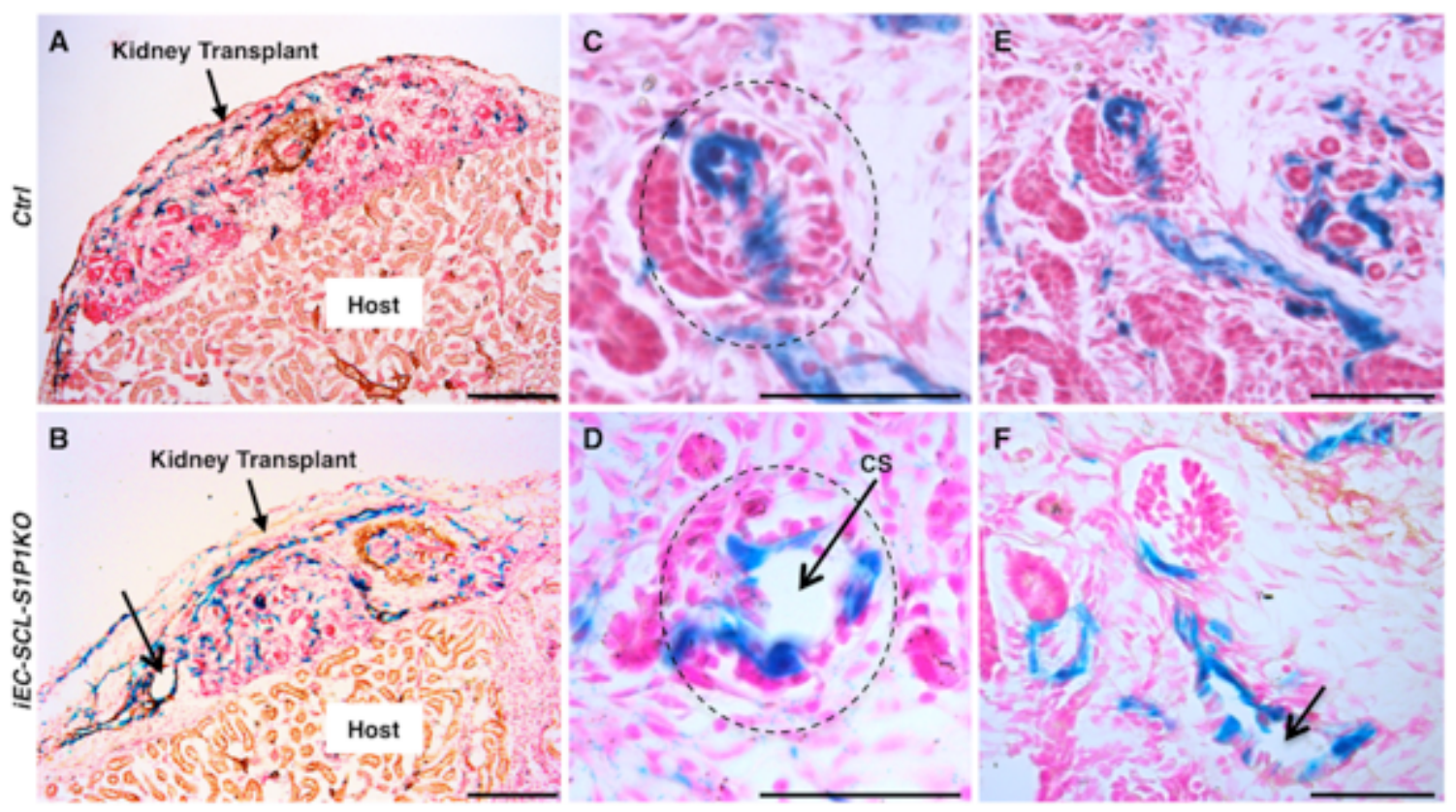

Figure 2.8. S1P1 functions intrinsically to regulate renal vascular development.

(A-B) X-gal staining and immunostaing for myosin heavy chain on sections from transplanted embryonic kidneys of control (A) and iEC-SCL-S1P1KO (B) mice showed increased number of $\beta$-gal+ ECs and dilated vessels (thin arrow) in the $i E C$ SCL-S1P1KO kidneys. (C-D) Control kidneys under transplantation showed normal development of glomerular capillaries (C, dotted circle) whereas iEC-SCL-S1P1KO kidneys developed capillary shunts (CS) (D, arrow). (E-F) Control kidneys under transplantation developed normal arterioles (E), whereas iEC-SCL-S1P1KO kidneys showed dilated arteriolar lumen (F, arrow). Scale bars: $200 \mu \mathrm{m}$ (A and B); $50 \mu \mathrm{m}$ (CF). 


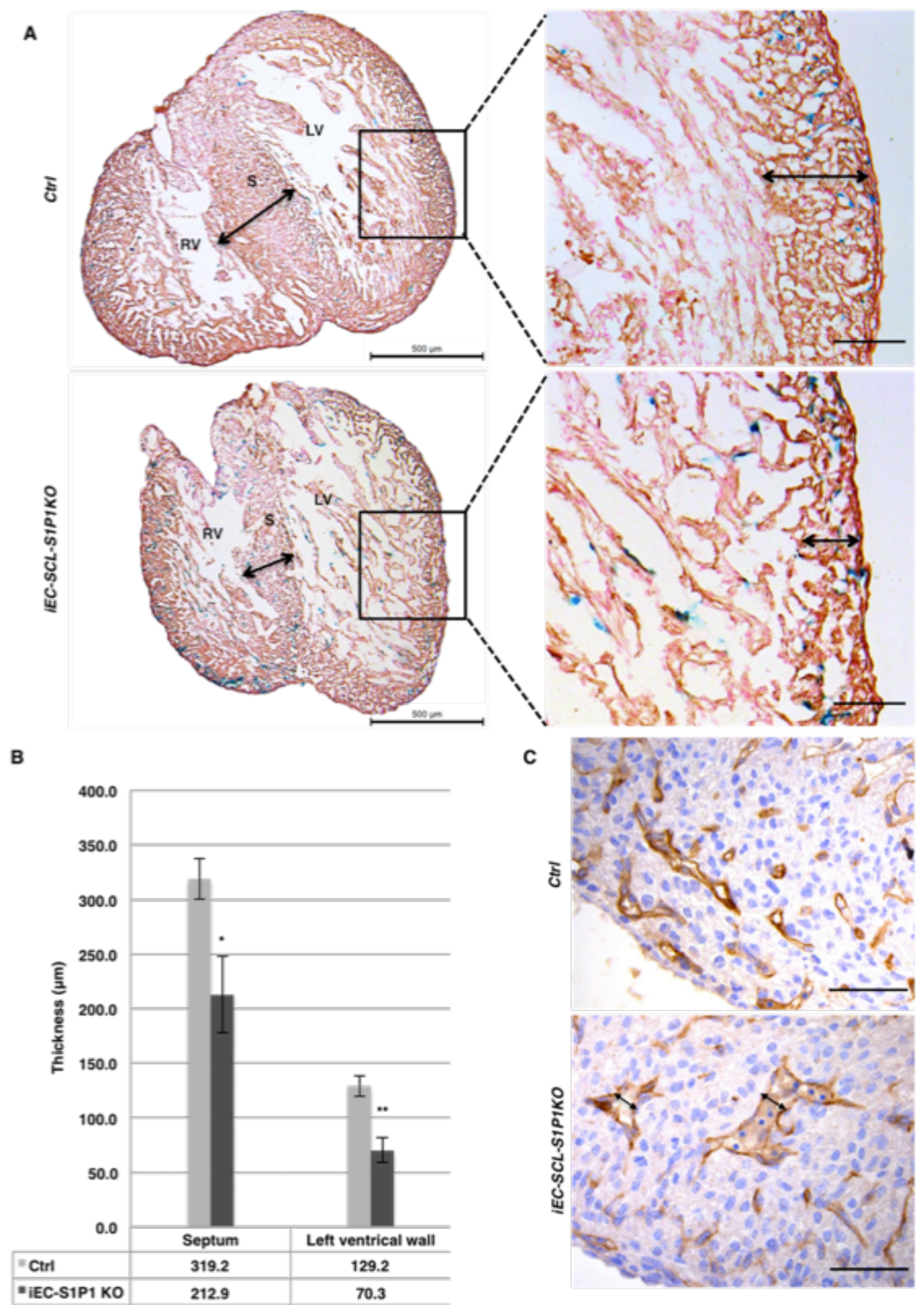


Figure 2.9. iEC-SCL-S1P1KO mice develop an abnormal heart. (A) X-gal staining and immunostaining for $\alpha$-SMA in longitudinal heart sections of control (Ctrl) and iEC-SCL-S1P1KO mice at E15.5. The iEC-SCL-S1P1KO mice developed thinner inter-ventricular septum (S) and inter-ventricular myocardium compact layer, especially in the left ventricle (LV). RV, right ventricle. (B) Thickness of septum and left ventricle wall of E15.5 control $(\mathrm{n}=4)$ and $i E C-S C L-S 1 P 1 K O(\mathrm{n}=4)$ mice. Values are expressed as mean \pm SEM. ${ }^{*} p<0.05,{ }^{* *} p<0.01$. (C) Immunostaining for PECAM-1 in heart sections of control and $i E C$-SCL-S1P1KO mice at E15.5 showing that the intra-myocardial vessels in the $i E C$-SCL-S1P1KO heart have larger diameter (double head arrows) than those in the control heart. Scale bars: $500 \mu \mathrm{m}(\mathrm{A}) ; 100 \mu \mathrm{m}$ (A, inset); $50 \mu \mathrm{m}(\mathrm{C})$. 


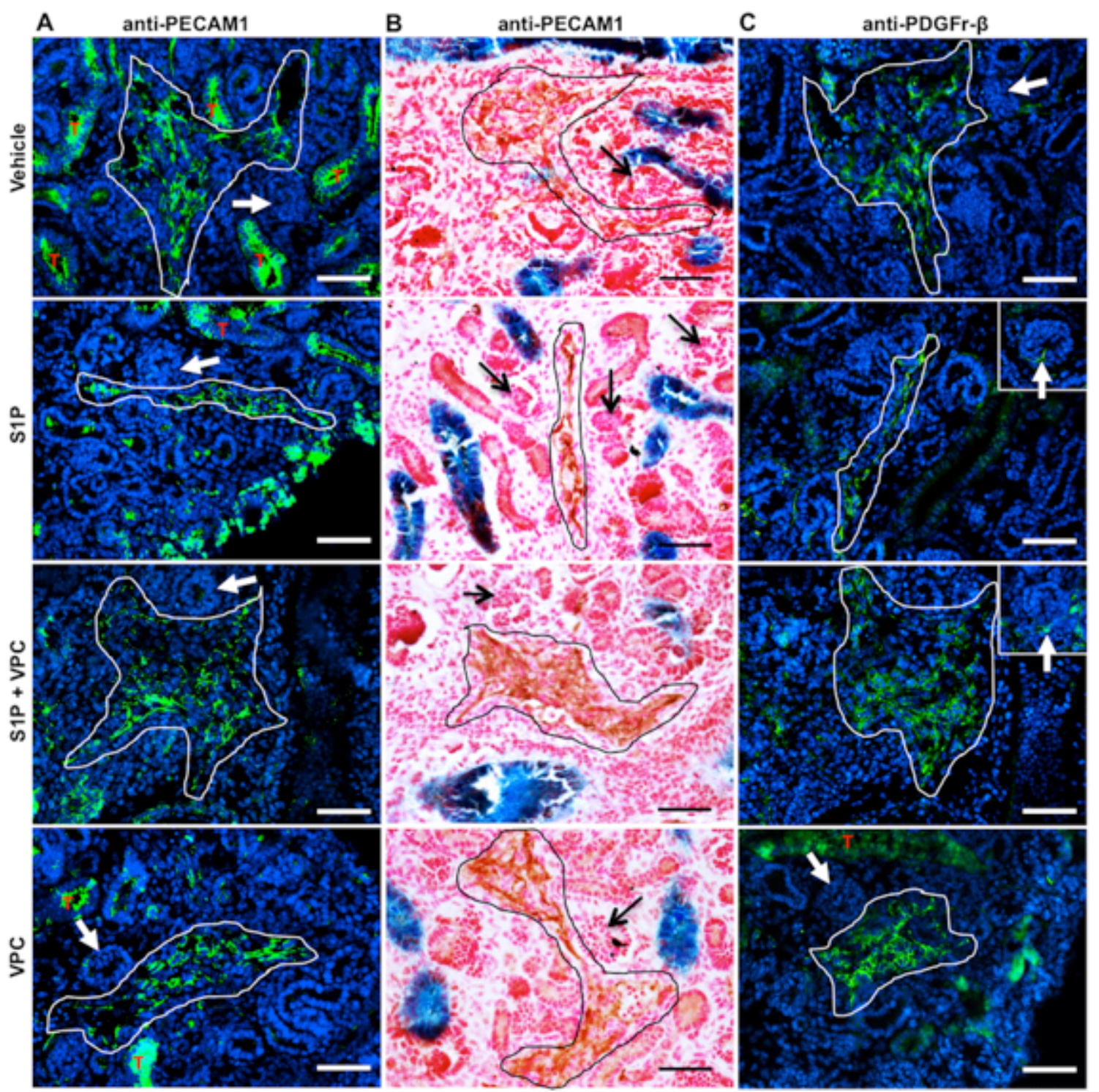

Figure 2.10. Ex vivo assessment of S1P-S1P1 signaling in the regulation of

\section{kidney vascular development.}

E12.5 Hoxb7Cre $/$; R26 $6^{\text {LacZ/+ }}$ kidneys treated with vehicle, S1P, S1P+VPC and VPC in culture for 72 hours and subjected to immunostaining for PECAM1 and PDGFr- $\beta$. (A) Immunofluorescence for PECAM-1 (in green) showed that control kidneys (vehicle) and kidneys treated with VPC developed thickened EC layer (white lined area), whereas those treated with S1P showed a thin layer of ECs. Kidneys treated with 
S1P + VPC developed even thicker EC layers in the renal vessels. Note that tubular ( $\mathrm{T}$, in red) staining in green was due to background signal (also found in control sections without the PECAM-1 antibody). (B) Similar results (without tubular background staining) were observed by immunohistochemistry using $\mathrm{DAB}$ as a substrate (black lined area). Glomerular capillaries did not develop in vitro (A and B, arrows). (C) Immunofluorescence for PDGFr- $\beta$ showed that a large number of PDGFr- $\beta+$ mural cells (white lined area) were closely associated with vessels in kidneys exposed to all treatments. Kidneys from all the groups lacked mesangial cells (arrows). Scale bar: $50 \mu \mathrm{m}$.

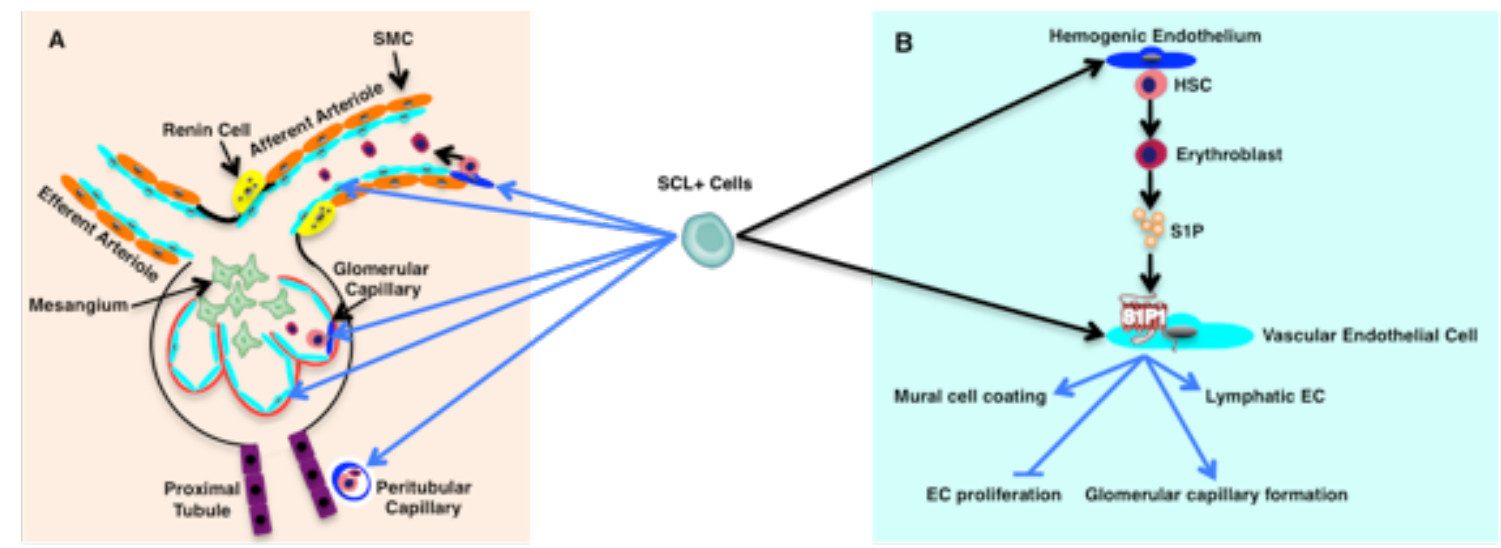

Figure 2.11. $S C L+$ precursors contribute to hemo-vasculogenesis in the kidney. Summary of findings on the lineage relationship between the SCL+ precursors and the cardiac endothelium, renal vascular endothelium and blood cells in the early embryo. Blue arrows indicate the lineage relationship from our results, while black arrows show published data from other groups. (A) The $S C L+$ precursor gives rise to definitive (light blue cells) and hemogenic (dark blue cells) vascular endothelium of renal vessels, including renal arteries, veins (not shown), glomerular capillaries and peritubular capillaries. (B) S1P-S1P1 signaling between blood cells 
and ECs derived from $S C L+$ precursors regulates kidney vascular development. Endothelial S1P1 is involved in mural cell coating of renal arteries and arterioles, development of glomerular capillaries and lymphatic ECs, and inhibits renal EC proliferation. 


\section{Chapter 3. Endocardium, coronary vascular endothelium and hematopoietic cells originate from hemo-vascular precursors and regulate heart development through sphingosin-1-phophate receptor 1}

Formatted as a first-authored manuscript to be submitted.

\section{Abstract}

The endocardium and coronary vascular endothelium have been suggested to originate from multiple sources, which also contribute to other cardiac cell types. Therefore, their embryonic origins and developmental mechanisms remain elusive. Specifically, the function of sphingosine-1-phosphate receptor 1 (S1P1) in early heart development is unclear, since it is ubiquitously expressed in the endothelium, myocardium and smooth muscle cells. Using fate tracing studies, immunohistochemistry, colony forming cells assays and embryonic heart transplantation, we identified an intermediate precursor population expressing stem cell leukemia $(S C L)$ gene driven by its $5^{\prime}$ end enhancer, which we termed hemo-vascular precursors. They exclusively give rise to the endocardium and coronary vascular endothelium, a subset of which possess hemogenic function during early cardiac development. Additionally, we demonstrated the essential role of endothelial S1P1 in the development of the myocardium and coronary vasculature. We showed that the deletion of S1P1 from the hemo-vascular precursors and their decedents in the heart led to ventricular non-compaction myopathy and coronary vascular abnormalities in the mouse embryos. In addition to helping understand the proper development of the cardiac endothelium and their 
alterations in the congenital heart diseases, our findings may provide information to heart regeneration therapies.

\section{Introduction}

During mouse embryogenesis, the earliest functional heart structure shows a linear tubular configuration consisting of an inner endocardial layer and an outer myocardial layer, which were suggested to derive from multiple progenitors. ${ }^{74}$ Lineage tracing studies revealed that a group of cardiac progenitor cells arise from the primitive streak mesoderm at embryonic day 7.5 (E7.5) and develop the first heart field (FHF), also known as the cardiac crescent, which at E8.5 forms the primitive heart tube, and subsequently the left ventricle and part of both atria. During elongation and looping of the heart tube, another group of tri-potential progenitor cells from the second heart field (SHF), are added to the arterial and venous poles, which differentiate into the endocardium, smooth muscle cells and myocardium of the right ventricle, outflow tract and part of both atria. ${ }^{73,74,128 \mathrm{~A}}$ recent study has identified the transcription cofactor homeodomain-only protein Homeobox (Hopx) as a marker of the FHF and SHF intermediate precursors committed to cardiomyocytes. ${ }^{77}$ However, the origin of cardiac endothelium remains intricate, precluding the precise functional analysis of genes expressed in the early endocardium and vascular endothelial cells (ECs).

In fact, the heart possesses two different types of endothelium: the endocardium, a structurally and functionally specialized endothelium, which may originate from both vascular endothelium and multipotent cardiac progenitors, ${ }^{129}$ and the 
coronary vascular endothelial cells (CVECs) that seem to develop from endocardium, sinus venous ECs and pro-epicardial precursors. 130, 131 Furthermore, previous studies have shown that blood islands were present in the sub-epicardium and out flow tract endothelium of the embryonic heart, ${ }^{132}$ and they were thought to derive in situ from hemogenic endothelium. ${ }^{133}$ Another study has shown that Nkx2.5+ endocardium contributes to transient definitive hematopoiesis in the early embryonic heart. ${ }^{41}$ Collectively, these data supports the notion that both endocardium and CVECs retain hemogenic function during early development and that the early embryonic heart may undergo hemo-vasculogenesis, the concomitant generation of blood and vessels. However, the origin of the hemogenic endocardium and CVECs is unknown.

As described in Chapter 2, the stem cell leukemia (SCL/Tal1) is a helix-loop-helix transcription factor required for the development of hemangioblasts, the common precursor of blood cells and endothelium. ${ }^{10,11}$ Its tissue specific enhancers direct gene expression in either endothelium or hematopoietic precursors. ${ }^{11}$ Fate tracing studies in zebra fish and expression analysis in mice demonstrated that the SCL is expressed in early endocardial precursors and regulate endocardial/myocardial specification and migration of endocardial cells. RW.ERROR - Unable to find reference:131 Using mice with tamoxifen inducible Cre expression driven by either enhancer, ${ }^{99,100}$ we recently identified an $S C L+$ hemo-vascular precursor giving rise to both vascular endothelium and blood cells in the early embryonic kidneys, which requires sphingosine-1-phophate receptor 1(S1P1) for the development of a normal vasculature. ${ }^{136}$ 
As described in Chapter 2, the sphingosine-1-phophate is a bioactive sphingolipid that regulates cell proliferation, migration and stabilization through activating five G protein coupled receptors (S1P1- S1P5). During heart development, S1P1 is predominantly expressed in the endocardium, cardiomyocytes and vascular ECs from E8.5, before it is expressed in the vasculature of the head and body which starts at E10.5. 114, 137, 138 Global knockout of S1P1 in the mouse leads to early embryonic lethality with abnormal vasculature and impaired heart development, which showed reduced proliferation of myocardial cells and abnormal fibronectin expression in the subepicardium and the atrioventricular (AV) cushion. ${ }^{137}$ Since numerous cardiac cell types respond to the S1P-S1P1 singling pathway, the role of S1P1 in each cell type needs to be assessed. Interestingly, while we study the role of S1P1 in the kidney vascular development, we found that deletion of S1P1 from the $S C L+$ precursors led to bradycardia, non-compaction of the left ventricle, thin ventricular septum and dilated coronary vessels, ${ }^{136}$ suggesting an indispensable role of S1P1 during heart development. However, the contribution of SCL+ precursors to the heart and the role of endothelial S1P1 in heart development have not been previously investigated.

Here, we showed that the SCL expression driven by its 5' end endothelial enhancer ( $S C L^{\mathrm{EC}}$ ) labels cardiac hemo-vascular precursors that give rise to both endocardium and CVECs, a subset of which possessed hemogenic potential. Partial and complete ablation of these precursors led to growth retardation, cardiac hypotrophy and loss of endocardium. Further, we induced early deletion (E7.5) of S1P1 from $S C L^{E C_{+}}$ precursors and their progeny in mice, which developed non-compaction of both 
ventricles and abnormal coronary vessels, suggesting an indispensable role of endothelial S1P1 for heart development.

\section{Results}

3.1 $S C L^{E C}+$ enhancer driven reporter expression marks cardiac precursors committed to endothelial fate.

To label the $S C L^{E C_{+}}$precursors in the developing heart, we first crossed $E C$-SCL-Cre$E R T^{+/-}$mice to $R 26^{m T m G / m T m G}$ mice to obtain EC-SCL-Cre-ERT $T^{+/ ;}$R26 $6^{m T m G /-}$ embryos, and performed a single maternal injection of tamoxifen to pregnant mice at embryonic day 7.5 (E7.5), when the cardiac crescent starts to form. We performed histological analysis of embryos from E9.5 to E13.5 as shown in Figure 3.1A. At E9.5, GFP reporter expression labeled the endocardium of the outflow tract, ventricles and atria but not the myocardium (Figure 3.1B and $\mathrm{C}$ ). In addition to the endocardium, at E10.5 GFP also labeled the atrioventricular cushion (AVC), which may undergo endothelial-mesenchymal transformation to form future valves and septae (Figure 3.1D and E). From E12.5 to E13.5, GFP expression expanded in the developing endocardium, AVC and coronary vascular endothelial cells (CVECs), and appeared within the myocardium, ventricular septum and sub-epicardium. (Figure $3.1 \mathrm{~F}$ and $\mathrm{G})$

Further, double immunofluorescence staining for GFP and the endothelial marker PECAM-1 demonstrated that the endocardium (Figure 3.1G) and the majority of CVEC (Figure 3.1J and K) co-expressed GFP and PECAM-1. Notably, a small population of CVECs and most of the aortic ECs were not labeled by GFP expression 
(Figure $3.1 \mathrm{H}$ and $\mathrm{K}$ ), which may be due to either missing tamoxifen induction or a distinct origin from non-SCLEC+ precursors. Additionally, we identified GFP+ PECAM- CVECs, which form vascular plexus in the myocardium (Figure 3.1J) and blood-islands co-expressing GFP and hemoglobin ( $\mathrm{Hb}$ ) in the atrioventricular groove (Figure 3.1K and L), suggesting immaturity and potentially high mobility of these cells. In summary, the $S C L^{E C}+$ precursors give rise to the endocardium, AVC and the majority of CVECs.

3.2. Fate tracing of $S C L^{E C_{+}}$precursors identifies hemo-vasculogenesis in early embryonic hearts.

To evaluate whether in the developing heart $S C L^{E C_{+}}$precursors possess hemogenic properties we performed the following series of experiments. Immunofluorescence for GFP, Runx1 (a master regulator of blood formation) and $\mathrm{Hb}$ in heart sections of EC-SCL-Cre-ERTCre/-;R26 $6^{m T m G /-}$ mice (following the scheme in Figure 3.1A) showed at E9.5 and E10.5 cells co-expressing GFP and Runx1 or $\mathrm{Hb}$ in the endocardium of the outflow tract (OFT), ventricles and atria (Figure 3.2A-D). At E12.5 we observed $\mathrm{GFP}+/$ Runx1+ blood islands in the ventricular septum (Figure 3.2E) and GFP+/Hb+ cells in ventricular endocardium (Figure 3.2F). These data suggest that the $S C L^{E C_{+}}$ precursors give rise to hemogenic endocardium and blood islands within the developing embryonic heart.

To further assess the hemogenic capacity of the early $S C L^{E C}$ precursors in the embryonic heart, we performed methylcellulose-based colony forming cell (CFC) assays. We harvested cells from the hearts and different embryonic areas including 
major hematopoietic organs (yolk sac and the caudal half at E9.5, the caudal half at E10.5 and fetal liver at E12.5) at different embryonic stages from EC-SCL-Cre-ERT ${ }^{+/}$ ;R26 $6^{\mathrm{mTmG} /-}$ mice with tamoxifen treatment at E7.5 (Figure 3.2G). Two types of early stage hematopoietic colonies: CFU-GEMM (granulocytes, erythrocytes, monocytes/macrophages and megakaryocytes) and CFU-GM (granulocytes and macrophages) formed not only from hematopoietic organs but also from the cells of the hearts. The CFU-Mix (GEMM and GM) colonies formed at a rate of $2.3( \pm 1)$ per 1000 heart cells with 43\% of the colonies expressing GFP when cultured from E9.5 hearts (Figure 3.2H). The number of colonies reached a peak when cultured from E10.5 hearts (8.4 \pm 0.6 colonies per 1000 cells, 50\% GFP+) (Figure 3.2I). By E12.5, the number of CFU-Mix colonies derived from heart cells decreased to $1.3( \pm 0.3)$ per 1000 cells with 8.4\% GFP+ colonies (Figure 3.2J). Overall, these results confirmed that the $S C L^{E C_{+}}$precursors give rise to early hematopoietic precursors within the early embryonic heart.

3.3. Ablation of $S C L^{E C}+$ precursors impair hemo-vasculogenesis and heart development.

To investigate whether $S C L^{E C}$ precursors were indispensable for the normal development of the heart we designed the following ablation experiment. We crossed $R 26^{D T A / D T A}$ mice with $E C$-SCL-Cre-ERT//;R26R- $m T m G^{+/-}$mice to generate $E C$ SCL-Cre-ERT $T^{+/ ; R 26^{D T A /+} ; R 26^{m T m G /+}(D T A+)}$ mice, which express diphtheria toxin subunit A (DTA) and GFP in the $S C L^{E C_{+}}$precursors. All the DTA+ embryos died within $24 \mathrm{hrs}$ with a full dose of tamoxifen injection (1mg/30g bodyweight) at E7.5, whereas their $\mathrm{Cre}+\left(E C-S C L-C r e-E R T^{\left.+/-; R 26^{m T m G /+}\right)}\right.$ control littermates were normal (data not shown). When we injected pregnant mice with half the dose of tamoxifen 
$\left(0.5 \mathrm{mg} / 30 \mathrm{~g}\right.$ bodyweight) to partially ablate the $S C L^{E C_{+}}$precursors, the DTA+ embryos survived to E9.5, however showed growth retardation (Figure 3.3A and B). Immunofluorescence for GFP and the myocardial marker cardiac muscle troponin $\mathrm{T}$ (cTnT) in heart sections of control embryos further confirmed that part of the $S C L^{E C_{+}}$lineage endocardium was still labeled by GFP+ expression, whereas the DTA+ embryos almost completely lost $S C L^{E C_{+}}$lineage endocardium labeled with GFP. Additionally, the myocardium of DTA+ embryos had impaired trabeculation. (Figure 3.3C and D)

Because the $S C L^{E C}$ precursors contribute to the vascular system all over the embryo, the heart developmental abnormities in the $D T A+$ embryos may be influenced by the systemic vascular abnormalities. To investigate whether ablation of $S C L^{E C_{+}}$ precursors affects cardiac development intrinsically, we developed an in vivo crosstransplantation model ${ }^{136}$, which allows the development of the embryonic heart under the kidney capsule of the host mice and the visualization of a beating heart at the time of harvest. We transplanted hearts of E9.5 DTA+ mice and their control siblings under the kidney capsule of wild type adult host mice and induced DTA and GFP expression in $S C L^{E C_{+}}$precursors after transplantation. Immunofluorescence staining for GFP and cTnT showed that the GFP+ endocardium developed within the heart of the control transplants, with $\mathrm{GFP}+\mathrm{Hb}+$ blood islands (Figure 3.3F and $\mathrm{H}-\mathrm{J}$ ), which further confirmed the contribution of $S C L^{E C_{+}}$precursors to intrinsic hemovasculogenesis in the heart. However, the transplanted hearts from DTA+ mice completely lost the GFP+ endocardium and the heart lumen structure (Figure 3.3G). 
These experiments underscored the requirement of $S C L^{E C_{+}}$precursors within the early embryonic heart for hemo-vasculogenesis and organogenesis.

\subsection{S1P1 expressed in SCLEC lineage endothelium regulates heart development}

To investigate the role of endothelial S1P1 during early cardiac development, we designed a series of experiments to deletion S1P1 from the $S C L^{E C_{+}}$precursors during heart development. We treated $C E C-S C L-S 1 P 1 K O$ mice and their control siblings with tamoxifen at E7.5 to induce GFP reporter expression and/or S1P1 deletion in the $S C L E C+$ lineage cells. The $c E C-S C L-S 1 P 1 K O$ and control embryos died in utero at E13.5 to E14.5, which may have been due to tamoxifen toxicity. Before E13.5, the littermate controls of the $c E C-S C L-S 1 P 1 K O$ embryos were morphologically normal, indistinguishable from the non-injected ones. Therefore, we characterized the hearts of $c E C-S C L-S 1 P 1 K O$ mice from E12.5 to E13.5.

The $c E C$-SCL-S1P1KO mice developed thin ventricular walls and thick trabeculae. (Figure 3.4A-D) To study whether proliferation of the myocardium was affected, we performed co-immunofluorescence staining for phosphorylated histone H3 (phh3) and cTnT in heart sections of E12.5 $c E C-S C L-S 1 P 1 K O$ and control mice. The results demonstrated a remarkable decrease in the proliferation of the myocardium in both ventricles and atria of the $c E C$-SCL-S1P1KO mice. (Figure 3.4E) However, there was no significant difference in the trabeculae (Figure 3.4E)

Interestingly, we found that $c E C-S C L-S 1 P 1 K O$ embryos developed less peri-truncal CVECs within the ventricular wall than the controls (Figure 3.34C, D and F), whereas their CVECs in the septum were increased (Figure 3.4G). We did not find a difference 
in the number of ECs within the endocardium or AVC between the $c E C-S C L-S 1 P 1 K O$ and control embryos. (Figure 3.4G)

\section{Discussion}

In this study we show that the expression of the $S C L$ gene driven by its 5' endothelial enhancer marks cardiac precursors committed to an endothelial fate, which give rise to endocardium and CVECs, a subset of which undergo hemo-vasculogenesis to generate ECs and blood cells. Genetic ablation of these precursors in vivo or after transplantations resulted in absence of endocardium and subsequent abnormal myocardial development. Further, using this marker gene, we were able to specifically delete $S 1 P 1$ from cardiac endothelium during early heart development, which demonstrated essential roles of endothelial S1P1 for the development of ventricular and atrial cardiomyocytes and coronary vascular formation.

Collective data from lineage analysis suggested that the endocardium, CVECs and vascular endothelium elsewhere are highly related in cellular origin. For instance, fate-tracing studies of Nfatc1+ endocardium demonstrated that the endocardium is a major source of the arterial CVECs through angiogenesis. ${ }^{131}$ Albeit the majority of venous CVECs and a small number of arterial CVECs were derived from the dedifferentiated sinus venous endothelium. ${ }^{139}$ Additionally, another study has identified a subset of pro-epicardial precursors giving rise to a small population of CVECs and smooth muscle cells. ${ }^{130}$ However, none of the genes used in these studies exclusively labeled the whole population of cardiac ECs, including endocardium and CVECs. The lack of specific marker genes for intermediate precursors committed to 
the cardiac endothelial fate has hampered our understanding of the molecular basis of cell fate decision and cell differentiation in the heart. Here, we report that timely labeled early $S C L^{E C_{+}}$precursors gave rise to all the endocardium and the majority of CVECs during heart development.

Our studies identified intrinsic $S C L^{E C_{+}}$derived hemogenic ECs and early hematopoietic precursors in E9.5-E12.5 hearts and hematopoietic organs by immunostaining, CFC assay and cross-transplantation studies. Although, cell surface marker expression studies in embryonic stem cells suggested that cardiac progenitors and hemangioblasts were two distinct populations, ${ }^{76,140-142}$ our data suggest a contribution of cardiac hemo-vascular precursors to the hemogenic endocardium and CVECs. Moreover, we detected Runx1 protein by immunostaining, the marker of early hematopoietic stem cells, ${ }^{143}$ in the endocardium and CVECs of early embryonic heart, which is in agreement with the RT-PCR data from others. ${ }^{144}$ These results further confirmed a hemogenic function of the early cardiac endothelium. In our CFC studies, fewer colonies were found from the E12.5 heart than from earlier stages. This may due to low percentage of endothelium in the whole heart, since the cardiomyocytes and other types of cells proliferate quickly and account for a large proportion of the total heart cells at this age. Further, the lower percentage of GFP expressing colonies from the E12.5 heart suggested a decreased contribution of $S C L^{E C}$ precursors to the cardiac hematopoiesis, which is in agreement with the transient hemogenic function of the embryonic heart previously described. ${ }^{41}$ The unlabeled colonies may differentiate from hematopoietic 
precursors that were generated earlier than the tamoxifen treatment or may have escaped the induction with tamoxifen.

Furthermore, our $C E C$-SCL-S1P1KO mice developed thin ventricular myocardium accompanied with abnormal trabeculation and coronary vascular development, which mimics the ventricular noncompaction cardiomyopathy observed in human patients. These patients develop hypertrabeculation with deep recesses in thin ventricular walls, which are usually accompanied with coronary vascular diseases and arrhythmias. ${ }^{145}$ Similar to the S1P1 global knockout mice, our $c E C-S C L-S 1 P 1 K O$ mice showed reduced myocardial proliferation in the ventricles and atria, suggesting that myocardial development is regulated by endothelial S1P1 in a nonautonomous manner. Whether S1P1 plays an intrinsic role in the myocardium, remains to be investigated. Although we found thickened trabecular tissue in the heart of $c E C$-SCL-S1P1KO mice, their proliferation was not significantly increased. It has been reported that the trabeculation is regulated by growth factors secreted by the endocardium, such as neuregulin growth factors. ${ }^{146,} 147$ Therefore, deletion of S1P1 from endocardium may affect expressions of these factors and consequently result in abnormal trabeculae. Notably, the coronary vessels in the ventricle wall were reduced in response to deletion of endothelial S1P1, whereas those in the septum were increased. It suggests a potentially distinct role of S1P1 during their development. It has been reported that the cross-talk between the fibroblast growth factor (FGF) signaling from the epicardium and the endothelial growth factor A (VEGF-A) signaling from the myocardium regulates the development of ventricular CVECs. ${ }^{148}$ However, the septal CVECs are only affected by the deletion of VEGF-A 
from the myocardium but not the deletion of VEGF-C from the epicardium. ${ }^{131,149}$ How the endothelial S1P1 interacts with these signaling pathways to regulate coronary vascular development in the ventricles and septum remains to be investigated.

In summary, our study has identified a specialized $S C L^{E C}$ precursor exclusively committed to a cardiac endothelial fate, which gives rise to the endocardium and CVECs, including a subset with hemogenic potential. Our mouse model will enable the isolation of early cardiac EC precursors for the analysis of genetic networks underlying the cardiac development, and will ultimately be of help in the heart regeneration field. Furthermore, by deleting S1P1 from the $S C L^{E C_{+}}$precursors and their derivatives, we uncovered the unique role of endothelial S1P1 in the myocardial and coronary vascular development. These findings may help advance our understanding of genetic defects that trigger congenital heart disease and development of new treatment for the patients.

\section{Methods}

\subsection{Animals}

For fate tracing studies, colony-forming cell assays and embryonic heart transplantations, EC-SCL-Cre-ERT mice 99 were crossed to B6.129(Cg)Gt(ROSA)26Sortm4(ACTB-tdTomato,-EGFP)Luo $/ \mathrm{J}\left(R 26^{m T m G / m T m G}\right) 123$ reporter mice. Pregnant mice were injected with $(1 \mathrm{mg} / 30 \mathrm{~g}$ body weight $)$ tamoxifen intraperitoneally. To genetically ablate $S C L^{E C+}$ precursors, $E C$-SCL-Cre-ERT mice were crossed to B6.129GT(ROSA)26SSortm1(DTA)Lky $/ \mathrm{J}$ mice $\left(R 26^{\text {DTA/DTA }}\right)^{125}$ and $R 26^{m T m G / m T m G}$ mice. Partial 
ablation of $S C L^{E C_{+}}$precursors was achieved by injecting pregnant mice with half dose of tamoxifen $(0.5 \mathrm{mg} / 30 \mathrm{~g}$ body weight). To study the role of S1P1 expressed by cells from the $S C L^{E C_{+}}$lineage during heart development, $C E C$-SCL-S1P1KO mice were generated as previously described. ${ }^{136}$ All procedures were performed in accordance with the Guiding Principles for Research Involving Animals and Human Beings by the American Physiological Society and were approved by the University of Virginia Animal Care Committee.

\subsection{Whole-mount organ imaging and immunofluorescence}

For whole-mount heart imaging, the hearts from EC-SCL-Cre-ERT+/;R26 $6^{m T m G /-}$ mice at E9.5 $(n=3)$, E10.5 $(n=4)$ and E12.5 $(n=4)$ were micro-dissected and fixed in $4 \%$ paraformaldehyde for $5 \mathrm{~min}$ and placed in cold PBS. Images were captured using a Q Imaging RETIGA EXi camera connected to a Leica DMIRE2 microscope.

Immunofluorescence was performed on $5 \mu \mathrm{m}$ sections of Bouins fixed and paraffin embedded tissues following standard protocols. ${ }^{127}$ Primary antibodies used were anti-GFP (1:500 dilution, Abcam, ab13970), anti-PECAM-1 (1:500 dilution, Santa Cruz, sc1506-R), anti-phh3 (1:200 dilution, Cell Signaling, \#9701S), anti-cTnT (1:200 dilution, Abcam, ab8295), anti-Hb (1:500 dilution, DAKO, A0118) and antiRunx1 (1:500 dilution, Abcam, ab23980). The secondary antibodies used were Alexa Fluor 488 goat anti-chicken IgG (H+L) (GFP), Alexa Fluor 568 donkey anti-rabbit IgG (H+L) (PECAM-1, phh3, Hb and Runx1) and Alexa Fluor 568 donkey anti-mouse IgG $(\mathrm{H}+\mathrm{L})$ (1:500 dilution; Life Technologies, Carlsbad, CA). Nuclei were stained with Hoechst 33342 (1:1000dilution, Invitrogen, H3570) Images were obtained using a 
Leica DFC360 FX digital camera connected to a Leica DFC 480 microscope.

\subsection{Colony-forming-cell assays}

Hearts [at E9.5 $(n=3)$, E10.5 $(n=4)$ and E12.5 $(n=5)]$, caudal halves [at E9.5 $(n=3)$ and E10.5 (n=4)], yolk sacs (at E9.5, $\mathrm{n}=3$ ) and fetal livers (at E12.5, $\mathrm{n}=5$ ) from $E C$ -

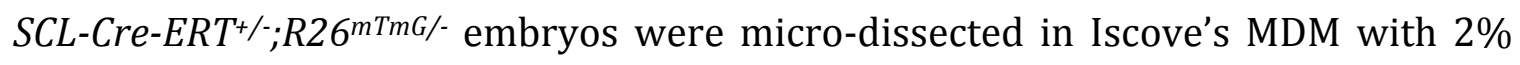
FBS and disaggregated with collagenase (Stemcell technologies, M7902) at $37^{\circ} \mathrm{C}$. The dissociated single cells were plated in $1 \mathrm{ml}$ of the Methocult GF (M3434, StemCell Technlogies) and cultured in 24 well cell culture plates at $37^{\circ} \mathrm{C}$ in $5 \%$ humidified $\mathrm{CO}_{2}$. After 12 days of incubation, the colonies were scored as described previously. ${ }^{136}$

\subsection{Embryonic heart transplantation}

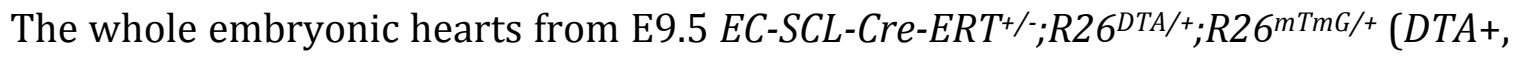
$\mathrm{n}=3)$ mice and their control siblings ( $\left.E C-S C L-C r e-E R T^{+/} ; R 26^{m T m G /+}, \mathrm{n}=3\right)$ were microdissected and transplanted under the kidney capsule of host mice. Briefly, after the host mice were anesthetized with tribromoethanol ( $0.25 \mathrm{mg} / \mathrm{g}$ body weight), an incision on the kidneys capsule was made using a Vannas scissor. A 27-gauge needle was inserted into the incision to carefully extend the space between the capsule and the host kidney. Then the freshly micro-dissected embryonic hearts were placed under the kidney capsule of the host mice. After surgeries, the host mice were injected with analgesic drug buprenorphine $(0.2 \mathrm{mg} / \mathrm{kg}$ body weight $)$ intraperitoneally daily for 2 days and tamoxifen $(1 \mathrm{mg} / 30 \mathrm{~g}$ body weight) 
intraperitoneally daily for 7 days as previously described for another embryonic organ. 136

\subsection{Measurement of myocardium proliferation}

To measure the number of proliferating myocardial cells, we performed doubleimmunofluorescences for phh3 and the myocardial marker cTnT in 5 comparable sections from the heart of E12.5 $c E C-S C L-S 1 P 1 K O(n=4)$ and control $(n=4)$ embryos. The number of phh+/cTnT+ cells was counted from each cardiac structure (ventricles, atria, septum, AVC and trabeculae). The areas of each cTnT+ cardiac structure were measured by imageJ.

5.6 Measurement of areas of endocardium and coronary vascular endothelial cells (CVECS)

To measure the area of PECAM-1+ endocardium in the ventricles and atria and CVEC in the septum, we performed immunofluorescences for PECAM-1 and cTnT in 5 comparable sections from the heart of E12.5 $c E C-S C L-S 1 P 1 K O(\mathrm{n}=4)$ and control $(n=4)$ embryos. The PECAM-1+ areas and cTnT+ areas were measured using imageJ, respectively. The values were expressed as percentages:

$$
E C \text { area }(\%)=\frac{P E C A M-1+\text { area }}{c T n T+\text { area of each cardiac structure }}
$$

To measure the area of GFP+ CVECs in the ventricles, we performed doubleimmunofluorescences for GFP and cTnT in 5 comparable sections from the heart of E12.5 $c E C-S C L-S 1 P 1 K O(n=5)$ and control $(n=5)$ embryos. The GFP+ ventricular CVEC areas and the total ventricular area were measured using imageJ. The areas of 
GFP+ endocardium and septal CVECs were manually excluded. The values were expressed as percentages:

$$
\text { ventricular CVEC area }(\%)=\frac{G F P+\text { ventricular } C V E C \text { area }}{\text { Total ventricular area }}
$$

\subsection{Statistical analysis}

Data are shown as mean \pm SEM. Statistical analysis was carried out by Student's ttest. A p $<0.05$ was considered significant. 


\section{Figures and figure legends}
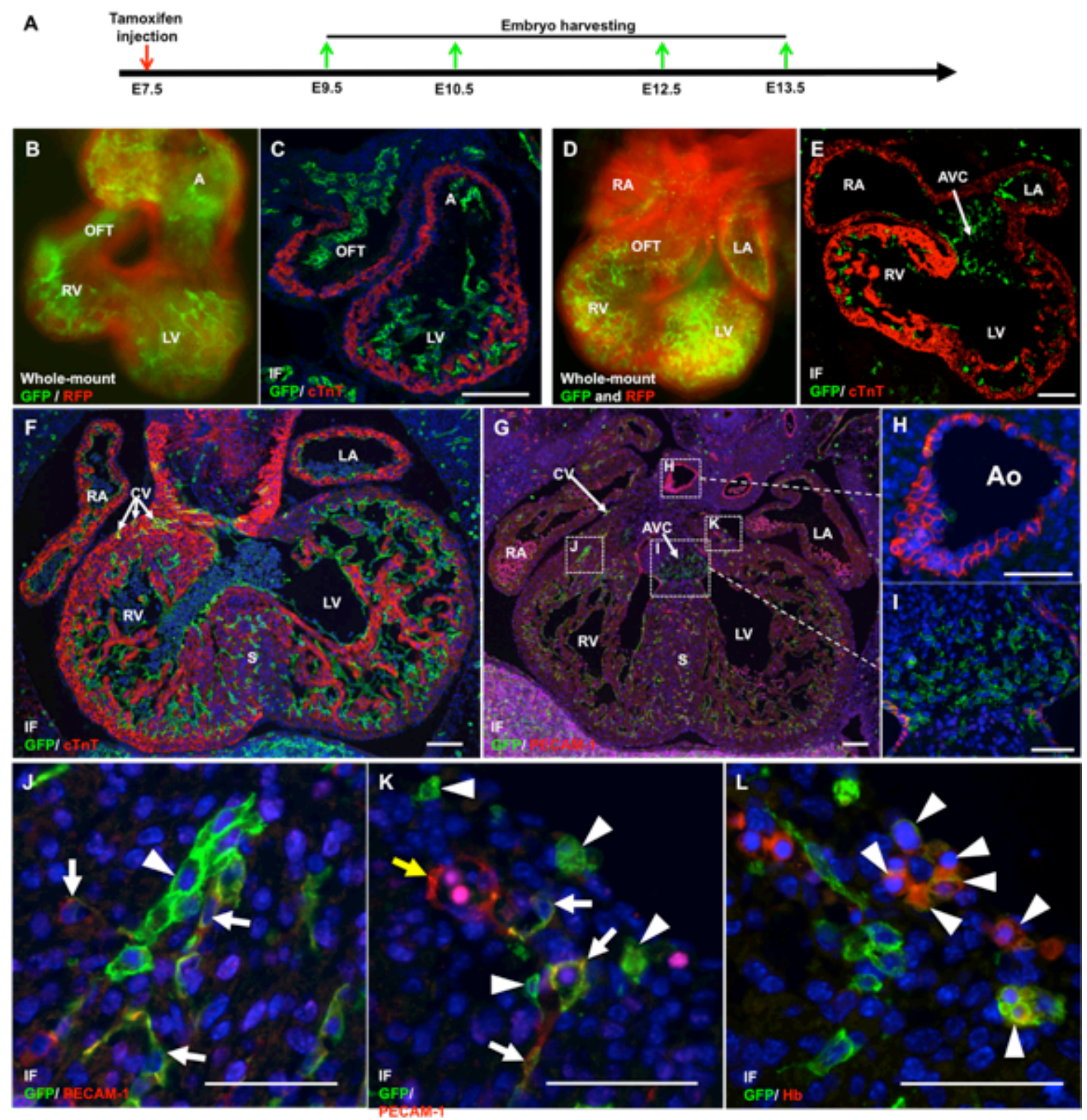

Figure 3.1. $S C L^{E C}+$ precursors differentiate into endothelium and endocardium.

[A] To trace the fate of $S C L^{E C}+$ precursors, EC-SCL-Cre-ERT $T^{+/} ; R 26^{m T m G /-}$ mice were treated with tamoxifen at E7.5 by maternal injection (red arrow) Embryos were harvested at E9.5, E10.5 , E12.5 and E13.5 (green arrows). 
[B-E] Whole-mount fluorescent images (B and D) and immunofluorescence (IF) for GFP (green) and cTnT (red) in heart sections (C and E) show that the $S C L^{E C_{+}}$ precursors give rise to endocardium but not myocardium in both ventricles, atria and outflow tract (OFT) of the heart tube at E9.5 (B and C) and the endocardium in the four chambers, atrial-ventricular cushion (AVC) and OFT of the heart at E10.5 (D and E).

[F] In addition to endocardium, the $S C L^{E C+}$ precursors give rise to coronary vascular (CV) endothelial cells in the atrial-ventricular groove and in the septum of E12.5 heart. [G-I] IF for GFP and PECAM-1 in E13.5 heart sections showed that GFP was expressed in all the PECAM-1+ endocardium in the four heart chambers $(\mathrm{G})$ and PECAM-1- AVC (I), but not in aortic endothelial cells (ECs) (H).

[J-K] Most of the CV ECs in the E13.5 heart sections co-expressed GFP and PECAM-1 (arrows). Arrowheads indicate a sub population of GFP+/PECAM-1+ ECs forming vascular plexus (J) and blood island-like structures (K). Only a few GFP-/PECAM-1+ CV ECs were identified. (yellow arrow).

[L] IF showed that the blood island-like structures were GFP+/Hb+ (arrowheads).

A, atria; LV, left ventricle; RV, right ventricle; OFT, outflow tract; AVC, atrialventricular cushion; CV, coronary vessel; Ao, aorta; S, septum.

Scale bars: $100 \mu \mathrm{m}(\mathrm{B}-\mathrm{I}) ; 50 \mu \mathrm{m}(\mathrm{J}-\mathrm{L})$ 

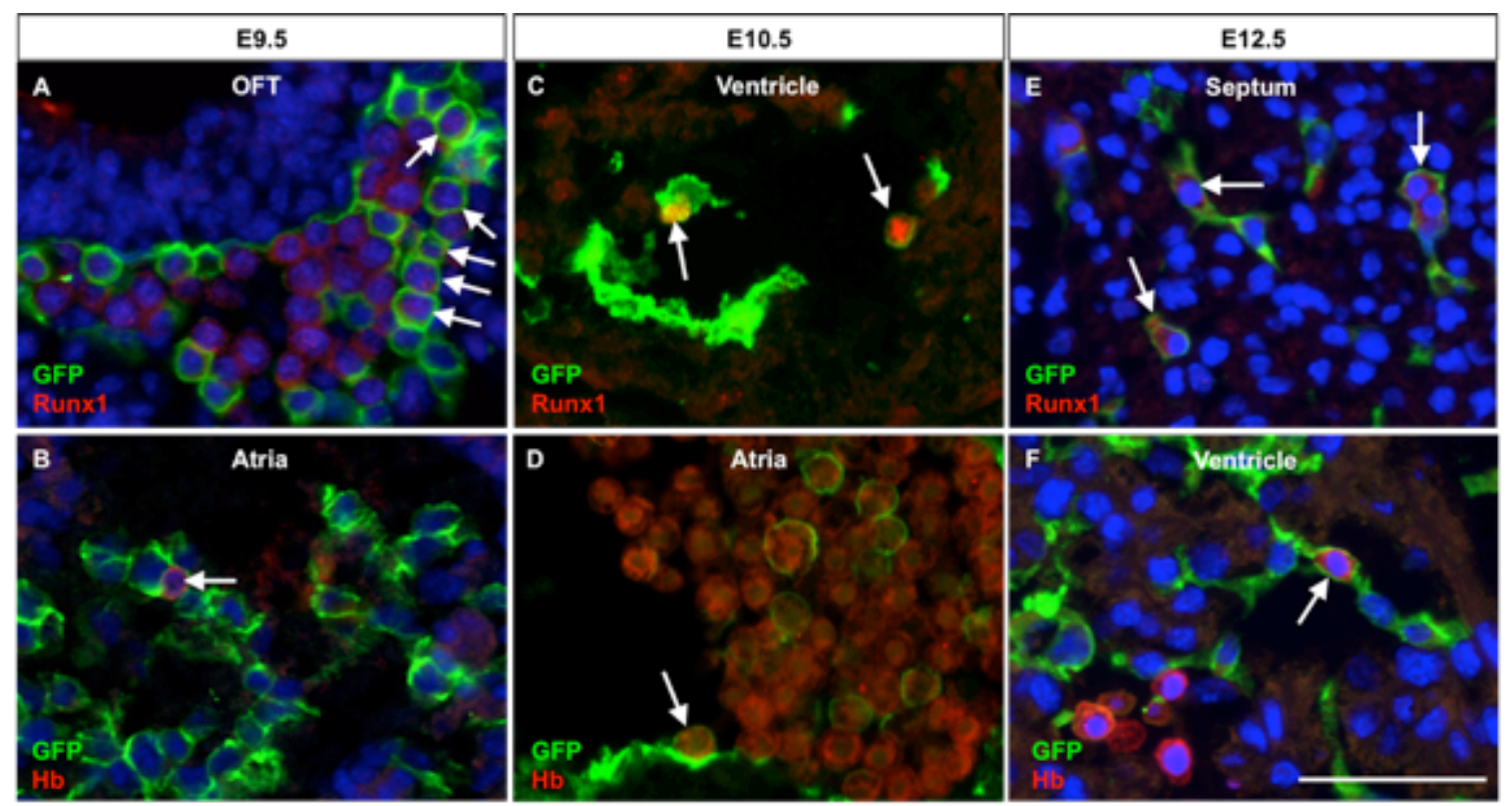

G
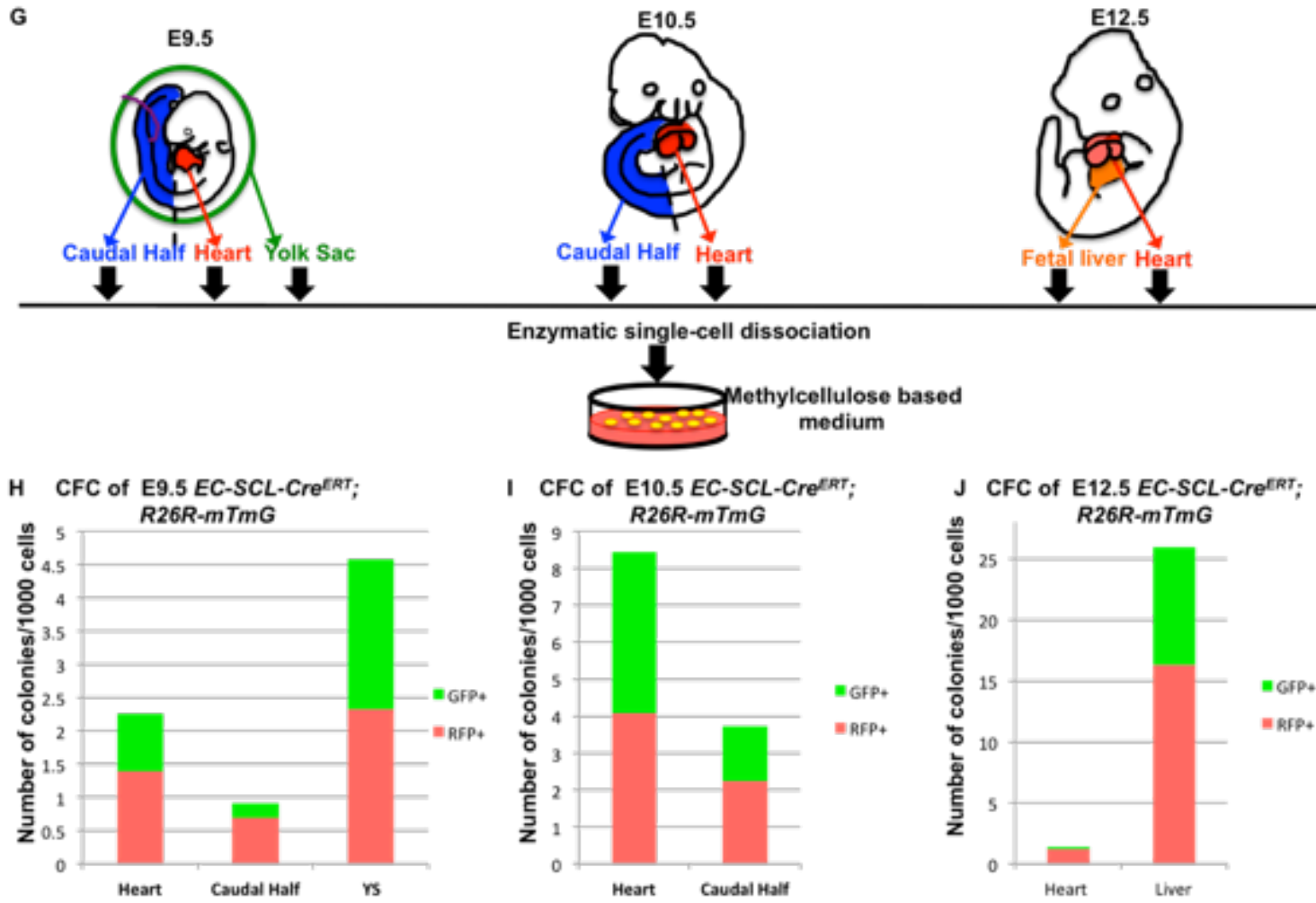

Figure 3.2. $S C L^{E C}+$ precursors contribute to hematopoiesis in the early embryonic heart.

[A-F] IF for GFP and hematopoietic stem cells marker Runx1 and blood cell marker $\mathrm{Hb}$ in sections of hearts at E9.5 (A and B), E10.5 (C and D) and E12.5 (E and F). A,C 
and E show GFP+/Runx1+ cells in the OFT at E9.5 (A, arrows), ventricular endocardium at E10.5 (C, arrows) and in the septal CVECs at E12.5 (E, arrows). B, D and $\mathrm{F}$ show GFP+/Hb+ cells in the atrial endocardium at E9.5 and E10.5 (B and D, arrows) and in ventricular endocardium at E12.5 (F, arrow).

[G] Schematic of colony forming-cells (CFC) assays using cells from whole hearts (red), caudal half (blue), yolk sac (green) and fetal liver (orange) of E9.5, E10.5 and E12.5 embryos.

[H-J] Bar graph shows the number of GFP+ (green) and RFP+ (red) CFU-Mix colonies that grew from the cells of hearts and other hematopoietic organs from E9.5 $(\mathrm{H}$,

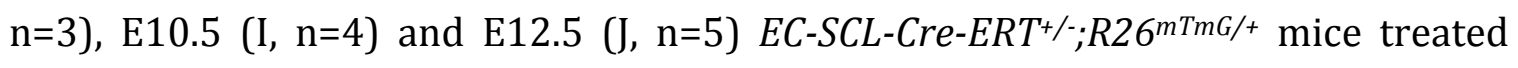
with tamoxifen at E7.5. YS, yolk sac.

Scale bar: $50 \mu \mathrm{m}(\mathrm{A}-\mathrm{F})$ 

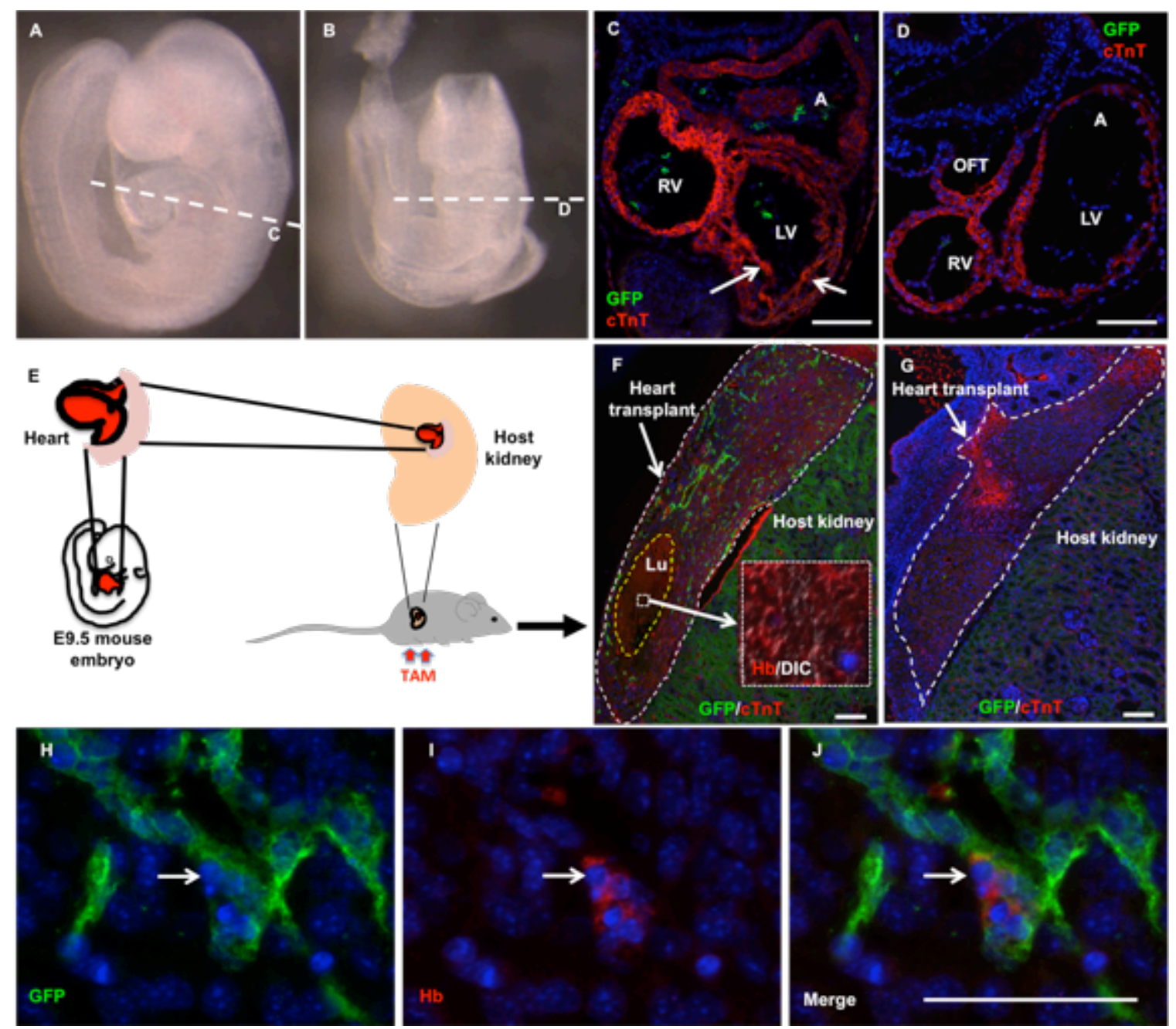

Figure 3.3. Ablation of $S C L^{E C}+$ precursors impairs hemo-vasculogenesis and heart development.

[A-B] Low-dose of tamoxifen injected at E7.5 led to delayed growth of the DTA+ $(E C-$ SCL-Cre-ERT $T^{+/-;} R 26^{\left.D T A /-; R 26^{m T m G /-}\right)}$ mice at E9.5(B), whereas control embryos (ECSCL-Cre-ERT+/;R26 $6^{m T m G /-}$ ) did not display any abnormality (A). Dashed lines indicate the position of the heart sections shown in C and D.

[C-D] IF for GFP (green) and cTnT (red) show that control mice developed a normal heart with a subset of the endocardium labeled by GFP expression. (C) GFP+ cells were absent in DTA+ mice. 
[E] Schematic of embryonic heart transplantation. The whole heart from E9.5 mouse embryos was dissected and transplanted under the kidney capsule of an adult wild type host mouse. After surgery, the host mouse was injected with tamoxifen to induce cre expression with subsequent DTA and reporter expression. The transplanted hearts and host kidneys were harvested 7 days after surgery.

[F-G] IF for GFP (green) and cTnT (red) show that the control transplanted heart developed GFP+ endocardium ( $F$, within the white dashed line). The yellow dashed line indicate a lumen (Lu), filled with $\mathrm{Hb}+$ blood cells (in red, shown in the inset). The DTA+ transplanted heart completely lost the lumen structure and GFP+ cells. (G, white dashed line)

[H-J] IF for GFP and Hb show a GFP+/Hb+ blood island in the section of the control transplanted heart. (arrows)

Scale bars: $100 \mu \mathrm{m}(\mathrm{C}-\mathrm{D}, \mathrm{F}-\mathrm{G}): 50 \mu \mathrm{m}(\mathrm{H}-\mathrm{J})$ 

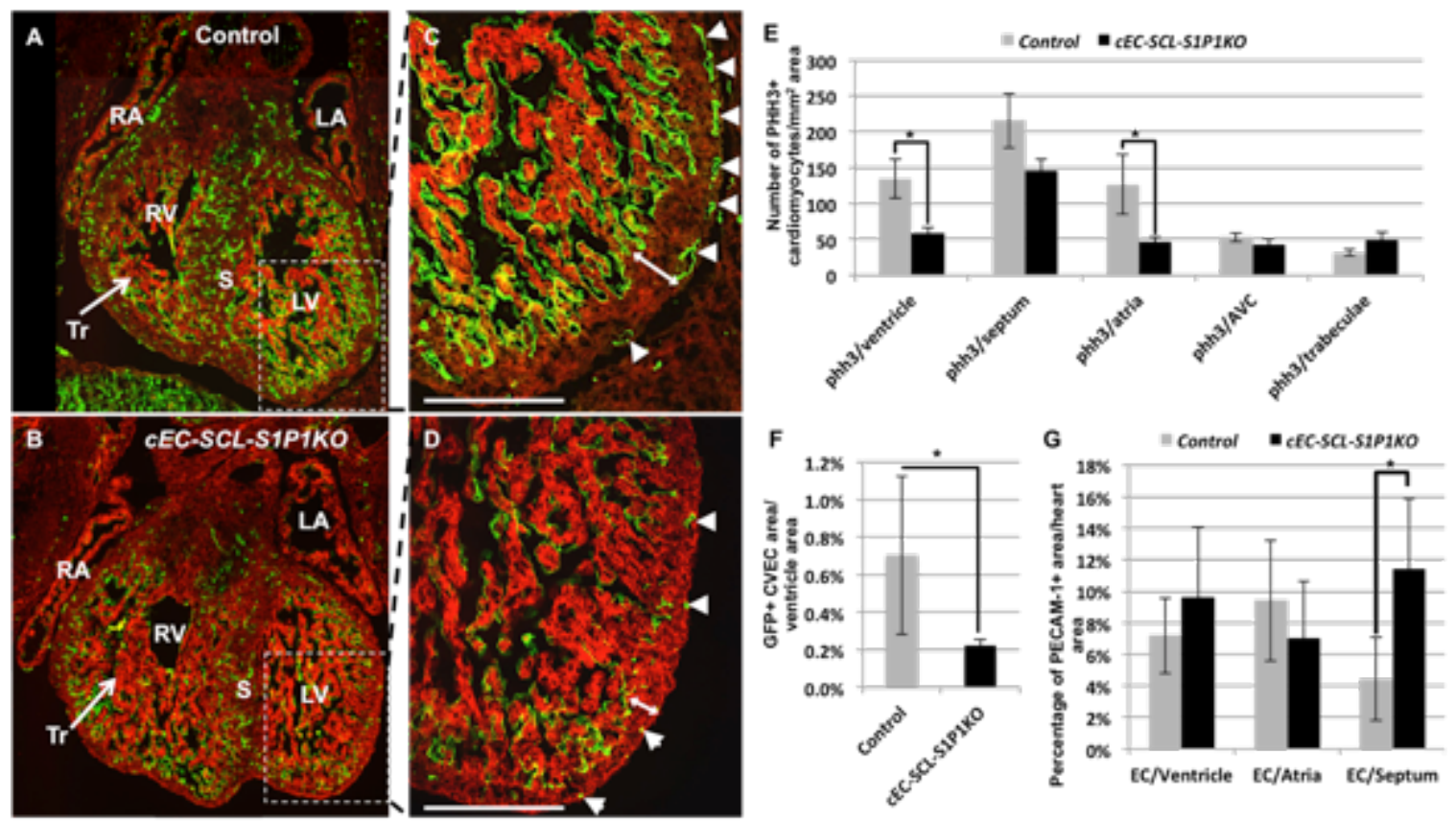

Figure 3.4. $S 1 P 1$ in $S C L^{E C}+$ precursors is crucial for normal heart development.

[A-D] A and B show fluorescent images of heart sections from control (EC-SCL-Cre-

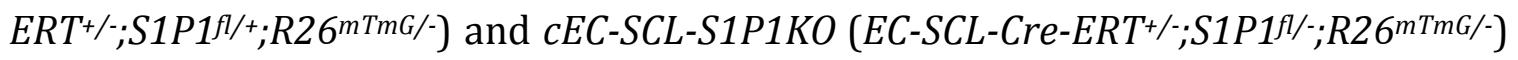
mice. The $S C L^{E C+}$ cells were labeled by GFP expression. Otherwise, the cells express RFP. C and D show the white dashed squares at higher magnification. Comparing to the heart from control mice, the heart of $c E C-S C L-S 1 P 1 K O$ mice (B and D) show thickened trabeculae (A and B, arrows), thin ventricular walls ( $C$ and $D$, double head arrows) in both ventricles, and fewer ventricular coronary vessels (triangles).

[E] Quantification of the number of proliferating phosphorylated histone H3 (phh3) + cTnT+ cells in different cardiac structures of E12.5 control $(\mathrm{n}=6)$ and $c E C-S C L-$ S1P1KO $(\mathrm{n}=5)$ mice. The $C E C$-SCL-S1P1KO hearts showed significantly reduced 
proliferation in the myocardium of ventricles and atria, but not in the septum, AVC or trabeculae. ${ }^{*} \mathrm{p}<0.05$

[F] Measurement of the area of GFP+ ventricular CVECs/ ventricle area in hearts sections of E12.5 control $(\mathrm{n}=5)$ and $c E C$-SCL-S1P1KO $(\mathrm{n}=5)$ mice showed less CVECs in the $c E C$-SCL-S1P1KO heart than the control heart. * $\mathrm{p}<0.05$

[G] Measurement of the area of PECAM-1+ cells in different structures of hearts from E12.5 control $(\mathrm{n}=4)$ and $c E C-S C L-S 1 P 1 K O(\mathrm{n}=4)$ mice show increased septal CVECs in the $c E C$-SCL-S1P1KO heart. There were no differences in the endocardium of ventricles and atria. ${ }^{*} \mathrm{p}<0.05$

Scale bars: $200 \mu \mathrm{m}(\mathrm{A}-\mathrm{D})$ 


\section{Chapter 4. Discussion}

\section{Embryonic origin of renal ECs}

Whether renal endothelium originates intrinsically within the embryonic kidney or from extrarenal precursors has been controversial for decades. The inter-species embryonic kidney grafts have been developed to study the origin of the kidney vasculature. ${ }^{150}$ The variable contribution from host or donor to the vasculature of grafted kidneys raised the argument that this system created a very artificial environment. ${ }^{109}$ When embryonic kidneys from rodent or chick were transplanted into the quail chorioallantoic membrane ${ }^{150}$ or under kidney capsule of newborn mice 15 where vascularization is actively occurring, endothelial cells derived from the host were identified within the vasculature of the grafted embryonic kidneys. However, when embryonic kidneys were transplanted into the anterior eye chamber ${ }^{105}$ or under the kidney capsule of adult rodents ${ }^{15}$ the graft developed its own vasculature without cells from the host. In our lineage tracing studies, SCL+ precursors labeled at E6.5, before the kidney starts to form, contribute to the kidney vasculature. Although, we cannot exclude the contribution of extra-renal precursors to the kidney vasculature, it is possible that these early precursors migrated to the intermediate mesoderm, before the metanephric mesenchyme condenses. In our transplantation studies, the $S C L+$ precursors within E12.5 mouse kidney are able to develop vascular endothelium de novo. When they were genetically ablated from the graft, the vascular endothelium was significantly reduced, while cells from the adult host kidney did not compensate the loss of endothelium. These experiments demonstrated that the metanephric mesenchyme of the early embryonic kidney 
possesses sufficient endothelial precursors to develop the vasculature intrinsically. As mentioned in Chapter 2, the embryonic kidneys in the culture condition cannot develop normal vasculature. By identifying the vascular precursors, we will be able to study the environmental factors and signaling molecules guiding their development and assembly. Further, this information could be ultimately applied to build up a kidney with functional vasculature using cells from the patients who need kidney transplants.

\section{Hemo-vasculogenesis in early embryonic kidneys}

In Chapter 2, we identified hemo-vasculogenesis in E12.5 to E13.5 mouse kidneys, demonstrating that the mammalian embryonic kidneys are able to generate their own vessels and blood, before the kidney vasculature connects to the general circulation. In this section, the following questions will be discussed: 1) How is hemo-vasculogenesis initiated, 2) what is the function of hemo-vasculogenesis, and 3) why is hemo-vasculogenesis transient.

\subsection{Initiation of hemo-vasculogenesis in the developing kidney}

In vitro ES cell culture data suggested that HIF1 induced by hypoxia promoted differentiation of hemangioblasts and accelerated vascular development. ${ }^{151}$ In the kidney, the hypoxic condition resulted from lack of blood flow could be one of the first cues to initiate hemo-vasculogenesis. The mesodermal stem cells within the metanephric mesenchyme may differentiate to hemo-vascular precursors in response to $\mathrm{O}_{2}$ deprivation and form the first vascular endothelium plexus before 
blood perfusion. (Figure 4.1) This hypothesis needs to be verified in both in vitro and in vivo systems.

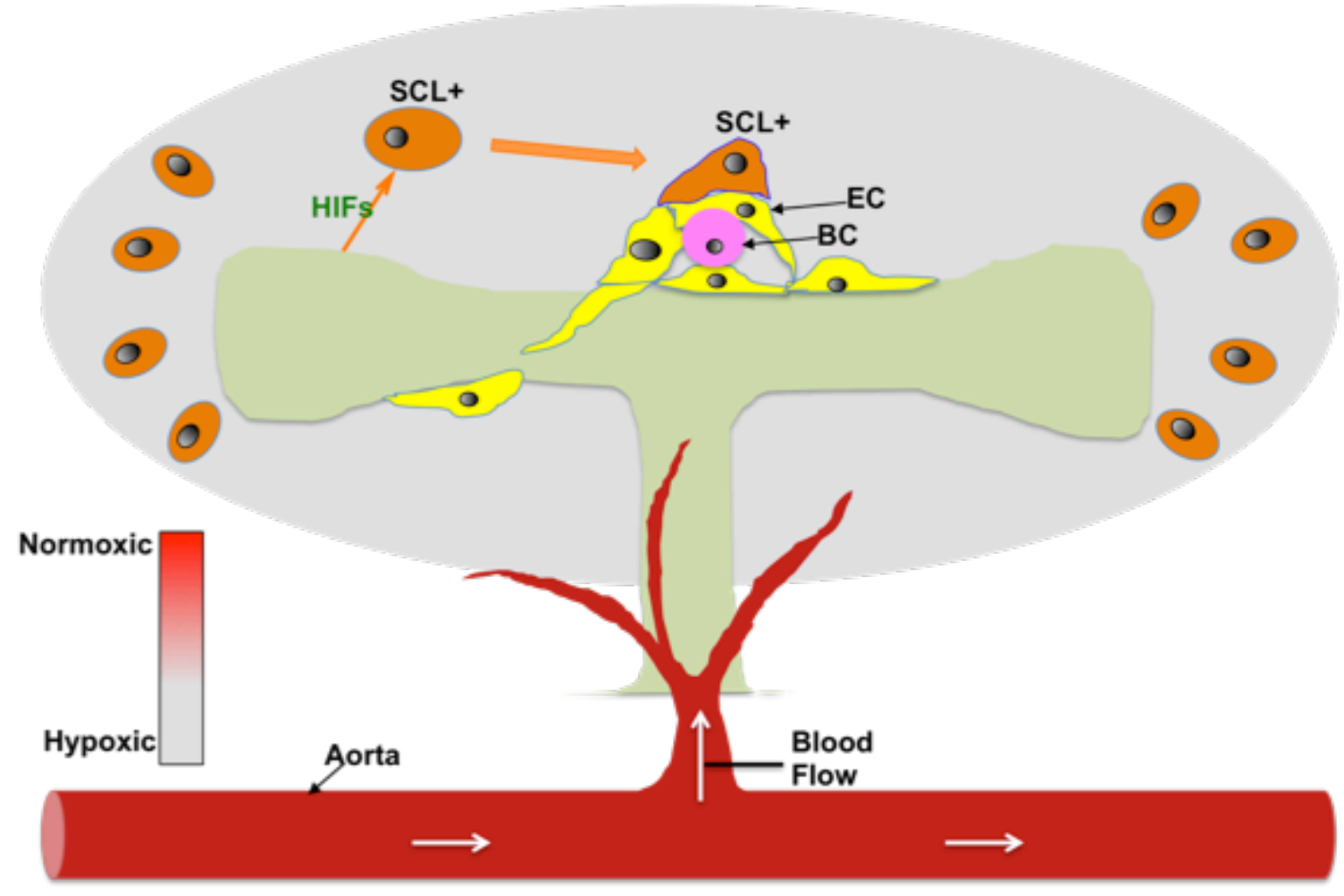

Figure 4.1. Schematic diagram of the initiation of hemo-vasculogenesis in the

E11.5-E12.5 embryonic kidney. Before a connection between the kidney and the blood circulation from aorta is established, the $S C L+$ precursors in the metanephric mesenchyme differentiate to endothelial cells (EC) and blood cells (BC), likely in response to HIFs released by ureteric bud (UB). ${ }^{32}$

\subsection{Possible function of hemo-vasculogenesis}

In addition to oxygen, blood flow also provides growth factors and mechanical forces to vessels and tissues, which are critical for the architecture of the vasculature. However, before establishment of an effective blood circulation, the 
nascent kidney vascular plexus needs to undergo remodeling to form functional vessels, including EC proliferation, regression and mural cell coating, which raises the question: how do immature vessels in the early kidney "self-start" to form functional structures. As the vascular ECs expand, the developing epithelium may produce growth factors to guide the development of new vessels accommodating to ureteric bud branching and nephrogenesis, such as VEGF and HIFs. ${ }^{32}$ Furthermore, it is possible that the blood cells generated by hemo-vasculogenesis function as a regulator for vascular development. For instance, erythrocytes (or red blood cells) not solely function in gas exchange. The embryonic erythrocytes release S1P to the plasma and target endothelial S1P1 to regulate vascular development. ${ }^{58}$ Moreover, the macrophages can release angiogenic factors to guide connection between two sprouting vessels. ${ }^{152}$ Thus, we propose that hemo-vasculogenesis may function as a "self-guided" mechanism during the early vascular development of the kidney, before the establishment of an effective blood circulation. In this model, the SCL+ precursors differentiate to ECs, which form the nascent vascular tubes, and blood cells within the lumens. While the kidney vascular plexus expand through angiogenesis, these blood cells may generate signaling molecules to guide the proper vascular formation and maintain the structures by interacting with the chemotactic signaling molecules from the growing epithelium.

As shown in Figure 2.1 (Chapter 2), the kidney starts to form nascent endothelial tubes with blood cells in the lumens at E13.5. At this stage, the primary vascular plexus with blood cells established by hemo-vasculogenesis may undergo selfguided angiogenesis to expand the vascular network within the rapidly growing 
kidney. (Figure 4.2) Additionally, we found cells co-expressing SCL, stem cell marker and hematopoietic markers in the metanephric mesenchyme of E13.5 kidneys (Figure 2.2), suggesting an ongoing hemo-vasculogenesis in this region. (Figure 4.2) At this time point, some of the kidney vessels may start to fuse to the systemic vasculature. However, it is not necessarily equal to the establishment of blood circulation within the kidney vasculature. Therefore, the kidney vascular development still needs signaling from the blood cells derived from the SCL+ precursors to guide their development.

Although we did not find hematopoietic precursor cells derived from the SCL+ precursors in vivo from E14.5 to E15.5, the hemo-vasculogenesis in the kidney at this stage cannot be excluded based on data in this these. It is possible that the blood flow from the general circulation started to enter the kidney vasculature, which "flushed" the $S C L+$ lineage blood cells (if any) out of the kidney vasculature. Meanwhile, we identified smooth muscle cells and renin cells coating the renal arteries by immunohistochemistry at this stage, indicating that the kidney vessels were undergoing maturation. The growth factors and the hemodynamic forces from the blood flow may further finely tune the differentiation and assembly of the mural cells. However, the hemo-vasculogenesis in the kidney may eventually cease due to the higher concentration of oxygen, regulatory molecules and the hemodynamic forces coming from the blood flow. 


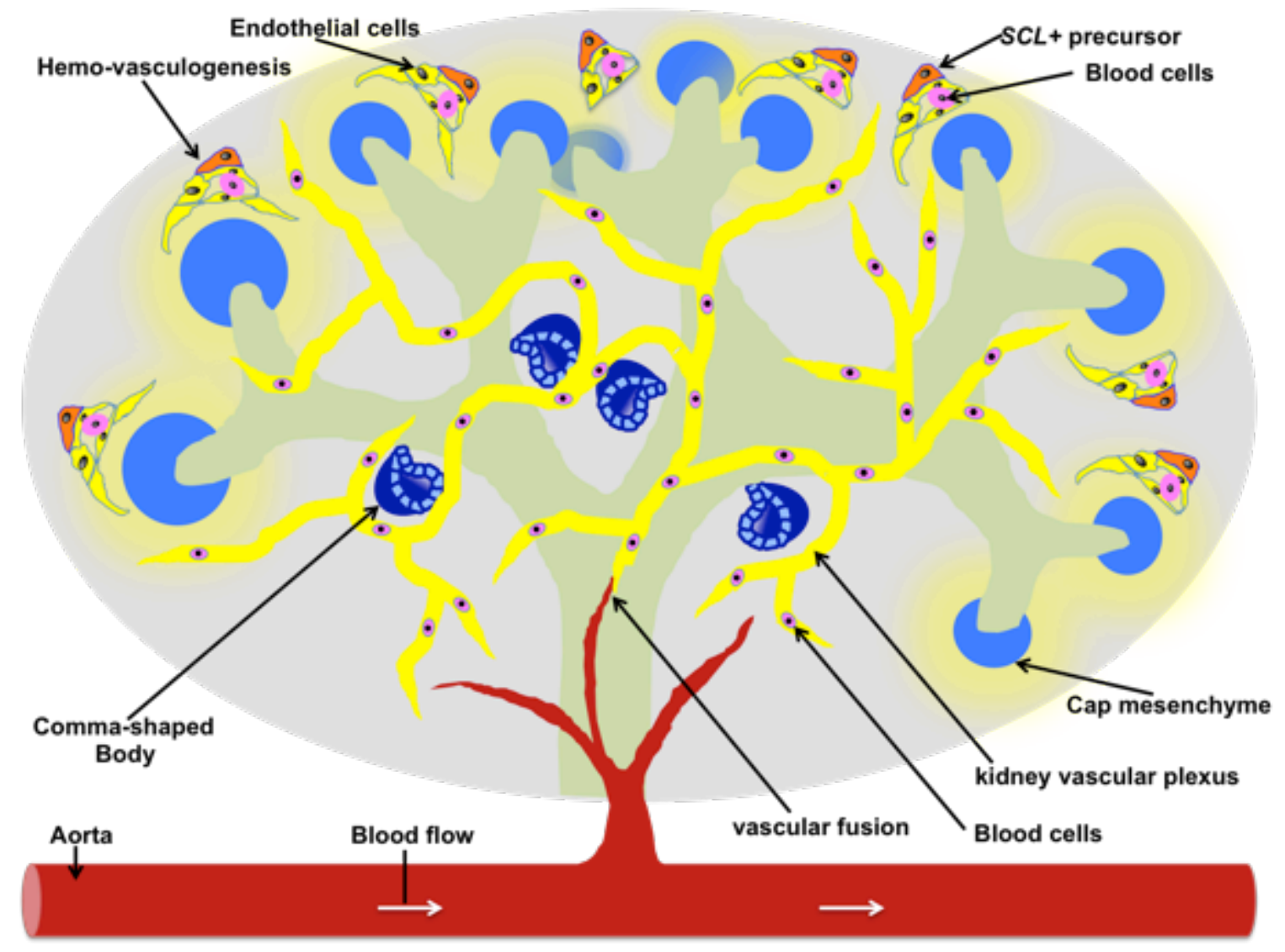

Figure 4.2. Schematic diagram of kidney vascular development from E12.5 to

E13.5. From E12.5 to E13.5, the primary kidney vascular plexus (in yellow) with blood cells (in pink) within the vessels formed by hemo-vasculogenesis undergo angiogenesis. Some vessels may start to fuse with the systemic vessels (in red). In addition, hemo-vasculogenesis occurs in the cortex region. $S C L+$ precursors (in orange) differentiate to endothelial cells (in yellow) and blood cells (in pink). The cap mesenchyme and developing nephrons are labeled in blue, and the ureteric brunches are labeled in pale green.

To summarize our proposed model, the temporal hemo-vasculogenesis in the early embryonic kidney may serve as a "self-guided" machinery during the initiation of 
vascular development. It may be triggered by the hypoxic condition in the early embryonic kidneys to form a vascular plexus with blood cells. Before the establishment of blood circulation, these blood cells provide signaling molecules within the vessel lumens to guide the development of kidney vascular plexus. When the blood flow from the general circulation enters the kidney vasculature, the hemovasculogenesis gradually ceases probably due to the incoming oxygen, growth factors and the hemodynamic forces from the blood flow.

\section{Hemo-vasculogenesis in the embryonic heart}

Similar to the kidney, we found in the heart that the $S C L+$ precursors contribute to hemo-vasculogenesis in the endocardium and the immature coronary vascular plexus in the early embryonic hearts. However, the mechanism underling this process remains largely unknown.

From our CFC data (Figure 3.2), we found the most abundant early hematopoietic colonies derived from the heart at E9.5 and E10.5. Coincidently, the myocardium expresses high level of HIF1 $\alpha$ from E8.5 to E10.5, in response to hypoxic condition. 153 Similar to our hypothesis in the early embryonic kidney, the hypoxic condition could be one of the first cues to trigger the hematopoiesis by the $S C L+$ precursors. However, the function of the blood cells generated during this process is unknown. Unlike vascular EC, the endocardium possesses different morphology and gene expression. Whether its development requires hemo-vasculogenesis is unclear. To address this question, a genetic approach can be used to specifically delete Runx1, the master regulator of hematopoiesis, ${ }^{143}$ from the early endocardium. By crossing 
our inducible $E C$-SCL-Cre-ERT+/- mouse to a Runx $1^{f / f l}$ mouse, we can study the endocardium development with/without hematopoiesis in vivo and in our crosstransplantation system. If the development of endocardium from was affected by the deletion of Runx1 from E9.5 to E10.5, when the hematopoiesis in the heart reaches a peak, it suggests that the blood cells generated by the $S C L+$ precursors regulate the endocardial development. If not, the hematopoiesis may not function to regulate the endocardium. However, it is also possible that the hematopoiesis regulates the development of myocardium, which can be validated in the same experiment.

Secondly, the coronary vascular development starts at E10.5, which may also be stimulated by the hypoxic condition in the heart. Similar to the kidney vasculature, the coronary vasculature first form vascular plexus, which need to undergo remodeling to form a functional vascular structure. ${ }^{154,155}$ From E11.5 to E13.5, the coronary plexus forms and expands rapidly to accommodate with the dramatic cardiac growth resulted from proliferation of cardiomyocytes. During this period, there is no blood flow in the coronary vasculature. ${ }^{155}$ Although hematopoietic precursors were identified in the coronary plexus in the subepicardial region and within the ventricular septum, ${ }^{42,78}$ the origin and the function of these cells are unknown. In chapter 3, our data showed that the $S C L+$ precursor gave rise to both hematopoietic precursor cells and the coronary vessels in the E12.5 and E13.5 hearts. (Figure 3.1 and 3.2) Similar to the kidney vasculature, it is possible that the hemo-vasculogenesis in the coronary vascular system function as a "self-guided" 
machinery to regulate its own development before connection to the general circulation.

\section{Role of S1P1 signaling in kidney vascular development}

In Chapter 2, we demonstrated that the S1P1 receptor functions autonomously in ECs to regulate critical steps during kidney vascular development, including EC proliferation, mural cell coating, glomerular capillary formation and lymphatic vessel formation. These processes are finely regulated by interactions between multiple signaling pathways, which remain largely unknown.

\subsection{Possible role of S1P1 in the kidney vascular development}

Earlier in vitro and in vivo studies suggested that endothelial S1P1 function as a vascular stabilizer through inhibiting angiogenic sprouting, promoting cell-cell

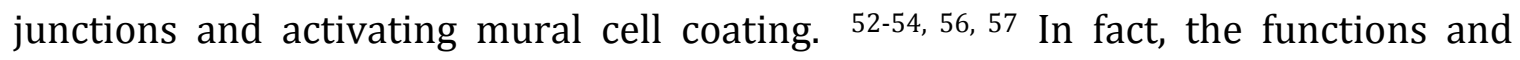
expression level of S1P1 differs upon the dose of ligand, flow mediated forces and localization of EC in different type of vessels and organs. (Blaho, Hla 2014) Whether S1P1 is expressed in the SCL+ precursors or the earliest ECs during hemovasculogenesis and whether it regulates the nascent vascular formation is unclear. Therefore, a detailed expression profile of S1P1 revealed by in situ hybridization in each segment of kidney vessels at different developmental stages is necessary for understanding its role in the kidney vascular development.

Microarray data of E15.5 mouse kidneys showed that S1P1 is highly expressed in the ECs when coupled with other cell types of the embryonic kidney. ${ }^{156,157}$ Our study further demonstrated that the endothelial S1P1 inhibited EC proliferation in 
the embryonic kidneys, which explained the EC hyperplasia in the kidneys of $i E C$ SCL-S1P1KO mice. Whether S1P1 directly regulates EC proliferation through $\mathrm{G}_{\mathrm{i}}-\mathrm{Rho}$ GTPase signaling or synergistically with other signaling pathways, such as VEGF signaling, need to be studied. Further, mural cell coating, a process that involves differentiation, proliferation, migration and stabilization of mural cells is regulated by Angiopoietin-1/Tie2, PDGF/PDGFR, Eph/Ephrin, TGF- $\beta$ and S1P/S1P1 signaling pathways. Yet, our knowledge on the interactions among these signaling pathways is incomplete. In the kidneys of $i E C-S C L-S 1 P 1 K O$ mice, the SMCs of renal arterioles showed abnormal arrangement. Whether their differentiation and proliferation were affected by the knockout of EC S1P1 is unknown. Previous studies showed that the endothelial S1P1/Gi/Rac signaling pathway activated trafficking of N-cadherin to form cell-cell adhesion between ECs and mural cells. ${ }^{158}$ Thus the disarrangement of the SMC layer of the kidney arterioles in $i E C-S 1 P 1 K O$ mice may be due to perturbation of $\mathrm{N}$-cadherin junctions. Those studies are needed in the kidney to gain a better understanding of renal vascular development or its disarrangement during disease. Understanding the signaling pathways will likely allow the design of therapeutic agents to prevent and/or treat patients suffering from chronic kidney disease and/or hypertension.

Glomerular capillaries are unique vascular structures in the kidney, which form a complex capillary network within each glomerulus. A vasculogenesis model for initiation of glomerular capillary formation has been suggested. ${ }^{109}$ In this model, the individual endothelial progenitor cells are believed to stream into the vascular clefts of the S-shaped glomeruli in response to chemotactic VEGF-A secreted by the 
developing podocytes. After the influx of EC progenitors, these cells proliferate and rearrange to form the first capillary lumen. ${ }^{109}$ Different from this model, in Sshaped glomeruli, we observed filopodia-like structures in leading ECs and red blood cells in the lumens formed by the invading ECs, suggesting that at least a part of the invading ECs already formed vessel lumens with blood cells inside. (Figure 4.3) Whether hemo-vasculogenesis or angiogenic sprouting is involved in this process is unknown. To test this, three-dimensional reconstruction of the developing glomerular capillaries will be needed.

After the initial capillary lumen formation, intussusceptive angiogenesis is a major contributor to the glomerular capillary growth, which was observed in both developing glomeruli and injured glomeruli undergoing capillary repairing. ${ }^{159-161}$ In this process, two adjacent ECs protrude to form a trans-capillary pillar structure into the vessel lumen. Then projections from mesengial cells and interstitial fibers invade into this structure, resulting in splitting of the lumen to new vessels. 109,159 Loss of mesangium or defects in basement membranes that adhere to the mesangium lead to failure of capillary loop formation. The molecular cues guiding formation of trans-capillary pillarsand mensangium protrusion are unknown. In our study, we discovered capillary shunts with abnormal mesangium in the kidneys of iEC-SCL-S1P1KO mice, suggesting a potentially crucial role of endothelial S1P1 during capillary intussusception. Whether deletion of S1P1 results in absence of pillar structure and/or abnormalities (in migration or protrusion) of the developing mesangium can be examined by scanning electron microscopy. Additionally, knockout of VEGF-A or transcription factors controlling VEGF-A expression from 
podocytes lead to capillary defects resembling those found in our studies. ${ }^{162}$ Thus, the interplay between S1P1 and VEGF signaling may guide the formation of glomerular capillary loops.
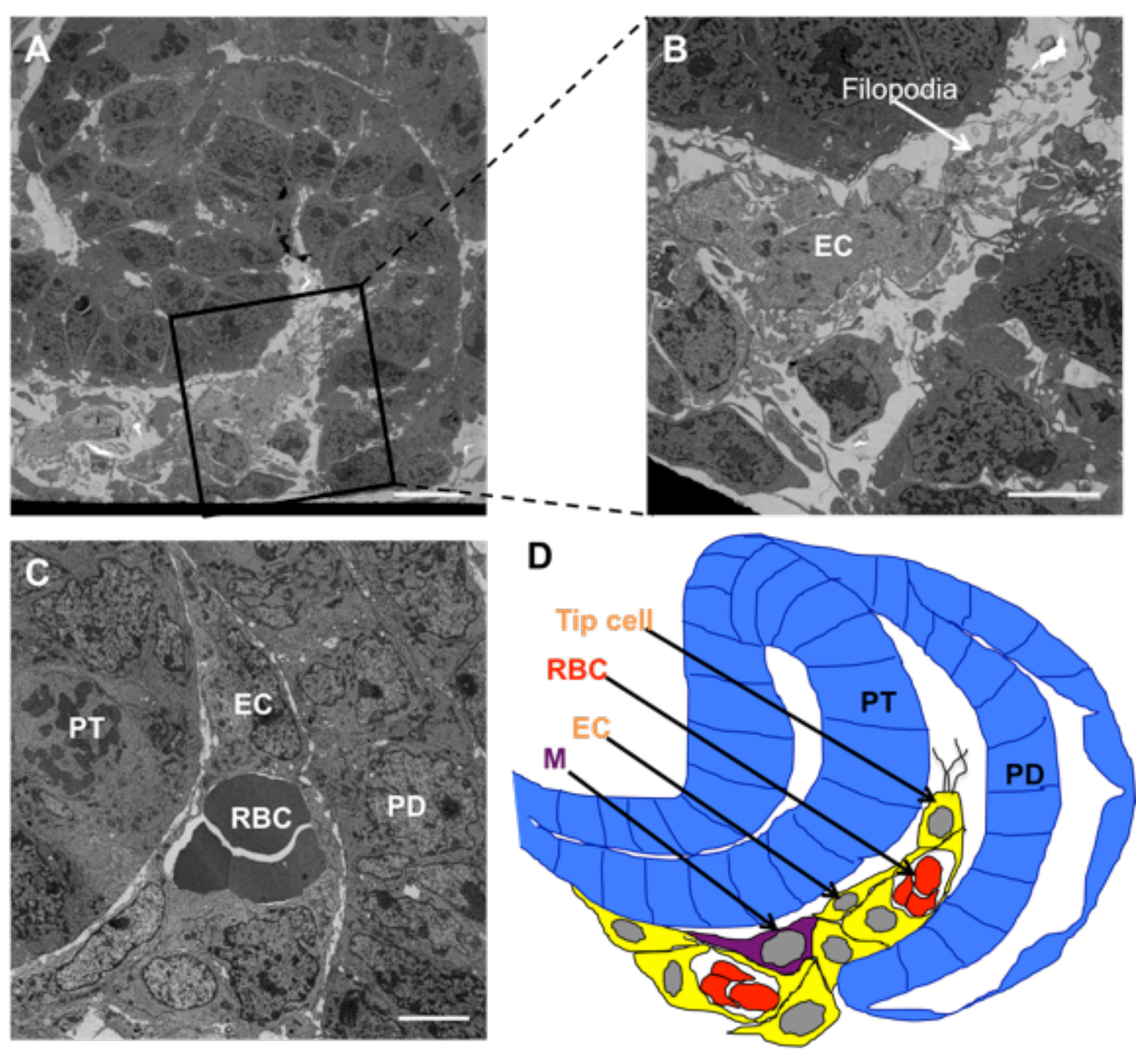

Figure 4.3. Development of glomerular capillaries in S-shaped glomerulus. [A-

C] $50 \mathrm{~nm}$ embryonic mouse kidney sections under transmission electron microscopy showing "tip cell" like endothelial cell (EC) (A, squared) with filopodia (B) and red blood cells (RBCs) in the vessel lumen formed by the invading ECs (C) in S-shaped glomeruli. [D] Schematic graph demonstrating that ECs (in yellow) form 
tip cell and vessel lumens with RBCs (in red) in the vascular cleft of S-shaped glomerulus (in blue). M, mesengial cell. PD, podocyte. PT, proximal tubule. Scale bar: A-C, $5 \mu$ m. (Unpublished data, by Yan $\mathrm{Hu}$ )

\subsection{Possible role of S1P1 in renal lymphatic development}

An interesting finding from our study is the lack of lymphatic vessels in the kidneys of $i E C-S C L-S 1 P 1 K O$ mice. Albeit our lineage tracing study clearly demonstrated that $S C L+$ precursors did not contribute to the renal lymphatic vessels. Therefore, S1P1 in vascular ECs may function in a non-cell-autonomous manner to regulate the lymphatic development. Both vascular systems are anatomically closely associated during development and adulthood. In addition, lymphatic formation follows the growth pattern of blood vessels. It is known that angiogenesis and lymphatic development share many common regulatory signaling pathways, including S1P signaling, VEGFR3/VEGF-C, Notch signaling, angiopoietin(Ang)-2-Tie2 and flowmediated signaling. ${ }^{57,163-165}$ It is possible that vascular endothelial S1P1 is involved in regulation of ligand secretion by vascular ECs, which guides lymphatic development. Here we will discuss several ligands that may be altered by the deletion of endothelial S1P1.

First of all, it is possible that the deletion of S1P1 reduced the expression and exocytosis of Ang-2 by endothelial cells, which in turn impaired the lymphatic development. The Ang-2-Tie2 signaling pathway has been shown to promote lymphangiogenesis. ${ }^{165}$ And evidence from the cultured human ECs and LECs showed that S1P stimulate secretion of angiopoietin-2 through activating S1P1. 166 
Therefore, the paracrine factor Ang2 seems to connect the endothelial S1P1 to the development of lymphatics. To validate this model, the expression level of Ang-2 in the kidneys of $i E C-S 1 P 1 K O$ and control mice can be tested by RT-PCR, in situ hybridization and immunostaining. The exocytosis of Ang-2 can be tested by ELISA.

Secondly, the endothelial S1P1 may regulate lymphangiogenic factors through HIF$1 \alpha$. In blood vessels, deletion of S1P1 up-regulates the level of HIF- $1 \alpha$, which induces VEGF-A expression and leads to endothelial hyperplasia. 167 During lymphangiogenesis, HIF- $1 \alpha$ regulates VEGF-C expression in the LECs. ${ }^{168}$ Whether the absence of lymphatics in the kidney of $i E C-S 1 P 1 K O$ mice is associated with an elevated HIF-1 $\alpha$ needs to be investigated. Further, the expression level of VEGF-C needs to be tested in the $I E C-S 1 P 1 K O$ and control mice to validate this hypothesis.

Finally, the S1P1 deletion in vascular ECs may alter the level of Dll4 ligand secretion and lead to the abnormal lymphatic development. The Dll4/Notch1 signaling pathway has been shown to play a central role in the LEC differentiation, migration, lymphatic patterning and crosstalk between blood vessels and lymphatic vessels. ${ }^{163,}$ 169,170 Although there is no experimental evidence supporting that the endothelial S1P1 regulate Dll4 secretion in the kidney. Previous studies performed in endometrial of pregnant sheep showed that S1P controlled Dll4 expression in the vasculature. ${ }^{171}$ Since the expression pattern of Dll4 in the endometrial vasculature overlaps with that of S1P1, Dll4 secretion may be controlled by S1P-S1P1 signaling. 171 The Dll4 expression can be tested in the kidneys of our $i E C$-S1P1KO and control mice by RT-PCR, in situ hybridization and immunostainings to investigate whether it is altered by the deletion of S1P1. 
To summarize the above hypothesis, the endothelial S1P1 in the kidney may function as a regulator of lymphatic vascular development through paracrine factors secreted by the ECs.

\section{Role of S1P1 in heart development}

Collective data from studies in the avian and mouse models showed that the myocardium and coronary vascular development are finely regulated by interactions among the signaling pathways among each structure, including endocardium, myocardium, coronary vacsulature and epicardium. ${ }^{76,148}$ To study the role of S1P1, which is widely expressed in multiple cardiac cells types, the functions of S1P1 in each cell type need to be analyzed. In Chapter 3, we showed that S1P1 expressed by cardiac endothelium derived from the $S C L+$ precursors in the heart controls the development of coronary vasculature and myocardium. Timed deletion of S1P1 from early $S C L+$ precursors led to abnormal ventricular myocardium and impaired ventricular coronary vessels. The detailed mechanisms underling the regulation of coronary vascular and myocardial development by endothelial S1P1 is currently lacking. Here we will discuss several possible roles of endothelial S1P1 in the development of endocardium, coronary vasculature and myocardium.

\subsection{Possible role of S1P1 in coronary vascular development}

In our study, we observed significant reduction of coronary vessels within the ventricular wall in response to the deletion of endothelial S1P1. It is has been reported that the endocardium give rise to a sub set of coronary vessels through angiogenesis, which is regulated by VEGF signaling pathway between the 
myocardium and endocardium. ${ }^{131}$ And S1P1 is known to be an angiogenic inhibitor through regulating the interaction between VE-Cadherin and VEGFR2. ${ }^{56}$ Therefore, the reduction of coronary vessels could be resulted from impaired angiogenesis from the endocardium. To validate this hypothesis, the expression level and function of VE-Cadherin and VEGFR2 can be tested in the endocardium of both control and the knockout animals in vivo or in vitro.

Additionally, the coronary vessels originating from the reprogrammed sinus venous (SV) endothelium were affected by the deletion of endogenous S1P1. This process involves dedifferentiation of the venous ECs, (hemo-)vasculogensis and angiogenesis, which are finely regulated by paracrine factors secreted by both myocardium and epicardium. 139 Since we identified abnormalities in the ventricular myocardium, it is unclear whether the coronary defects are caused by intrinsic regulation by S1P1 or secondary to the myocardium defects, or both. To understand the role of S1P1 during these processes, a detailed expression profile of S1P1 in the SV ECs and coronary vascular plexus by in situ hybridization and immunohistochemistry is needed at first. Further, timed deletion of endothelial S1P1 from SV or endocardial lineage using inducible Cre-loxp mouse system would help us to better understand the role of S1P1.

In our $C E C$-SCL-S1P1KO mice, the abnormalities in the heart start to show after the development of the coronary vasculature. From E9.5 to E13.5, the endocardium of the knockout mice showed no alterations. It is possible that the endothelial S1P1 function at later stages to regulate the endocardium. To confirm this, using a mouse expressing Cre recombinase driven by endocardial specific gene, such as Nfatc1 ${ }^{131}$, 
to cross with S1P1fl/fl mice to delete S1P1 from the endocardium would help us to study the role of endocardial S1P1 during heart development.

\subsection{Possible role of S1P1 in myocardium development}

Our $C E C$-SCL-S1P1KO mice showed a reduced myocardial proliferation, reminiscent of the cardiac defects in the S1P1 null mice, ${ }^{137}$ indicating that the growth of myocardium is regulated by the endothelial S1P1 in a non-autonomous manner. Whether it is controlled by endocardial S1P1, coronary endothelial S1P or both is unclear. Hence, a region specific or timed deletion of endothelial S1P1 will answer this question. Moreover, the downstream signaling cascade controlled by S1P1 needs to the further investigated.

In addition, our $c E C-S C L-S 1 P 1 K O$ mice developed ventricular non-compaction associated with hypertrabeculation. Recently, other groups showed that endothelial Fkbp1a regulate trabeculation and myocardium compaction through activating Notch1 signaling. The mice with conditional knockout of Fkbp1a showed similar cardiac defects to our mice. Whether the S1P1 interact with this signaling pathway is unclear. By isolating the cardiac endothelium and measuring the level of Notch1 using western blot would answer this question.

Given the essential roles of endothelial S1P1 during developmental and pathological processes, it is crucial to understand its downstream signaling in each organ and tissue. Since the drug targeting the S1P signaling pathway, fingolimod (FTY720), was used to treat multiple sclerosis, increasing cases of side effects has been reported, including bradycardia, edema and hemorrhage. By understanding the 
precise regulation by S1P1 in different organs or tissues, we may be able to find a better drug or develop a precise drug delivery strategy to target the disease, leaving the "good" S1P signaling molecules to function properly.

\section{Conclusions and Future Perspectives}

In conclusion, this thesis work has identified the precise cellular origin and mechanisms whereby those early and intermediate hemo-vascular precursors lead to the successful formation of the renal and cardiac vasculature, without which there is no functioning kidney or heart. And the hemo-vasculogenesis in both organs may serve as a self-guided machinery to generate regulatory signaling molecules for vascular development and organogenesis before the vasculature connects to the general circulation. Moreover, the S1P1 expressed in the endothelial cells derived from the hemo-vascular precursors regulates the development of EC, SMCs, lymphatic ECs in the kidney and the development of coronary vasculature and myocardium.

These findings may only depict a piece of puzzles of the development of the vascular network. As pointed in the sections above, there are still a great number of questions to be answered, including the developmental mechanisms underlying the hemo-vasculogenesis and the interactions between S1P1 and other signaling pathways during the vascular development in both organs. By answering these questions, we may be closer to the final goals of repair or de novo regeneration of the kidney and heart, which could benefit children and adult patients suffering from the congenital kidney and heart disease. 


\section{References}

1. Osathanondh, V. \& Potter, E. L. Development of Human Kidney as shown by Microdissection. Ii. Renal Pelvis, Calyces, and Papillae. Arch. Pathol. 76, 277-289 (1963).

2. Osathanondh, V. \& Potter, E. L. Development of Human Kidney as shown by Microdissection. Iii. Formation and Interrelationship of Collecting Tubules and Nephrons. Arch. Pathol. 76, 290-302 (1963).

3. Oliver, J. \& MacDowell, M. in Nephrons and kidneys : a quantitative study of developmental and evolutionary mammalian renal architectonics. $<b r />$ (Hoeber Medical Division, Harper \& Row, New York, 1968).

4. Saxen, L. in Organogenesis of the kidney (Cambridge University Press, 1987).

5. Sequeira-Lopez, M. L. in Kidney Development, Disease, Repair and Regeneration (ed Little, M. H.) 147-162 (Elsevier Science, 2015).

6. Gomez, R. A. \& Sequeira Lopez, M. L. in Assembly of the Vasculature and Its Regulation (ed Tomanek, R. J.) 193-210 (Birkhauser, Boston. Basel. Berlin, 2012).

7. Cullen-McEwen, L., Sutherland, M. R. \& Black, M. J. in Kidney Development, Disease, Repair and Regeneration (ed Little, M. H.) 27-40 (Elsevier Science, 2015).

8. Walker, K. A. \& Bertram, J. F. Kidney Development: Core Curriculum 2011. American Journal of Kidney Diseases 57, 948-958. 
9. Sequeira Lopez, M. L. \& Gomez, R. A. Development of the renal arterioles. J. Am. Soc. Nephrol. 22, 2156-2165 (2011).

10. Mimura, I. \& Nangaku, M. The suffocating kidney: tubulointerstitial hypoxia in end-stage renal disease. Nat. Rev. Nephrol. 6, 667-678 (2010).

11. Tammela, T. \& Alitalo, K. Lymphangiogenesis: Molecular mechanisms and future promise. Cell 140, 460-476 (2010).

12. Holmes, M. J., O'Morchoe, P. J. \& O'Morchoe, C. C. Morphology of the intrarenal lymphatic system. Capsular and hilar communications. Am. J. Anat. 149, 333-351 (1977).

13. Lee, H. W. et al. Expression of lymphatic endothelium-specific hyaluronan receptor LYVE-1 in the developing mouse kidney. Cell Tissue Res. 343, 429-444 (2011).

14. Gattone, V. H.,2nd \& Goldowitz, D. The renal glomerulus and vasculature in 'aggregation' chimeric mice. Nephron 90, 267-272 (2002).

15. Robert, B., St John, P. L., Hyink, D. P. \& Abrahamson, D. R. Evidence that embryonic kidney cells expressing flk-1 are intrinsic, vasculogenic angioblasts. Am. J. Physiol. 271, F744-53 (1996).

16. Sequeira-Lopez, M. L. et al. The earliest metanephric arteriolar progenitors and their role in kidney vascular development. Am. J. Physiol. Regul. Integr. Comp. Physiol. 308, R138-49 (2015). 
17. Lin, E. E., Sequeira-Lopez, M. L. \& Gomez, R. A. RBP-J in FOXD1+ renal stromal progenitors is crucial for the proper development and assembly of the kidney vasculature and glomerular mesangial cells. Am. J. Physiol. Renal Physiol. 306, F249$58(2014)$

18. Sequeira Lopez, M. L. et al. The embryo makes red blood cell progenitors in every tissue simultaneously with blood vessel morphogenesis. Am. J. Physiol. Regul. Integr. Comp. Physiol. 284, R1126-37 (2003).

19. Tanabe, M. et al. Development of lymphatic vasculature and morphological characterization in rat kidney. Clin. Exp. Nephrol. 16, 833-842 (2012).

20. Gordon, E. J., Gale, N. W. \& Harvey, N. L. Expression of the hyaluronan receptor LYVE-1 is not restricted to the lymphatic vasculature; LYVE-1 is also expressed on embryonic blood vessels. Developmental Dynamics 237, 1901-1909 (2008).

21. Sabin, F. R. The lymphatic system in human embryos, with a consideration of the morphology of the system as a whole. Am. J. Anat. 9, 43-91 (1909).

22. Murray, P. D. F. The Development in vitro of the Blood of the Early Chick Embryo. Proceedings of the Royal Society of London.Series B, Containing Papers of a Biological Character 111, 497-521 (1932).

23. Choi, I., Lee, S. \& Hong, Y. K. The new era of the lymphatic system: no longer secondary to the blood vascular system. Cold Spring Harb Perspect. Med. 2, a006445 (2012). 
24. Carmeliet, P. \& Jain, R. K. Molecular mechanisms and clinical applications of angiogenesis. Nature 473, 298-307 (2011).

25. Patel-Hett, S. \& D'Amore, P. A. Signal transduction in vasculogenesis and developmental angiogenesis. Int. J. Dev. Biol. 55, 353-363 (2011).

26. Quaggin, S. E. \& Kreidberg, J. A. Development of the renal glomerulus: good neighbors and good fences. Development 135, 609-620 (2008).

27. Simon, M. et al. Expression of vascular endothelial growth factor and its receptors in human renal ontogenesis and in adult kidney. Am. J. Physiol. 268, F24050 (1995).

28. Woolf, A. S., Gnudi, L. \& Long, D. A. Roles of angiopoietins in kidney development and disease. J. Am. Soc. Nephrol. 20, 239-244 (2009).

29. Floege, J., Eitner, F. \& Alpers, C. E. A New Look at Platelet-Derived Growth Factor in Renal Disease. Journal of the American Society of Nephrology 19, 12-23 (2008).

30. Pardali, E., Goumans, M. J. \& ten Dijke, P. Signaling by members of the TGF-beta family in vascular morphogenesis and disease. Trends Cell Biol. 20, 556-567 (2010).

31. Covello, K. L. \& Simon, M. C. HIFs, Hypoxia, and Vascular Development. Curr. Top. Dev. Biol. 62, 37-54 (2004).

32. Freeburg, P. B. \& Abrahamson, D. R. Hypoxia-inducible factors and kidney vascular development. J. Am. Soc. Nephrol. 14, 2723-2730 (2003). 
33. Lucitti, J. L. et al. Vascular remodeling of the mouse yolk sac requires hemodynamic force. Development 134, 3317-3326 (2007).

34. Garcia, M. D. \& Larina, I. V. Vascular development and hemodynamic force in the mouse yolk sac. Front. Physiol. 5, 308 (2014).

35. Culver, J. C. \& Dickinson, M. E. The effects of hemodynamic force on embryonic development. Microcirculation 17, 164-178 (2010).

36. Sabin, F. R. in Studies on the Origin of Blood-vessels and of Red Blood-corpuscles as Seen in the Living Blastoderm of Chicks During the Second Day of Incubation (Carnegie Institution of Washington, 1920).

37. Xiong, J. W. Molecular and developmental biology of the hemangioblast. Dev. Dyn. 237, 1218-1231 (2008).

38. Isern, J., Fraser, S. T., He, Z. \& Baron, M. H. Developmental niches for embryonic erythroid cells. Blood Cells Mol. Dis. 44, 207-208 (2010).

39. Godin, I. E., Garcia-Porrero, J. A., Coutinho, A., Dieterlen-Lievre, F. \& Marcos, M. A. Para-aortic splanchnopleura from early mouse embryos contains B1a cell progenitors. Nature 364, 67-70 (1993).

40. Li, Z. et al. Mouse embryonic head as a site for hematopoietic stem cell development. Cell. Stem Cell. 11, 663-675 (2012). 
41. Nakano, H. et al. Haemogenic endocardium contributes to transient definitive haematopoiesis. Nat. Commun. 4, 1564 (2013).

42. Jankowska-Steifer, E. et al. Vasculogenic and hematopoietic cellular progenitors are scattered within the prenatal mouse heart. Histochem. Cell Biol. 143, 153-169 (2015).

43. Gering, M., Rodaway, A. R., Gottgens, B., Patient, R. K. \& Green, A. R. The SCL gene specifies haemangioblast development from early mesoderm. EMBO J. 17, 40294045 (1998).

44. Bloor, A. J., Sanchez, M. J., Green, A. R. \& Gottgens, B. The role of the stem cell leukemia (SCL) gene in hematopoietic and endothelial lineage specification. J. Hematother. Stem Cell Res. 11, 195-206 (2002).

45. Porcher, C. et al. The T Cell Leukemia Oncoprotein SCL/tal-1 Is Essential for Development of All Hematopoietic Lineages. Cell 86, 47-57 (1996).

46. Lancrin, C. et al. The haemangioblast generates haematopoietic cells through a haemogenic endothelium stage. Nature 457, 892-895 (2009).

47. Robb, L. et al. Absence of yolk sac hematopoiesis from mice with a targeted disruption of the scl gene. Proceedings of the National Academy of Sciences 92, 70757079 (1995).

48. Shivdasani, R. A., Mayer, E. L. \& Orkin, S. H. Absence of blood formation in mice lacking the T-cell leukaemia oncoprotein tal-1/SCL. Nature 373, 432-434 (1995). 
49. Dekel, B. et al. Kidney, blood, and endothelium: developmental expression of stem cell leukemia during nephrogenesis. Kidney Int. 65, 1162-1169 (2004).

50. Elefanty, A. G., Begley, C. G., Hartley, L., Papaevangeliou, B. \& Robb, L. SCL expression in the mouse embryo detected with a targeted lacZ reporter gene demonstrates its localization to hematopoietic, vascular, and neural tissues. Blood 94, 3754-3763 (1999).

51. Kono, M., Allende, M. L. \& Proia, R. L. Sphingosine-1-phosphate regulation of mammalian development. Biochim. Biophys. Acta 1781, 435-441 (2008).

52. Kono, M. et al. The sphingosine-1-phosphate receptors S1P1, S1P2, and S1P3 function coordinately during embryonic angiogenesis. J. Biol. Chem. 279, 2936729373 (2004).

53. Allende, M. L., Yamashita, T. \& Proia, R. L. G-protein-coupled receptor S1P1 acts within endothelial cells to regulate vascular maturation. Blood 102, 3665-3667 (2003).

54. Liu, Y. et al. Edg-1, the G protein-coupled receptor for sphingosine-1-phosphate, is essential for vascular maturation. J. Clin. Invest. 106, 951-961 (2000).

55. Shoham, A. B. et al. S1P1 inhibits sprouting angiogenesis during vascular development. Development 139, 3859-3869 (2012). 
56. Gaengel, K. et al. The sphingosine-1-phosphate receptor S1PR1 restricts sprouting angiogenesis by regulating the interplay between VE-cadherin and VEGFR2. Dev. Cell. 23, 587-599 (2012).

57. Jung, B. et al. Flow-regulated endothelial S1P receptor-1 signaling sustains vascular development. Dev. Cell. 23, 600-610 (2012).

58. Xiong, Y., Yang, P., Proia, R. L. \& Hla, T. Erythrocyte-derived sphingosine 1phosphate is essential for vascular development. J. Clin. Invest. 124, 4823-4828 (2014).

59. Means, C. K. \& Brown, J. H. Sphingosine-1-phosphate receptor signalling in the heart. Cardiovasc. Res. 82, 193-200 (2009).

60. Ohuchi, H. et al. Expression patterns of the lysophospholipid receptor genes during mouse early development. Dev. Dyn. 237, 3280-3294 (2008).

61. Means, C. K., Miyamoto, S., Chun, J. \& Brown, J. H. S1P1 receptor localization confers selectivity for Gi-mediated cAMP and contractile responses. J. Biol. Chem. 283, 11954-11963 (2008).

62. Okusa, M. D. \& Lynch, K. R. Targeting sphingosine 1 phosphate receptor type 1 receptors in acute kidney injury. Drug Discov. Today Dis. Mech. 4, 55-59 (2007).

63. Lerman, L. O. \& Chade, A. R. Angiogenesis in the kidney: a new therapeutic target? Curr. Opin. Nephrol. Hypertens. 18, 160-165 (2009). 
64. Goligorsky, M. S., Kuo, M., Patschan, D. \& Verhaar, M. C. Review article: Endothelial progenitor cells in renal disease. Nephrology (Carlton, Vic.) 14, 291-297 (2009).

65. Dvorak, H. F., Brown, L. F., Detmar, M. \& Dvorak, A. M. Vascular permeability factor/vascular endothelial growth factor, microvascular hyperpermeability, and angiogenesis. Am. J. Pathol. 146, 1029-1039 (1995).

66. Gerwins, P., Sköldenberg, E. \& Claesson-Welsh, L. Function of fibroblast growth factors and vascular endothelial growth factors and their receptors in angiogenesis. Crit. Rev. Oncol. 34, 185-194 (2000).

67. Ham, A. et al. Selective deletion of the endothelial sphingosine-1-phosphate 1 receptor exacerbates kidney ischemia-reperfusion injury. Kidney Int. 85, 807-823 (2014).

68. Mozaffarian, D. et al. Heart disease and stroke statistics--2015 update: a report from the American Heart Association. Circulation 131, e29-322 (2015).

69. Epstein, J. A. Cardiac Development and Implications for Heart Disease. N. Engl. J. Med. 363, 1638-1647 (2010).

70. Moorman, A., Webb, S., Brown, N. A., Lamers, W. \& Anderson, R. H. Development of the heart: (1) formation of the cardiac chambers and arterial trunks. Heart 89, 806-814 (2003). 
71. Gessert, S. \& Kuhl, M. The multiple phases and faces of wnt signaling during cardiac differentiation and development. Circ. Res. 107, 186-199 (2010).

72. Souders, C. A., Bowers, S. L. \& Baudino, T. A. Cardiac fibroblast: the renaissance cell. Circ. Res. 105, 1164-1176 (2009).

73. Cai, C. L. et al. Isl1 identifies a cardiac progenitor population that proliferates prior to differentiation and contributes a majority of cells to the heart. Dev. Cell. 5, 877-889 (2003).

74. Buckingham, M., Meilhac, S. \& Zaffran, S. Building the mammalian heart from two sources of myocardial cells. Nat. Rev. Genet. 6, 826-835 (2005).

75. Bruneau, B. G. Signaling and Transcriptional Networks in Heart Development and Regeneration. Cold Spring Harbor Perspectives in Biology 5 (2013).

76. Harris IS, B. B. Development of the Endocardium. Pediatric Cardiology 31, 3, 391$399(2010)$.

77. Jain, R. et al. HEART DEVELOPMENT. Integration of Bmp and Wnt signaling by Hopx specifies commitment of cardiomyoblasts. Science 348, aaa6071 (2015).

78. Hirakow, R. Development of the cardiac blood vessels in staged human embryos. Acta Anat. (Basel) 115, 220-230 (1983).

79. Lyons, I. et al. Myogenic and morphogenetic defects in the heart tubes of murine embryos lacking the homeo box gene Nkx2-5. Genes Dev. 9, 1654-1666 (1995). 
80. Prall, O. W. et al. An Nkx2-5/Bmp2/Smad1 negative feedback loop controls heart progenitor specification and proliferation. Cell 128, 947-959 (2007).

81. Zhao, R. et al. Loss of both GATA4 and GATA6 blocks cardiac myocyte differentiation and results in acardia in mice. Dev. Biol. 317, 614-619 (2008).

82. Firulli, A. B., McFadden, D. G., Lin, Q., Srivastava, D. \& Olson, E. N. Heart and extraembryonic mesodermal defects in mouse embryos lacking the bHLH transcription factor Hand1. Nat. Genet. 18, 266-270 (1998).

83. Srivastava, D. et al. Regulation of cardiac mesodermal and neural crest development by the bHLH transcription factor, dHAND. Nat. Genet. 16, 154-160 (1997).

84. Bruneau, B. G. et al. A murine model of Holt-Oram syndrome defines roles of the T-box transcription factor Tbx5 in cardiogenesis and disease. Cell 106, 709-721 (2001).

85. Hu, T. et al. Tbx1 regulates fibroblast growth factors in the anterior heart field through a reinforcing autoregulatory loop involving forkhead transcription factors. Development 131, 5491-5502 (2004).

86. Xu, H. et al. Tbx1 has a dual role in the morphogenesis of the cardiac outflow tract. Development 131, 3217-3227 (2004).

87. Waldo, K. L. et al. Conotruncal myocardium arises from a secondary heart field. Development 128, 3179-3188 (2001). 
88. Abu-Issa, R., Smyth, G., Smoak, I., Yamamura, K. \& Meyers, E. N. Fgf8 is required for pharyngeal arch and cardiovascular development in the mouse. Development 129, 4613-4625 (2002).

89. Lin, Q., Schwarz, J., Bucana, C. \& Olson, E. N. Control of mouse cardiac morphogenesis and myogenesis by transcription factor MEF2C. Science 276, 14041407 (1997).

90. Takeuchi, J. K. et al. Tbx20 dose-dependently regulates transcription factor networks required for mouse heart and motoneuron development. Development 132, 2463-2474 (2005).

91. von Both, I. et al. Foxh1 is essential for development of the anterior heart field. Dev. Cell. 7, 331-345 (2004).

92. Sabin, F. R. in In: Contrib Embryol 61-124, 1917).

93. Lancrin, C. et al. Blood cell generation from the hemangioblast. J. Mol. Med. (Berl) 88, 167-172 (2010).

94. Liu, F. et al. Enhanced hemangioblast generation and improved vascular repair and regeneration from embryonic stem cells by defined transcription factors. Stem Cell. Reports 1, 166-182 (2013).

95. Kennedy, M. et al. A common precursor for primitive erythropoiesis and definitive haematopoiesis. Nature 386, 488-493 (1997). 
96. Zambidis, E. T. et al. Expression of angiotensin-converting enzyme (CD143) identifies and regulates primitive hemangioblasts derived from human pluripotent stem cells. Blood 112, 3601-3614 (2008).

97. Tavian, M., Biasch, K., Sinka, L., Vallet, J. \& Peault, B. Embryonic origin of human hematopoiesis. Int. J. Dev. Biol. 54, 1061-1065 (2010).

98. Nishikawa, S. Hemangioblast: an in vitro phantom. Wiley Interdisciplinary Reviews: Developmental Biology 1, 603-608 (2012).

99. Gothert, J. R. et al. Genetically tagging endothelial cells in vivo: bone marrowderived cells do not contribute to tumor endothelium. Blood 104, 1769-1777 (2004).

100. Gothert, J. R. et al. In vivo fate-tracing studies using the Scl stem cell enhancer: embryonic hematopoietic stem cells significantly contribute to adult hematopoiesis. Blood 105, 2724-2732 (2005).

101. Sequeira Lopez, M. L., Pentz, E. S., Robert, B., Abrahamson, D. R. \& Gomez, R. A. Embryonic origin and lineage of juxtaglomerular cells. Am. J. Physiol. Renal Physiol. 281, F345-56 (2001).

102. de Bruijn, M. F., Speck, N. A., Peeters, M. C. \& Dzierzak, E. Definitive hematopoietic stem cells first develop within the major arterial regions of the mouse embryo. EMBO J. 19, 2465-2474 (2000). 
103. Zovein, A. C. et al. Fate tracing reveals the endothelial origin of hematopoietic stem cells. Cell. Stem Cell. 3, 625-636 (2008).

104. Hasegawa, T. et al. The embryonic human choriocapillaris develops by hemovasculogenesis. Developmental Dynamics 236, 2089-2100 (2007).

105. Hyink, D. P. et al. Endogenous origin of glomerular endothelial and mesangial cells in grafts of embryonic kidneys. Am. J. Physiol. 270, F886-99 (1996).

106. Loughna, S. et al. A molecular and genetic analysis of renalglomerular capillary development. Angiogenesis 1, 84-101 (1997).

107. Kikkawa, Y., Virtanen, I. \& Miner, J. H. Mesangial cells organize the glomerular capillaries by adhering to the $\mathrm{G}$ domain of laminin alpha5 in the glomerular basement membrane. J. Cell Biol. 161, 187-196 (2003).

108. Lindahl, P. et al. Paracrine PDGF-B/PDGF-Rbeta signaling controls mesangial cell development in kidney glomeruli. Development 125, 3313-3322 (1998).

109. Vaughan, M. R. \& Quaggin, S. E. How do mesangial and endothelial cells form the glomerular tuft? J. Am. Soc. Nephrol. 19, 24-33 (2008).

110. Takemoto, M. et al. Large-scale identification of genes implicated in kidney glomerulus development and function. EMBO J. 25, 1160-1174 (2006).

111. Quaggin, S. E. et al. The basic-helix-loop-helix protein pod1 is critically important for kidney and lung organogenesis. Development 126, 5771-5783 (1999). 
112. Dreyer, S. D. et al. Mutations in LMX1B cause abnormal skeletal patterning and renal dysplasia in nail patella syndrome. Nat. Genet. 19, 47-50 (1998).

113. Chen, H. et al. Limb and kidney defects in Lmx1b mutant mice suggest an involvement of LMX1B in human nail patella syndrome. Nat. Genet. 19, 51-55 (1998).

114. Means, C. K. \& Brown, J. H. Sphingosine-1-phosphate receptor signalling in the heart. Cardiovasc. Res. 82, 193-200 (2009).

115. Zhang, J. et al. Signals from type 1 sphingosine 1-phosphate receptors enhance adult mouse cardiac myocyte survival during hypoxia. Am. J. Physiol. Heart Circ. Physiol. 293, H3150-8 (2007).

116. Means, C. K. et al. Sphingosine 1-phosphate S1P2 and S1P3 receptor-mediated Akt activation protects against in vivo myocardial ischemia-reperfusion injury. Am. J. Physiol. Heart Circ. Physiol. 292, H2944-51 (2007).

117. Srinivasan, R. S. et al. Lineage tracing demonstrates the venous origin of the mammalian lymphatic vasculature. Genes Dev. 21, 2422-2432 (2007).

118. Buttler, K. et al. Mesenchymal cells with leukocyte and lymphendothelial characteristics in murine embryos. Dev. Dyn. 235, 1554-1562 (2006).

119. Sebzda, E. et al. Syk and Slp-76 mutant mice reveal a cell-autonomous hematopoietic cell contribution to vascular development. Dev. Cell. 11, 349-361 (2006). 
120. Heinrich, A. C., Pelanda, R. \& Klingmuller, U. A mouse model for visualization and conditional mutations in the erythroid lineage. Blood 104, 659-666 (2004).

121. Yu, J., Carroll, T. J. \& McMahon, A. P. Sonic hedgehog regulates proliferation and differentiation of mesenchymal cells in the mouse metanephric kidney. Development 129, 5301-5312 (2002).

122. Soriano, P. Generalized lacZ expression with the ROSA26 Cre reporter strain. Nat. Genet. 21, 70-71 (1999).

123. Muzumdar, M. D., Tasic, B., Miyamichi, K., Li, L. \& Luo, L. A global doublefluorescent Cre reporter mouse. Genesis 45, 593-605 (2007).

124. Madisen, L. et al. A robust and high-throughput Cre reporting and characterization system for the whole mouse brain. Nat. Neurosci. 13, 133-140 (2010).

125. Wu, S., Wu, Y. \& Capecchi, M. R. Motoneurons and oligodendrocytes are sequentially generated from neural stem cells but do not appear to share common lineage-restricted progenitors in vivo. Development 133, 581-590 (2006).

126. Lakso, M. et al. Efficient in vivo manipulation of mouse genomic sequences at the zygote stage. Proc. Natl. Acad. Sci. U. S. A. 93, 5860-5865 (1996).

127. Sequeira Lopez, M. L., Pentz, E. S., Nomasa, T., Smithies, O. \& Gomez, R. A. Renin cells are precursors for multiple cell types that switch to the renin phenotype when homeostasis is threatened. Dev. Cell. 6, 719-728 (2004). 
128. Moretti, A. et al. Multipotent embryonic isl1+ progenitor cells lead to cardiac, smooth muscle, and endothelial cell diversification. Cell 127, 1151-1165 (2006).

129. Milgrom-Hoffman, M. et al. The heart endocardium is derived from vascular endothelial progenitors. Development 138, 4777-4787 (2011).

130. Katz, T. C. et al. Distinct compartments of the proepicardial organ give rise to coronary vascular endothelial cells. Dev. Cell. 22, 639-650 (2012).

131. Wu, B. et al. Endocardial cells form the coronary arteries by angiogenesis through myocardial-endocardial VEGF signaling. Cell 151, 1083-1096 (2012).

132. Ratajska, A., Czarnowska, E., Kolodzinska, A., Kluzek, W. \& Lesniak, W. Vasculogenesis of the embryonic heart: origin of blood island-like structures. Anat. Rec. A. Discov. Mol. Cell. Evol. Biol. 288, 223-232 (2006).

133. Morabito, C. J., Kattan, J. \& Bristow, J. Mechanisms of embryonic coronary artery development. Curr. Opin. Cardiol. 17, 235-241 (2002).

134. Bussmann, J., Bakkers, J. \& Schulte-Merker, S. Early endocardial morphogenesis requires Scl/Tal1. PLoS Genet. 3, e140 (2007).

135. Van Handel, B. et al. Scl represses cardiomyogenesis in prospective hemogenic endothelium and endocardium. Cell 150, 590-605 (2012). 
136. Hu, Y., Li, M., Gothert, J. R., Gomez, R. A. \& Sequeira-Lopez, M. L. Hemovascular Progenitors in the Kidney Require Sphingosine-1-Phosphate Receptor 1 for Vascular Development. J. Am. Soc. Nephrol. (2015).

137. Poulsen, R. R., McClaskey, C. M., Rivkees, S. A. \& Wendler, C. C. The Sphingosine1-phospate receptor 1 mediates S1P action during cardiac development. BMC Dev. Biol. 11, 37-213X-11-37 (2011).

138. Ohuchi, H. et al. Expression patterns of the lysophospholipid receptor genes during mouse early development. Developmental Dynamics 237, 3280-3294 (2008).

139. Red-Horse, K., Ueno, H., Weissman, I. L. \& Krasnow, M. A. Coronary arteries form by developmental reprogramming of venous cells. Nature 464, 549-553 (2010).

140. Kouskoff, V., Lacaud, G., Schwantz, S., Fehling, H. J. \& Keller, G. Sequential development of hematopoietic and cardiac mesoderm during embryonic stem cell differentiation. Proc. Natl. Acad. Sci. U. S. A. 102, 13170-13175 (2005).

141. Ema, M., Takahashi, S. \& Rossant, J. Deletion of the selection cassette, but not cis-acting elements, in targeted Flk1-lacZ allele reveals Flk1 expression in multipotent mesodermal progenitors. Blood 107, 111-117 (2005).

142. Misfeldt, A. M. et al. Endocardial cells are a distinct endothelial lineage derived from Flk1+ multipotent cardiovascular progenitors. Dev. Biol. 333, 78-89 (2009). 
143. Okuda, T., van Deursen, J., Hiebert, S. W., Grosveld, G. \& Downing, J. R. AML1, the target of multiple chromosomal translocations in human leukemia, is essential for normal fetal liver hematopoiesis. Cell 84, 321-330 (1996).

144. Hirschi, K. K. Hemogenic endothelium during development and beyond. Blood 119, 4823-4827 (2012).

145. Weiford, B. C., Subbarao, V. D. \& Mulhern, K. M. Noncompaction of the Ventricular Myocardium. Circulation 109, 2965-2971 (2004).

146. Srivastava, D. \& Olson, E. N. A genetic blueprint for cardiac development. Nature 407, 221-226 (2000).

147. Lee, K. F. et al. Requirement for neuregulin receptor erbB2 in neural and cardiac development. Nature 378, 394-398 (1995).

148. Tian, X., Pu, W. T. \& Zhou, B. Cellular origin and developmental program of coronary angiogenesis. Circ. Res. 116, 515-530 (2015).

149. Chen, H. I. et al. The sinus venosus contributes to coronary vasculature through VEGFC-stimulated angiogenesis. Development 141, 4500-4512 (2014).

150. Sariola, H., Ekblom, P., Lehtonen, E. \& Saxen, L. Differentiation and vascularization of the metanephric kidney grafted on the chorioallantoic membrane. Dev. Biol. 96, 427-435 (1983). 
151. Ramírez-Bergeron, D. L. et al. Hypoxia affects mesoderm and enhances hemangioblast specification during early development. Development 131, 46234634 (2004).

152. Fantin, A. et al. Tissue macrophages act as cellular chaperones for vascular anastomosis downstream of VEGF-mediated endothelial tip cell induction. Blood 116, 829-840 (2010).

153. Krishnan, J. et al. Essential role of developmentally activated hypoxia-inducible factor 1alpha for cardiac morphogenesis and function. Circ. Res. 103, 1139-1146 (2008).

154. Lavine, K. J. \& Ornitz, D. M. Shared circuitry: developmental signaling cascades regulate both embryonic and adult coronary vasculature. Circ. Res. 104, 159-169 (2009).

155. Lluri, G. \& Aboulhosn, J. Coronary arterial development: a review of normal and congenitally anomalous patterns. Clin. Cardiol. 37, 126-130 (2014).

156. McMahon, A. P. et al. GUDMAP: the genitourinary developmental molecular anatomy project. J. Am. Soc. Nephrol. 19, 667-671 (2008).

157. Harding, S. D. et al. The GUDMAP database--an online resource for genitourinary research. Development 138, 2845-2853 (2011).

158. Paik, J. H. et al. Sphingosine 1-phosphate receptor regulation of $\mathrm{N}$-cadherin mediates vascular stabilization. Genes Dev. 18, 2392-2403 (2004). 
159. Notoya, M., Shinosaki, T., Kobayashi, T., Sakai, T. \& Kurihara, H. Intussusceptive capillary growth is required for glomerular repair in rat Thy-1.1 nephritis. Kidney Int. 63, 1365-1373 (2003).

160. Zamboni, L. \& Martino, C. Embryogenesis of the human renal glomerulus. I. A histologic study. <br />. Arch. Pathol. 86, 279-291 (1968).

161. Osathanondh, V. \& Potter, E. L. Development of human kidney as shown by microdissection. V. Development of vascular pattern of glomerulus. Arch. Pathol. 82, 403-411 (1966).

162. Schell, C., Wanner, N. \& Huber, T. B. Glomerular development--shaping the multi-cellular filtration unit. Semin. Cell Dev. Biol. 36, 39-49 (2014).

163. Niessen, K. et al. The Notch1-Dll4 signaling pathway regulates mouse postnatal lymphatic development. Blood 118, 1989-1997 (2011).

164. Wang, Y. \& Simons, M. Flow-regulated lymphatic vasculature development and signaling. Vasc. Cell. 6, 14-824X-6-14. eCollection 2014 (2014).

165. Yan, Z. X., Jiang, Z. H. \& Liu, N. F. Angiopoietin-2 promotes inflammatory lymphangiogenesis and its effect can be blocked by the specific inhibitor L1-10. Am. J. Physiol. Heart Circ. Physiol. 302, H215-23 (2012).

166. Jang, C. et al. Angiopoietin-2 exocytosis is stimulated by sphingosine-1phosphate in human blood and lymphatic endothelial cells. Arterioscler. Thromb. Vasc. Biol. 29, 401-407 (2009). 
167. Chae, S. S., Paik, J. H., Allende, M. L., Proia, R. L. \& Hla, T. Regulation of limb development by the sphingosine 1-phosphate receptor S1p1/EDG-1 occurs via the hypoxia/VEGF axis. Dev. Biol. 268, 441-447 (2004).

168. Zampell, J. C. et al. HIF- $1 \alpha$ coordinates lymphangiogenesis during wound healing and in response to inflammation. The FASEB Journal 26, 1027-1039 (2011).

169. Liao, S., Padera, T. P. \& Jain, R. K. Notch leads lymphatics and links them to blood vessels. Arterioscler. Thromb. Vasc. Biol. 30, 1682-1683 (2010).

170. Geudens, I. et al. Role of delta-like-4/Notch in the formation and wiring of the lymphatic network in zebrafish. Arterioscler. Thromb. Vasc. Biol. 30, 1695-1702 (2010).

171. Dunlap, K. A. et al. The sphingosine 1-phosphate (S1P) signaling pathway is regulated during pregnancy in sheep. Biol. Reprod. 82, 876-887 (2010). 